\title{
NFIC regulates ribosomal biology and ER stress in pancreatic acinar cells and suppresses PDAC initiation
}

\author{
Isidoro Cobo, ${ }^{1,2}$ Sumit Paliwal, ${ }^{1}$ Júlia Melià-Alomà, ${ }^{1,3}$ Ariadna Torres, ${ }^{1,3}$ \\ Jaime Martínez-Villarreal, ${ }^{1}$ Fernando García, ${ }^{4}$ Irene Millán, ${ }^{1,2}$ Natalia del Pozo, ${ }^{1,2}$ Joo- \\ Cheol Park, ${ }^{5}$ Ray J. MacDonald, ${ }^{6}$ Javier Muñoz, ${ }^{4}$ and Francisco X. Real ${ }^{1-3}$ \\ Short title: NFIC in pancreatic homeostasis and cancer \\ ${ }^{1}$ Epithelial Carcinogenesis Group, Spanish National Cancer Research Centre-CNIO, \\ Madrid, Spain
}

${ }^{2}$ CIBERONC, Madrid, Spain

${ }^{3}$ Departament de Ciències Experimentals i de la Salut, Universitat Pompeu Fabra, Barcelona, Spain

${ }^{4}$ Proteomics Unit, Spanish National Cancer Research Centre-CNIO, Madrid, Spain. ProteoRed - ISCIII

${ }^{5}$ Department of Oral Histology-Developmental Biology, School of Dentistry, Seoul National University, Seoul, Korea

${ }^{6}$ Department of Molecular Biology, University of Texas Southwestern Medical Center, Dallas, TX, USA

Correspondence: Francisco X. Real Centro Nacional de Investigaciones Oncológicas-CNIO Melchor Fernández Almagro, 3 28029-Madrid, Spain E-mail: preal@cnio.es

Conflicts of interest: none to declare

Funding: This work was supported, in part, by grants SAF2011-29530, SAF2015-70553R, and RTI2018-101071-B-I00 from Ministerio de Ciencia, Innovación y Universidades (Madrid, Spain) (co-funded by the ERDF-EU) and RTICC from Instituto de Salud Carlos III (RD12/0036/0034) to FXR. IC was recipient of a Beca de Formación del Personal Investigador from Ministerio de Economía y Competitividad (Madrid, Spain). The research leading to these results has received funding from People Programme (Marie 
42 Curie Actions) of the European Union's Seventh Framework Programme (FP7/2007-

43 2013) (REA grant agreement $n^{\circ} 608765$ "). SP was supported by a Juan de la Cierva

44 Programme fellowship from Ministerio de Ciencia, Innovación y Universidades. IM was

45 supported by a Fellowship from Fundació Bancaria La Caixa (ID 100010434) (grant

46 number LCF/BQ/ES18/11670009). CNIO is supported by Ministerio de Ciencia,

47 Innovación y Universidades as a Centro de Excelencia Severo Ochoa SEV-2015-0510.

\section{Statement of author contributions}

50 IC: study concept and design; acquisition of data; analysis and interpretation of data; 51 statistical analysis; drafting of the manuscript;

52 SP: acquisition of data; analysis and interpretation of data; drafting of the manuscript

53 JMA: acquisition of data; analysis and interpretation of data; drafting of the manuscript

54 AT: acquisition of data; analysis and interpretation of data;

55 JMV: analysis and interpretation of data;

FG: acquisition of data; analysis and interpretation of data;

57 IM: analysis and interpretation of data;

NdP: technical support and acquisition of data;

JCP: material support;

60 RJM: critical revision of the data and important intellectual content;

$61 \mathrm{JM}$ : acquisition of data; analysis and interpretation of data;

62 FXR: study concept and design; analysis and interpretation of data; drafting of the manuscript; overall study supervision; obtained funding.

64 All authors provided input about manuscript content.

65 Accession numbers: RNA sequencing data have been deposited in GEO with 66 accession number GSE126907 and NFIC ChIP sequencing data have been deposited 67 in GEO with accession number GSE181098 


\section{ABSTRACT}

70

Tissue-specific differentiation is driven by specialized transcriptional networks. Pancreatic acinar cells crucially rely on the PTF1 complex, and on additional transcription factors, to deploy their transcriptional program. Here, we identify NFIC as a novel regulator of acinar differentiation using a variety of methodological strategies. NFIC binding sites are found at very short distances from NR5A2-bound genomic regions and both proteins co-occur in the same complex. Nfic knockout mice show reduced expression of acinar genes and, in ChIP-seq experiments, NFIC binds the promoters of acinar genes. In addition, NFIC binds to the promoter of, and regulates, genes involved in RNA and protein metabolism; in Nfic knockout mice, p-RS6K1 and p-IEF4E are downregulated indicating reduced activity of the mTOR pathway. In 266-6 acinar cells, NFIC dampens the ER stress program through its binding to ER stress gene promoters and is required for complete resolution of Tunicamycin-mediated ER stress. Normal human pancreata from subjects with low NFIC mRNA levels display reduced epxression of genes down-regulated in Nfic knockout mice. Consistently, NFIC displays reduced expression upon induced acute pancreatitis and is required for proper recovery after damage. Finally, expression of NFIC is lower in samples of mouse and human pancreatic ductal adenocarcinoma and Nfic knockout mice develop an increased number of mutant Kras-driven pre-neoplastic lesions.

\section{Word count: 211}

Keywords: NFIC, pancreas, acinar differentiation, ribosome, endoplasmic reticulum stress, unfolded protein response, transcriptional networks, pancreatitis, pancreatic cancer

Abbreviations: ChIP, chromatin immunoprecipitation; DEG, differentially expressed genes; EMT, epithelial-mesenchymal transition; ER, endoplasmic reticulum; GSEA, Gene set enrichment analysis; IF, immunofluorescence; IHC; immunohistochemistry; PDAC, pancreatic ductal adenocarcinoma; TF, transcription factor; TM, tunicamycin; UPR, unfolded protein response. 
Pancreatic acinar cells are highly specialized protein synthesis factories that have a well-developed rough endoplasmic reticulum (ER), a prominent Golgi complex, and abundant secretory granules ${ }^{1}$. Acinar differentiation is contingent on the activity of a master regulator, the adult PTF1 complex, composed of the pancreas-specific transcription factors (TFs) PTF1A and RPBJL and the ubiquitous protein E47 ${ }^{2,3}$. PTF1

109 binds the proximal promoter of genes coding for digestive enzymes, secretory proteins 110 and other TFs, and activates their expression. The PTF1 complex is the main driver of 111 acinar differentiation but additional TF with tissue-restricted expression patterns are 112 implicated in the fine-tuning of this process, including GATA6 ${ }^{4}, \mathrm{MIST}^{5}$, and

$113 \mathrm{NR} 5 \mathrm{~A} 2 / \mathrm{LRH}-1^{6,7}$. Acinar cells play a crucial role in acute and chronic pancreatitis, two 114 common and disabling conditions. Recent work using genetic mouse models has shown 115 that, upon expression of mutant KRas, acinar cells can be the precursors of Pancreatic Intraepithelial Neoplasia (PanIN) and pancreatic ductal adenocarcinoma (PDAC) $)^{8,9}$.

Our laboratory and others have shown that the acinar differentiation program acts 118 as a tumor suppressor in the pancreas. Monoallelic or homozygous inactivation of 119 several acinar transcriptional regulators in the germline, the embryonic pancreas, or the 120 adult pancreas can result in compromised acinar function that favors loss of cellular 121 identity and poises acinar cells for transformation upon activation of mutant KRas ${ }^{10,11,12}$.

122 The tumor suppressive function of these TF is not obvious because the exocrine 123 pancreas has a large functional reserve, i.e. massive alterations in cellular function need 124 to occur in order to be reflected in histological or clinical changes.

125 Here, we use bioinformatics tools to identify NFIC as a novel acinar regulator.

126 NFIC is a member of the nuclear factor I family of TFs that regulate both ubiquitous and 127 tissue-restricted genes ${ }^{13}$. In the mammary gland, NFIC activates the expression of milk 128 genes involved in lactation ${ }^{14}$. Furthermore, it acts as a breast cancer tumor suppressor, 129 as it directly represses the expression of Ccnd1 and Foxf1, a potent inducer of epithelial130 mesenchymal transition (EMT), invasiveness, and tumorigenicity. Additional roles have 131 been proposed through the regulation of Trp5315,16,17. The physiological role of NFIC has 132 been best studied in dentinogenesis, since $\mathrm{Nfic}^{-/}$mice develop short molar roots and 133 display aberrant odontoblast differentiation and dentin formation ${ }^{18}$. NFIC regulates odontoblast-related genes, including $D s s p^{19}, \mathrm{Wnt}^{20}$, and hedgehog signaling ${ }^{21}$. 
136 cells, we now uncover novel roles of NFIC as a regulator of acinar function whose major

137 impact is at the level of the ER stress response in murine and human pancreas. Unlike

138 most other TFs previously identified as required for full acinar function, NFIC belongs to

139 a novel family of acinar regulators with tissue-wide expression. NFIC dysregulation

140 sensitizes the pancreas to damage and neoplastic transformation.

141

142 


\section{RESULTS}

144 Identification of novel transcription factors involved in the regulation of pancreatic

145 acinar differentiation. To discover novel transcription factors that might cooperate with 146 known acinar regulators (e.g. PTF1A, GATA6, NR5A2, and MIST1), we reanalyzed 147 publicly available ChIP-sequencing data and used HOMER to search for motifs enriched 148 in the sequencing reads. As expected, the cognate binding sites of these factors were 149 the top enriched motif in each respective analysis (Figure $1 \mathrm{~A}$ ). Motifs corresponding to 150 RBPJ/RBPJL and HNF1, known regulators of acinar differentiation, were also enriched, 151 thus validating the strategy applied. In addition, we found consistent enrichment of the $152 \mathrm{NF1}$ (CTF)/NFIC motif across all the experiments, with lowest p-values in the NR5A2 153 ChIP-Seq dataset. This motif is significantly enriched in NR5A2 ChIP-Seq peaks from 154 normal adult mouse pancreas ${ }^{6}$ (Figure $1 \mathrm{~A}$ ) but not in those from E17.5 pancreas ${ }^{7}$ nor 155 from mouse ES cells ${ }^{22}$, pointing to temporal and lineage identity specificity (Figure 1B).

156 Of all NFI family members, NFIC is expressed at highest levels in both mouse and human 157 pancreas (Figure 1C); therefore, we focused on NFIC for further study. Using 158 immunoprecipitation and western blotting, we found that NFIC and NR5A2 are present 159 in the same complex in normal adult pancreas - but not in E17.5 pancreas (Figure 1D). 160 Analysis of the spacing between NR5A2 and NFIC motifs in the genomic regions bound 161 by NR5A2, using the SpaMo tool from MEME suite, showed that NFIC binding motifs are 162 located in close proximity to NR5A2 binding motifs, with a spacing of 29 nucleotides 163 being the most significantly conserved distance $(P=e-16)$ (Figure 1E). Analysis of the 164 published ChIP-Seq data revealed several PTF1A and NR5A2 peaks in the proximal Nfic 165 promoter and the binding was confirmed by ChIP-qPCR (Figure 1F), strongly suggesting 166 that Nfic is a PTF1A and NR5A2 target. Using ChIP-qPCR, NFIC was found to bind the 167 promoter of bona fide acinar genes such as Cela2a, Cpa1, Ctrb1, Pnlip and Nrob2 that 168 were similarly bound by NR5A2 (Figure 1G). The above observations support the notion 169 that NFIC is a novel pancreatic acinar transcription factor network member.

170 To assess the cellular distribution of NFIC, we performed immunohistochemistry 171 (IHC) with a well-validated antibody. In normal 8 week-old pancreas, NFIC is expressed 172 at high levels in acinar cells and at lower levels in endocrine and ductal cells (Figure $1 \mathrm{H}$ ). 173 These results were validated using triple immunofluorescence (IF) with antibodies 174 detecting PTF1A, INS1, and KRT19 (Supplementary Figure 1). In contrast, NFIC was 175 undetectable at $\mathrm{E} 12.5$ and $\mathrm{E} 14.5$ in $\mathrm{CDH1}^{+} ; \mathrm{PTF} \mathrm{A}^{+}$pancreatic progenitors 176 (Supplementary Figure 2A,B) but it was detected at E16.5 and E18.5 in PTF1 $\mathrm{A}^{+}$acinar 177 as well as in KRT19- and INS1-expressing cells (Supplementary Figure 2C,D). 
To determine whether NFIC is required for the expression of digestive enzyme transcripts, we first knocked down Nfic in 266-6 acinar cells using lentiviral shRNAs: a

180 significant down-regulation of Ctrb1 and Cela2a - as well as Ptf1a and Rbpjl - was demonstrated. Expression of PTF1A, CTRB1, and CPA proteins was similarly reduced

182 (Figure $1 \mathrm{H}$ ), suggesting an important role for NFIC in the regulation of late stages of acinar differentiation.

NFIC is part of the transcriptional network responsible for acinar identity and function. To further assess the role of $\mathrm{Nfic}$ in pancreatic development and homeostasis, we used constitutive Nfic knockout mice ${ }^{18}$. Nfic/- mice are viable and have a normal weight at 8 weeks (not shown. $\mathrm{Nfic}^{-/}$pancreata appeared histologically normal and we did not find major differences in the expression of INS1, KRT19, and SOX9 (Supplementary Figure $3 \mathrm{~A}$ ), indicating that NFIC is not crucially required for pancreas development or differentiation. Results of glucose tolerance tests were similar in 8 weekold control and $\mathrm{Nfic}^{-/}$mice, except that glucose levels were reduced by $60-120 \mathrm{~min}$ in the latter (Supplementary Figure 3B,C).

Because histology lacks sensitivity to disclose subtle alterations in exocrine function ${ }^{4,10,23}$ we performed RNA-Seq of pancreata from 8-10 week-old wild type and $\mathrm{Nfic}^{/-}$mice. We identified 1641 and 1568 transcripts that were significantly up- and downregulated, respectively, in $\mathrm{Nfic}^{-}$pancreata. Multiple genes belonging to the exocrine differentiation program were among the down-regulated transcripts (e.g. Ptf1a, several digestive enzymes, and the acinar-specific kinase $M k n k 1^{24}$ and the differences were confirmed using qRT-PCR (Figure 2A) and at the protein level (Figure 2B,C, Supplementary Figure 4A,B). The RNA-Seq data also showed reduced expression of genes involved in epithelial polarity (e.g. Muc1) and cell adhesion (e.g. Cdh1) and upregulation of transcripts coding for EMT markers (e.g. Vimentin, Twist, and Ncadherin/Cdh2; not shown). The down-regulation of Cdh1 is in accordance with findings in dentinogenesis ${ }^{19}$. NR5A2 expression was similar in control and $\mathrm{Nfic}^{-/}$pancreata

206 (Figure 2B), indicating that the effects of Nfic inactivation are not secondary to changes in NR5A2 expression.

Up-regulated transcripts were enriched in inflammatory/immune gene sets, including chemokines (e.g. S100a8, S100a9, Ccl5, Ccl7, Cxcl12, Cxcl3) and

210 complement components (e.g. C1qb, C3, Cfb, Cfd) (Supplementary Figure 5A). Selected

211 changes were validated by RT-qPCR in total pancreas and in freshly isolated acini

212 (Supplementary Figure 5B). These genes have putative NFI binding sites in their 213 promoter region [-950bp; +50bp] (Supplementary Figure 5C). The up-regulation of 214 inflammatory gene transcripts was accompanied by a 2 -fold increase of CD45+ cells and 
$215 \mathrm{Ki}^{6} 7^{+}$acinar cells (Supplementary Figure 5D-F). These results suggest that NFIC

216 contributes to restrain an inflammatory program in the pancreas.

217 We quantified the overlap of differentially expressed genes in $\mathrm{Nfic}^{-/}$pancreata 218 with that in mice in which $N r 5 a 2^{6}, P t f 1 a^{25}$ or Mist ${ }^{26}$ has been inactivated in the pancreas 219 (PKO). We found a significant overlap of genes down-regulated in the pancreas of Nfic 220 /- and Nr5a2 pancreas-knockout, Ptf1a pancreas- knockout, or Mist1 knockout mice [41\% 221 (52/126), 46.97\% (231/492), 57.08\% (262/459), respectively] but not of the up-regulated 222 genes [2.36\% (3/127), 9.99\% (41/414), 16.37\% (75/458), respectively] (Figure 2D)

223 These findings strongly suggest that NFIC is a novel member of the acinar transcription 224 factor network.

225 To identify direct NFIC target genes we performed ChIP-seq using pancreata 226 from 8-10 week-old wild type mice. A total of 15824 peaks bound by NFIC were identified, 227 corresponding to 9086 genes, with enrichment of motifs corresponding to NF1, ATOH1, 228 and TF involved in acinar cell differentiation such as FOXA1, GATA6, and nuclear 229 receptors, among others (Figure $3 A$ ). NFIC peaks were enriched in the vicinity of the 230 TSS of genes [-1000; TSS, 22.66\%] and these peaks displayed significant enrichment of NF1, SP2, THAP, ELK1 and AP-1 motifs (Figure 3B).

RNA-seq and ChIP-seq data were integrated to unveil genes/pathways directly 233 regulated by NFIC: $36.3 \%(593 / 1634)$ of the differentially up-regulated genes $(P<0.05)$ 234 and $55.54 \%(871 / 1568)$ of the down-regulated genes in Nfic-/- pancreata were bound by 235 NFIC $(P<0.05)$. A greater percentage of down-regulated genes $(47.19 \%, 411 / 871)$ 236 relative to up-regulated genes $(36.42 \%, 216 / 593)$, had NFIC peaks in the putative 237 promoter region [-1000:TSS] (Figure 3C). The proportion of NFIC high affinity peaks as 238 defined by the top two quartiles of peak score $(\mathrm{Q} 1+\mathrm{Q} 2)$ was higher in down-regulated 239 genes relative to up-regulated genes (60\% vs. $51 \%$, respectively. $P<0.05)$ (Figure 3D).

240 The motifs enriched in down-regulated genes with NFIC peaks [-1000;TSS] include NFI, 241 FOXM1, bHLH, RBPJ, and ARID5A; those enriched in up-regulated genes included NFI, 242 TLX, HNF1, TCF3, and CTCF (Figure 3E and F, respectively). Gene set enrichment 243 analysis showed that NFIC-bound down-regulated genes were associated with acinar 244 differentiation, protein metabolism (e.g. oxidative phosphorylation, protein export, 245 ribosome, seleno amino acid and purine metabolism, among others). Exemplary genes 246 include Amy2a5, Bhlha15, Cel, Cela1, Cela2b, Cpa1, Nrob2, Pnliprp1, Rpl10a and 247 Rpl23a (Figure 3G). In contrast, NFIC-bound up-regulated genes were enriched in cell 248 adhesion and inflammatory pathways (e.g. chemokine signaling, leukocyte 249 transendothelial migration, ECM receptor interaction), MAPK signaling, and pathways in 250 cancer including inflammatory genes (Figure $3 \mathrm{H}$ ). Exemplary genes include Cfi, Fos, 251 genes involved in ER stress and UPR Dnajc5, Dnajc13, Hsp90aa1, circadian clock 
252 regulator Per2, and Pparg, Rara, and Rarg (Figure 3H). These pathways have been 253 shown to be critically relevant in pancreatic homeostasis and disease $23,27-32$. 254 Representative examples of ChIP-Seq findings are displayed in Figure 31.

NFIC distinctly regulates the ribosomal program and aspects of the unfolded protein and ER stress responses. A striking finding from the GSEA analysis was the enrichment in gene sets related to protein synthesis (Supplementary Figure 6A). Multiple transcripts coding for ribosomal proteins were down-regulated in $\mathrm{Nfic}^{-/}$ pancreata (Supplementary Figure 6B) and reactivity with an antibody detecting the 5.8S rRNA - a surrogate readout of ribosomes - was reduced in $\mathrm{Nfic}^{-}$- acinar cells (Figure $4 A, B)$. In addition, there was a down-regulation of ER and Golgi complex components, including Fkbp2, Dio and Pink1 that were bound by NFIC at their promoter region

264 (Supplementary Figure 6C-E). The dysregulation of protein metabolism suggests a role of the mTOR pathway ${ }^{33}$. Western blotting analysis of $\mathrm{Nfic}^{-/}$pancreata showed reduced expression of phosphorylated ribosomal S6 Kinase 1 (P-RS6K1), its substrate S6 ribosomal protein (P-RS6), and phospho-elongation initiation factor 4e (P-EIF4E), together with a modest up-regulation of P-ERK (Figure 4C). IHC confirmed that these changes occur in acinar cells (Figure 4D-F). Interestingly, several ribosomal genes were found to be bound by NFIC in Chip-Seq experiments with a variety of cell types from the ENCODE project (Supplementary Figure 6F). These results indicate that NFIC loss impacts on ribosomal biogenesis and on the activity of the mTOR pathway in the adult mouse pancreas.

To determine whether similar findings apply to normal human pancreas, we used the GTEX dataset $(n=171)$ and compared gene expression in samples with high vs. low NFIC levels (top vs. bottom 10 individuals): there was a -2.06-fold log2 difference in NFIC transcript levels in in NFIClow vs. NFIChigh pancreata. Ninety-four percent of genes that were down-regulated in $\mathrm{Nfic}^{--}$pancreata were also down-regulated in $\mathrm{NFIC}$ low human pancreata $\left(P=1.79 \mathrm{e}^{-47}\right)$ compared to $63 \%$ of a random gene list (Figure $\left.4 \mathrm{G}\right)$. Among the common down-regulated genes are several involved in ribosomal function (Figure $4 \mathrm{H}$ ), including RPS5, RPS8, RPS11, RPS15, RPS21, RPS26, and RPS29 (Figure 4I). By contrast, only $27 \%$ of transcripts with up-regulated expression in $\mathrm{Nfic}^{-/}$pancreata were up-regulated in $N F I C^{\text {low }}$ human samples, compared to $37 \%$ of a random list of genes $\left(\mathrm{P}=3.47 \mathrm{e}^{-8}\right)$, including RPS5, RPS8, RPS11, RPS15, RPS21, RPS26, RPS29 (Figure $4 \mathrm{H}, \mathrm{I})$. These data support a conservation of the function of NFIC in normal pancreas in mice and humans.

A large number of NFIC-bound down-regulated genes are involved in autophagy (e.g. Ulk1, Prkaa2, Pik3c3, Gabarap, Gabarapl1, Map1lc3b, Sqstm1, Pink1, Dap). 
289 Alterations in the unfolded protein response (UPR) and autophagy induce ER stress ${ }^{34}$.

290 Accordingly, we found an up-regulation of transcripts of NFIC-bound genes coding for

291 ER stress proteins (Figure 5A, Supplementary Figure 7A). Up-regulation of Chop/Ddit3,

292 Hspa5/Bip-1, and spliced Xbp1 (sXbp1) mRNAs was confirmed using RT-qPCR (Figure

293 5B). We observed a modest, significant, up-regulation of BIP-1 and CHOP in Nfic ${ }^{-/}$

294 pancreata (Figure 5C,D); BIP-1 up-regulation was confirmed by IF (Supplementary

295 Figure 7B,C). In addition, we found up-regulation of UPR genes in the pancreas of

296 individuals with low NFIC including HSPA90AA1, CALR3, HSPA6 and, to a lower extent,

297 CHOP, LDLR and TSEN15 (Supplementary Figure 7D) Moreover, NFIC binds to the

298 proximal promoter or distal region of $28.31 \%$ (43/81) and $24.69 \%$ (20/81), respectively,

299 of the genes associated to ER stress (Supplementary Figure 7E) (e.g. Ddit4 and Slc1a5)

300 (Supplementary Figure 7F). Interestingly, NFIC - but not NR5A2 - bound to the promoter

301 of Hspa5/Bip-1, Ddit3/Chop, and Hsp90aa1 (Figure 5E), highlighting that NFIC

302 selectively regulates an aspect of the acinar secretory program related to the ER stress 303 response.

304 To determine whether NFIC is involved in the ER stress response in the 305 pancreas, 266-6 cells were treated with tunicamycin (TM), a protein N-glycosylation 306 inhibitor. We also analyzed NR5A2 since it has been shown to participate in this process 307 in the liver ${ }^{35}$. As expected, we observed a dose-dependent up-regulation of BIP-1 and a 308 down-regulation of NFIC, NR5A2, and P-S6 at 24h. By 36h, NFIC levels remained low 309 whereas NR5A2 expression had recovered (Supplementary Figure 7G). Nfic knock310 down in 266-6 cells did not affect basal BIP-1 or CHOP expression but it sensitized cells

311 to the effects of TM (Figure 5F,G). Accordingly, NFIC-overexpressing cells showed 312 reduced expression of BIP-1 and CHOP upon treatment with TM (Figure $5 \mathrm{H}, \mathrm{I})$. In both 313 cases, NR5A2 expression was unaffected (Figure 5F,H). Overall, these results indicate 314 that NFIC regulates multiple aspects of protein synthesis biology and the ER stress 315 response in pancreatic acinar cells. 
317 Nfic is required for recovery after induction of pancreatic damage. Acute

318 pancreatitis is associated with a down-regulation of TFs involved in acinar differentiation

319 and the up-regulation of ER stress and the UPR ${ }^{36,37}$. After induction of an acute caerulein

320 pancreatitis (7 hourly doses) in wild type mice, Nfic mRNA levels decreased at early time

321 points (8h) and were gradually restored upon recovery (Figure 6A). A similar expression

322 pattern was observed at the protein level (Figure 6B,C). To investigate whether Nfic

323 inactivation affects damage and/or regeneration, we induced a mild acute caerulein

324 pancreatitis in control and knockout mice. At early time points (1-24h), wild-type and Nfic

325 /- pancreata showed similar damage (Figure 6D). However, at $48 \mathrm{~h} \mathrm{~N} \mathrm{Nic}^{-1}$ pancreata

326 showed more prominent oedema, leukocyte infiltration, multifocal ADM, and acinar

327 vacuolization. These differences persisted up to day 5 (Figure 6D,E and Supplementary

328 Figure $7 \mathrm{H}$ ). IHC confirmed an increased number of $\mathrm{CD}_{4} 5^{+}, \mathrm{KRT}^{+} 9^{+}$, and $\mathrm{KI}^{2} 7^{+}$acinar

329 cells in $\mathrm{Nfic}^{--}$pancreata (Figure 6F,G). These changes were accompanied by up-

330 regulation of Ddit3/Chop and Hsp17b11 mRNA in Nfic/- pancreata at $48 \mathrm{~h}$ and day 5

331 (Figure $6 \mathrm{H}$ ). These results indicate that NFIC is required for the recovery from pancreatic

332 damage.

333

334 NFIC suppresses PDAC initiation. Pancreatitis sensitizes the pancreas to the 335 oncogenic effects of mutant Kras. We first analyzed NFIC expression in murine PanINs 336 and PDAC from Ptf1a-Cre ${ }^{+/ K I} ; K_{R a s G 12 V+/ K I}(\mathrm{KC})$ using IHC and found that it is down337 regulated in both preneoplastic and tumor cells (Figure 7A,B). Similar findings were 338 made in samples from patients: we found significant down-regulation of NFIC mRNA in 339 PDAC $(n=118)$ when compared to normal tissue $(n=13)$ (Figure $7 B)^{38}$. Using IHC, we

340 found that NFIC is consistently down-regulated in PanINs of low $(n=56)$ and high $(n=34)$ 341 grade and in a subset of PDAC samples $(n=43)$ (Figure 7C,D, right panel). Analysis of 342 the PanCuRx microdissected PDAC dataset showed that NFIC mRNA expression was 343 similar in classical- and basal-type tumors ( not shown).

344 TFs involved in acinar differentiation have been shown to suppress tumor

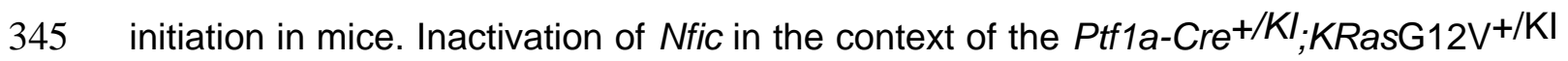

346 alleles resulted in an increased number of PanINs (and of the relative area ocupied by 347 them) in 18-24 week-old mice (Figure 7E,F). Altogether, these findings support a role of 348 NFIC in the suppression of PDAC initiation. 


\section{DISCUSSION}

352 NFIC is a novel regulator of the pancreatic acinar program. Acinar differentiation was

353 long thought to be a "digital" process controlled by PTF1. Increasing evidence supports

354 an "analog" differentiation model whereby additional TFs are required for "completion"

355 of this process. Among them are NR5A2 ${ }^{6,7}, \mathrm{HNF}^{2} \mathrm{~A}^{36}, \mathrm{GATA}^{4}, \mathrm{MIST} 1^{5,26}$, and XBP1 39.

356 Here, we show that NFIC, a ubiquitous TF, is a novel acinar regulator present in a

357 complex with NR5A2. While NFIC is not crucially required for pancreas organogenesis,

358 in adult mice it regulates the ribosomal program and ER stress response and is

359 dysregulated in pancreatitis and cancer.

360 We identified NFIC as an NR5A2 partner through co-binding in normal mouse

361 pancreas but it remains to be determined whether both proteins interact directly. The

362 conservation of spacing between NR5A2 and NFIC motifs among NR5A2 target genes

363 supports transcriptional cooperation. However, comparison of NR5A2 ChIP-Seq data

364 from embryonic and adult pancreas indicates that the role of NFIC is mainly in the latter,

365 supporting its requirement for completion of acinar maturation and highlighting a

366 functional role distinct from that of NR5A2. The transcriptional program driven by NFIC

367 overlaps partially with that of the tissue-restricted PTF1A, NR5A2, and MIST1 factors,

368 indicating that multiple TFs cooperate to activate acinar differentiation. However,

369 inactivation of $\mathrm{Nfic}$ has milder effects than inactivation of Ptf1a or Nr5a2, possibly

370 because the latter act at earlier stages of pancreatic development. NFI proteins were

371 first proposed to be involved in the regulation of ubiquitous genes but they can also

372 regulate tissue-specific genes ${ }^{13}$, including CEL in the mammary gland and DSPP in

373 odontoblasts ${ }^{14,19}$. We show that NFIC also regulates the acinar program in the pancreas.

374 The down-regulation of $\mathrm{CDH} 1 \mathrm{mRNA}$ and protein observed in $\mathrm{Nfic}^{-/}$pancreata extends

375 previous reports on $C D H 1$ regulation by NFIC in epithelial tissues ${ }^{19,40}$ and multiple

376 aspects of cell adhesion were revealed by GSEA in the RNA-Seq analysis. Nfic ${ }^{-/}$

377 pancreata also showed increased acinar proliferation and infiltrating leukocytes,

378 associated with an up-regulation of inflammatory transcripts. This phenotype is similar to

379 that of $\mathrm{Nr}^{2} \mathrm{a2}^{+/-23}, \mathrm{Hnf1}^{-/-36}$ and not shown), Gata6 ${ }^{-/-4}$, and Ptf1 $\mathrm{a}^{-/-25}$ pancreata. The

380 promoters of genes whose expression is up-regulated in $\mathrm{Nfic}^{-/}$pancreata were enriched

381 in motifs for NF-kB, PPARy:RXRA, REL, and NFIC itself. These findings suggest that

382 the activation of pro-inflammatory phenotypes in mice in which acinar cells fail to acquire

383 normal maturation can result from both direct (NR5A2 and GATA6) 4,7,10,12 and indirect

384 (PTF1A and NFIC) mechanisms, in agreement with the ChIP-Seq data available.

386 NFIC regulates expression of ribosomal genes and mitigates ER stress in the

387 pancreas. A hallmark of acinar cells is their prominent capacity for protein synthesis, 
388 processing, and secretion ${ }^{41}$. This is achieved through acinar-specific transcriptional 389 programs such as those driven by PTF1 ${ }^{25}$, MIST1 ${ }^{26}$, and XBP139 and - as shown here 390 - NFIC. Accordingly, a coordinated down-regulation of gene sets related to the digestive 391 process and to protein metabolism and oxidative phosphorylation occurs in adult $\mathrm{Nfic}^{-/}$ 392 pancreata. Similar changes are present in normal human pancreata displaying low NFIC 393 expression, indicating the relevance to humans. The mTOR pathway is a central actor 394 in protein biosynthesis and autophagy and, therefore, a candidate mediator of this 395 phenotype (reviewed in [33]). We found reduced levels of P-RS6K1, its substrate P-RS6, 396 and P-EIF4E, together with an up-regulation of P-ERK, in acinar cells of $\mathrm{Nfic}^{-/}$mice. 397 However, a mechanistic link between NFIC and these signaling pathways is lacking.

398 The high level of basal protein synthesis in acinar cells underlies constitutive 399 activation of the UPR to reduce ER stress ${ }^{37,42}$. We observed a down-regulation of UPR 400 gene sets and an up-regulation of classical ER stress regulators in $\mathrm{Nfic}^{-/}$pancreata. The 401 finding that NFIC - but not NR5A2 - binds the promoter of ER stress genes suggests a 402 distinct role of the former in this process. This is supported by TM-mediated ER stress 403 in 266-6 cells manipulated for NFIC gain-of-function and loss-of-function. We thus 404 conclude that NFIC mitigates ER stress in acinar cells. Previous work has shown that 405 NR5A2 is required for proper ER stress response in hepatocytes ${ }^{35}$. A re-analysis of 406 published data shows that Nfic is down-regulated in $\mathrm{Nr}^{2} \mathrm{a}^{-/}$hepatocytes in basal 407 conditions (fold change of -0.54 ) ${ }^{35}$, suggesting that the deficient response to TM in Nr5a2-

408 thepatocytes might partially occur through Nfic down-regulation. Similarly, we found that 409 NFIC is down-regulated in the pancreas upon TM-mediated ER stress (not shown), as 410 has been reported in immortalized $B$ cells ${ }^{43}$. These findings suggest that NFIC might be 411 a broad regulator of ER stress response.

412

413 NFIC is dynamically regulated during pancreatitis and cancer. A failure to achieve 414 complete acinar maturation is associated with more severe damage and delayed 415 recovery during caerulein-mediated pancreatitis, as shown upon inactivation of Gata6, 416 Mist1, and Ptf1a in the pancreas ${ }^{4,44}$. In addition, disruption of the UPR and ER stress 417 responses induces acinar damage and can lead to acute or chronic pancreatitis ${ }^{45}$. We 418 found sustained up-regulation of Ddit3/Chop and Hsp17b1 in Nfic/- pancreata upon 419 induction of a mild pancreatitis indicating enhanced ER stress in the absence of Nfic.

420 Several groups have shown that mild defects in the regulation of pancreatic 421 transcriptional programs can sensitize to pancreatitis and that TFs act as tumor 422 suppressors ${ }^{10,11,12}$. The role of NFIC in acinar cell differentiation and mitigation of ER 423 stress suggested a contribution during tumorigenesis. NFIC has been proposed to act 424 as a tumor suppressor in breast cancer, as it activates TP53, represses CCND1 and 
425 FOXF1, and is down-regulated by $c-M Y C$ and Ha-RAS oncogenes ${ }^{15-17,46,47}$. In breast 426 cancer, NFIC is down-regulated and high expression is associated with better 427 prognosis $^{16}$. Deregulation of other NFI family members has been reported in several 428 tumor types: NFIB is overexpressed in metastatic neuroendocrine lung tumors and it 429 drives metastatic progression of small cell lung cancers by increasing chromatin 430 accessibility ${ }^{48,49}$. The lack of NFIC mutations and/ or genomic alterations in human PDAC 431 (https://cancergenome.nih.gov/newsevents/newsannouncements/pancreatic_2017)

432 suggests that other mechanisms may contribute to tumor development/progression.

433 We now show that NFIC is a novel, ubiquitous, TF with tissue-specific functions 434 in the pancreas that cooperates with NR5A2 to binds target genes and controls their 435 expression in vitro and in vivo. Unlike other pancreatic TFs previously described, the role 436 of NFIC is restricted to the adult pancreas and distinctly affects RNA and protein 437 metabolism and the UPR-mediated ER stress. Mutations leading to protein misfolding, 438 the UPR, and activation of ER stress cause chronic pancreatitis and can contribute to 439 the risk of PDAC ${ }^{50}$, further supporting the role of NFIC in pancreatic homeostasis and 440 disease. 
443 Mice and experimental manipulations. The following mouse strains were used: $\mathrm{Nfic}^{-/}$

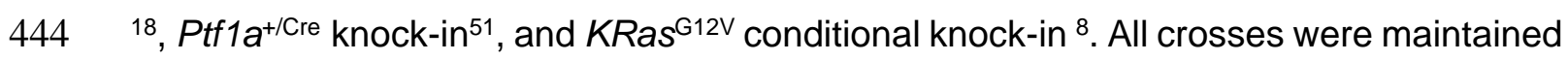
445 in a predominant C57BL/6 background. Experiments were performed using 8-12 week446 old mice of both sexes, except for glucose tolerance tests where only males were used. 447 Littermate mice were used as controls. All animal procedures were approved by local 448 and regional ethics committees (Institutional Animal Care and Use Committee and Ethics 449 Committee for Research and Animal Welfare, Instituto de Salud Carlos III) and 450 performed according to the European Union guidelines.

451 A mild acute pancreatitis was induced by 7 hourly injections of the cholecystokinin 452 analogue caerulein (Bachem) at $50 \mu \mathrm{g} / \mathrm{kg}$. In brief, animals were weighed before the 453 procedure and caerulein was administered intraperitoneally. Mice were killed by cervical 454 dislocation after $1 \& 4 \mathrm{~h}$ after the last caerulein injection or $24,48 \mathrm{~h}$ and 5 days after the 455 first caerulein injection. For the glucose tolerance test, male mice were fasted for $16 \mathrm{~h}$ 456 and basal glycaemia was measured in tail blood. Mice received a glucose solution $457(2 \mathrm{~g} / \mathrm{kg})$ administered intraperitoneally and glycaemia was measured 15, 30, 60, and 120 458 min later using an automated glucose monitor (Accu-Chek® Aviva). Fasting glucose was 459 considered as baseline (0h). The number of mice used in each experiment is shown in 460 the legend of each figure. For most experiments, $\geq 5$ mice per group were used. No specific randomization method was used.

462

463 Acinar cell isolation. Acinar cells were isolated by collagenase $P$ ( $1 \mathrm{mg} /$ pancreas) 464 digestion and maintained at $37{ }^{\circ} \mathrm{C}$ for $24 \mathrm{~h}$ in RPMI containing L-glutamine, $1 \mathrm{mM}$ of pyruvate (Sigma-Aldrich), soybean trypsin inhibitor (STI) (Gibco, 17075-029) $(0.1 \mathrm{mg} / \mathrm{ml})$ and $10 \%$ foetal bovine serum. (ref 23 )

Histology, immunofluorescence (IF) and immunohistochemical (IHC) analyses.

469 Pancreata were immediately placed in buffered formalin or $4 \%$ paraformaldehyde.

470 Histological processing was performed using standard procedures. To score damage in 471 acute pancreatitis experiments, inflammation-related histological parameters (oedema, 472 inflammatory cell infiltration, vacuolization, and acino-ductal metaplasia [ADM]) were 473 scored blindly by IC and FXR according to the grade of severity (0-3).

IF and IHC analyses were performed using $3 \mu \mathrm{m}$ sections of formalin-fixed paraffin-embedded tissues, unless otherwise indicated. After deparaffinization and 476 rehydration, antigen retrieval was performed by boiling in citrate buffer $\mathrm{pH} 6$ for $10 \mathrm{~min}$. 477 For IF, sections were incubated for $45 \mathrm{~min}$ at room temperature with $3 \% \mathrm{BSA}, 0.1 \%$ 
478 Triton X-100-PBS and then with the primary antibody overnight at $4{ }^{\circ} \mathrm{C}$. For double or

479 triple IF, the corresponding antibodies were added simultaneously and incubated

480 overnight at $4{ }^{\circ} \mathrm{C}$. Sections were then washed with $0.1 \%$ Triton-PBS, incubated with the

481 appropriate fluorochrome-conjugated secondary antibody, and nuclei were counter-

482 stained with DAPI. After washing with PBS, sections were mounted with Prolong Gold

483 Antifade Reagent (Life Technology).

484 For IHC analyses, after antigen retrieval, endogenous peroxidase was 485 inactivated with $3 \% \mathrm{H}_{2} \mathrm{O}_{2}$ in methanol for $30 \mathrm{~min}$ at room temperature. Sections were 486 incubated with $2 \%$ BSA-PBS for $1 \mathrm{~h}$ at room temperature, and then with the primary 487 antibody overnight at $4 \stackrel{\circ}{\circ}$. After washing, the Envision secondary reagent (DAKO) was 488 added for $40 \mathrm{~min}$ at room temperature and sections were washed $\times 3$ with PBS. 3,30489 Diaminobenzidine tetrahydrochloride (DAB) was used as a chromogen. Sections were 490 lightly counterstained with haematoxylin, dehydrated, and mounted. For some 491 antibodies, an automated immunostaining platform was used (Ventana Discovery XT, 492 Roche). A non-related IgG was used as a negative control. To validate the specificity of 493 anti-NFIC antibodies, $\mathrm{Nfic}^{-/}$pancreata were used as controls.

494 For CD45 quantification, whole digital slide images were acquired with an Axio 495 Scan Z1, Zeiss scanner and then captured with the Zen Software (Zeiss). Image analysis 496 and quantification were performed with the AxioVision software package (Zeiss). Briefly, 497 areas of interest (AOI) were selected for quantification and then exported as individual 498 TIFF images. CD45 staining were quantified using AxioVision 4.6 (Zeiss). Data obtained 499 were then compiled and appropriately assessed. Images containing lymph nodes, and 500 with artifactual staining or suboptimal cutting were eliminated from the analysis.

For quantification of $\mathrm{KI} 67^{+}$positive cells, at least 10 random images from each 502 pancreas were selected and only positive acinar cells were quantified. For BIP-1 503 quantification, at least 10 random images from each pancreas were taken and 504 fluorescence intensity was calculated using FIJI software (https://fiji.sc/). For semi505 quantitative analysis of KRT19 staining, intensity was scored from 0-3 by IC.

A list of antibodies used for IHC and IF is provided in Supplementary Table 3.

508 Quantitative RT-PCR (RT-qPCR). For RNA isolation, pancreata were homogenized in 509 denaturing buffer (4 M guanidine thiocyanate, $0.1 \mathrm{M}$ Trizma $\mathrm{HCl} \mathrm{pH} \mathrm{7.5,} 1 \% 2-$ 510 mercaptoethanol) and processed as described earlier ${ }^{23}$. Total RNA was treated with 511 DNase I (Ambion) for $30 \mathrm{~min}$ at $37^{\circ} \mathrm{C}$ and cDNAs were prepared according to the 512 manufacturer's specifications, using the TaqMan reverse transcription reagents (Applied 
513 Biosystems, Roche). qRT-PCR analysis was performed using the SYBR Green PCR

514 master mix and an ABIPRISM 7900HT instrument (Applied Biosystems). Expression

515 levels were normalized to endogenous $H p r t$ mRNA levels using the $\Delta \Delta C_{t}$ method. The

516 results shown are representative of at least four biological replicates. The sequence of

517 the primers used is provided in Supplementary Table 4.

518

519 Immunoprecipitation and western blotting. Pancreata were snap-frozen for protein

520 isolation. For immunoprecipitation of proteins from fresh total pancreas lysates, a piece

521 of mouse pancreas was isolated and minced in $50 \mathrm{mM}$ Tris- $\mathrm{HCl} \mathrm{pH} \mathrm{8,} 150 \mathrm{mM} \mathrm{NaCl}, 5$

$522 \mathrm{mM}$ EDTA, 0.5\% NP-40 containing $3 \times$ phosphatase inhibitor cocktail (Sigma-Aldrich)

523 and 3x EDTA-free complete protease inhibitor cocktail (Roche). Lysates were briefly

524 sonicated until the protein solution was clear, cleared for $10 \mathrm{~min}$ at $11,000 \mathrm{rpm}$ at $4{ }^{\circ} \mathrm{C}$

525 and the supernatant was recovered. Antibody-coated protein A or protein $\mathrm{G}$ dynabeads

526 (Life Technology) were used for immunoprecipitation. In brief, beads were washed three

527 times with PBS and incubated with anti-NR5A2 or normal goat IgG (Millipore) overnight

528 at $4{ }^{\circ} \mathrm{C}$. After washing three times with PBS and twice with coupling buffer $(27.3 \mathrm{mM}$

529 sodium tetraborate, $72.7 \mathrm{mM}$ boric acid), the dry beads were incubated overnight at $4{ }^{\circ} \mathrm{C}$

530 in freshly prepared $38 \mathrm{mM}$ dimethyl pimelimidate dihydrochloride in $0.1 \mathrm{M}$ sodium

531 tetraborate. Afterwards, beads were washed three times with coupling buffer and once

532 with $1 \mathrm{M}$ Tris $\mathrm{pH}$ 9. Then, $1 \mathrm{ml}$ of the Tris solution was added to the beads and incubated

533 for $10 \mathrm{~min}$ at room temperature with rotation to block amino groups and stop crosslinking.

534 Finally, beads were washed three times with storage buffer $(6.5 \mathrm{mM}$ sodium

535 tetraborate/boric acid) and stored at $4^{\circ} \mathrm{C}$ until used. Protein lysates (10-15 mg, tissues)

536 were then incubated overnight at $4{ }^{\circ} \mathrm{C}$ with antibody-coated dynabeads (Thermo Fisher

537 Scientific). Bound immune complexes were washed twice with lysis buffer containing

538 NP-40, and then eluted by boiling in $2 \times$ Laemmli buffer (10\% glycerol, $2 \%$ sodium

539 dodecyl sulphate and $0.125 \mathrm{M}$ Tris- $\mathrm{HCl} \mathrm{pH} \mathrm{6.8)} \mathrm{for} 5 \mathrm{~min}$.

$540 \quad$ For western blotting, proteins were extracted from pancreatic tissue, isolated

541 acinar cells or cultured cells using either Laemmli buffer, lysis buffer (50 mM Tris- $\mathrm{HCl} \mathrm{pH}$

$5428,150 \mathrm{mM} \mathrm{NaCl}, 5 \mathrm{mM}$ EDTA and $0.5 \% \mathrm{NP}-40$ ) or $5 \mathrm{M}$ urea, supplemented with protease

543 inhibitor and phosphatase inhibitor cocktails. Protein concentration was measured using

544 the BCA reagent (Biorad), Nanodrop or extrapolated when using Laemmli lysis buffer.

545 Proteins were resolved either by standard SDS-PAGE or 4-20\% TGX pre-cast gels

546 (Biorad) and transferred onto nitrocellulose membranes. A list of antibodies used for WB,

547 ChIP and IP is provided in Supplementary Table 3. Densitometry analysis of digitalised

548 western blotting images was performed using Fiji software (https://fiji.sc/). 
549 Chromatin immunoprecipitation (ChIP). Pancreas tissue was minced, washed with

550 cold PBS supplemented with $3 \times$ protease and phosphatase cocktail inhibitors, and then

551 fixed with $1 \%$ formaldehyde for $20 \mathrm{~min}$ at room temperature. Glycine was added to a final

552 concentration of $0.125 \mathrm{M}$ for $5 \mathrm{~min}$ at room temperature. The fixed tissue was soaked in

553 SDS buffer (50 mM Tris pH 8.1, $100 \mathrm{mM} \mathrm{NaCl}, 5 \mathrm{mM}$ EDTA and 0.5\% SDS) and

554 homogenized using a douncer. The supernatant was collected after centrifugation and

555 chromatin was sonicated with a Covaris instrument for 40 min (20\% duty cycle; $10 \%$

556 intensity; 200 cycle), yielding DNA fragments with a bulk size of 300-500 bp. Samples

557 were centrifuged to pellet cell debris. The amount of chromatin isolated was quantified

558 using Nanodrop; an aliquot of this material was used as input for final quantification.

559 Samples (0.5-1 mg of chromatin) were diluted with Triton buffer (100 mM Tris pH 8.6,

$560 \quad 0.3 \%$ SDS, $1.7 \%$ Triton X-100 and $5 \mathrm{mM}$ EDTA) to $1 \mathrm{ml}$ and pre-cleared for $2 \mathrm{~h}$ with a

561 mix of protein $A$ and $\mathrm{G}$ (previously blocked with $5 \% \mathrm{BSA}$ ) at $4{ }^{\circ} \mathrm{C}$. Antibody-coated beads

562 were added: anti-NR5A2 $(2 \mu \mathrm{g})$, anti-NFIC $(1 \mu \mathrm{g})$, and rabbit anti-PTF1A serum (1/500).

563 Non-related IgG was used as a control. After incubating for $3 \mathrm{~h}$ at $4{ }^{\circ} \mathrm{C}$ in a rotating

564 platform, beads were successively washed with $1 \mathrm{ml}$ of mixed micelle buffer $(20 \mathrm{mM}$ Tris

$565 \mathrm{pH} 8.1,150 \mathrm{mM} \mathrm{NaCl}, 5 \mathrm{mM}$ EDTA, $5 \% \mathrm{w} / \mathrm{v}$ sucrose, $1 \%$ Triton $\mathrm{X}-100$ and $0.2 \%$ SDS),

566 buffer 500 (50 mM HEPES at $\mathrm{pH}$ 7.5, 0.1\% w/v deoxycholic acid, 1\% Triton X-100, 500

$567 \mathrm{mM} \mathrm{NaCl}$ and $1 \mathrm{mM}$ EDTA), LiCl detergent wash buffer (10 mM Tris at pH 8.0, 0.5\%

568 deoxycholic acid, 0.5\% NP-40, $250 \mathrm{mM} \mathrm{LiCl}$ and $1 \mathrm{mM}$ EDTA) and TE (pH 7.5), and then

569 bound molecules were eluted by incubating overnight in elution buffer (containing $1 \%$

$570 \mathrm{SDS}$ and $100 \mathrm{mM} \mathrm{NaHCO}_{3}$ ) at $65^{\circ} \mathrm{C}$, and treated with proteinase $\mathrm{K}$ solution (10 M EDTA,

$57140 \mathrm{mM}$ Tris- $\mathrm{HCl}$ pH 6.5, $40 \mu \mathrm{g} / \mathrm{ml}$ proteinase $\mathrm{K}$ ). The eluted DNA was purified by phenol-

572 chloroform extraction. After isolation, pelleted DNA was resuspended in nuclease-free

573 water $(150 \mu \mathrm{l})$. Gene occupancy was then analysed by real-time PCR using $1 \mu \mathrm{l}$ of the

574 eluted DNA diluted in a final volume of $10 \mu$ l. The sequence of the primers used for ChIP.

575 qPCR is provided in Supplementary Table 4.

577 ChIP-Seq. ChIP sequencing libraries were prepared from purified DNA using "NEBNext 578 Ultra II DNA Library Prep Kit for Illumina" from New England BioLabs (E7645), as per 579 the manufacturers instructions. The resulting libraries were sequenced on Illumina HiSeq 580 2500, v4 Chemistry.

582 NFIC knockdown. NFIC expression was interfered in 266-6 cells using Mission shRNA 583 lentiviral constructs purchased from Sigma-Aldrich. Nfic sh1 [TRCN0000374154 584 targeting ACAGACAGCCTCCACCTACTT), Nfic sh2 (TRCN0000310992 targeting 
585 TGTGTGCAGCCGCACCATATT), and Nfic sh3 (TRCN0000301779, targeting 586 GATGGACAAATCTCCATTCAA)]. Control cells were transformed using lentiviral 587 particles transducing the scrambled vector CCGGCAACAAGATGA 588 AGAGCACCAACTCGAGTTGGTGCTCTTCATCTTGTTGTTTTT (shNT).

589 To produce lentiviral particles, HEK293-FT cells (ATCC) were allowed to reach $59050 \%$ of confluence and transfected with $15 \mu \mathrm{g}$ of shNT, Nfic sh1, Nfic sh2 or Nfic sh3 591 plasmids together with $8 \mu \mathrm{g}$ of psPAX and $2 \mu \mathrm{g}$ of PCMV-VSVG helper plasmids using $592 \mathrm{CaCl}_{2} 2 \mathrm{M}$ HBSS. After $12 \mathrm{~h}$, the supernatant was collected and replaced with $5 \mathrm{ml}$ of 593 fresh medium. The supernatant was collected $24 \mathrm{~h}, 48 \mathrm{~h}$ and $72 \mathrm{~h}$ after transfection. The 594 medium was filtered ( $0.45 \mu \mathrm{m}$ pore) and added to $266-6$ cells (at $50-60 \%$ of confluence); $5951 \mu \mathrm{g} / \mathrm{mL}$ of Polybrene (hexadimethrine bromide, Sigma-Aldrich 107689) was added to 596 increase infection efficiency. After 2-3 rounds of infection, the supernatant was removed 597 and replaced with fresh medium. One day later, puromycin (1-2 $\mu \mathrm{g} / \mathrm{ml})$ (Sigma-Aldrich) 598 was added and two days later, the medium was replaced.

600 NFIC lentiviral overexpression. Nfic-HA tagged cDNA was purchased from Addgene 601 (https://www.addgene.org/31403/) and subcloned into the lentiviral vector pLVX-puro 602 using $\mathrm{Xhol}$ and $\mathrm{Xbal}$. Insert sequence was checked using enzymatic digestion and 603 Sanger sequencing. The production of lentiviral particles and cellular infection were 604 performed as described earlier. The medium from the transfectants was collected $24 \mathrm{~h}$, $60548 \mathrm{~h}$ and $72 \mathrm{~h}$ after transfection. Subsequently, 266-6 cells were infected using Polybrene 606 as described earlier. After selection with puromycin for 24-48h, resistant 266-6 cells were 607 collected for RNA and protein analysis.

608

609 Tunicamycin (TM) treatment. 266-6 cells were seeded until they reached $70 \%$ 610 confluence. After pilot dose-response experiments, a concentration of $10 \mathrm{nM}$ was 611 chosen; cells treated with TM or vehicle were collected at various time-points for RNA 612 and protein analysis.

613

614 RNA-Seq libraries preparation and analysis. RNA from wild type and Nfic-/- pancreata 615 was isolated as described above and sequenced on Illumina platform. RNA-seq data for 616 Nr5a2 (GSE34030), Mist1 (GSE86288) were downloaded from SRA. Data were 617 analysed using the nextpresso pipeline http://bioinfo.cnio.es/nextpresso/). 
619 Comparison of gene expression in normal human pancreata according to NFIC 620 transcript levels was performed using RNA-Seq data from GTEX website

621 (https://gtexportal.org/home/datasets, version 6) ( $n=171)$, as described21. The

622 expression data matrix was sorted by NFIC expression levels taking the 10 individuals 623 scoring highest and lowest NFIC expression levels (NFIChigh, NFIClow). Differential 624 expression analysis using the DEGseq package of $R$ 625 (https://bioconductor.org/packages/release/bioc/html/DEGseq.html). MA-plot-based 626 method with Random Sampling model -MARS- (Wang et al. 2009) was applied and only 627 genes with significance $\mathrm{P}<0.001$ were used in the analysis. All data have been deposited 628 in GEO with accession number GSE126907.

630 RNA-seq and data processing. RNA-Seq of pancreata from wild type mice during 631 pancreatitis was analysed as previously described in [23] and is available under 632 GSE84659 (https://www.ncbi.nlm.nih.gov/geo/query/acc.cgi?acc=GSE84659).

633 Briefly, RNA from wild type and Nfic-/- pancreata was isolated as described above. Data 634 were analysed using the nextpresso pipeline http://bioinfo.cnio.es/nextpresso/). Tophat 635 was used for alignment (tophat-2.0.10.Linux_x86_64) using the following parameters: 636 useGTF="true" nTophatThreads="1" maxMultihits="5" readMismatches="1", 637 segmentLength="19", $\quad$ segmentMismatches="1", $\quad$ spliceMismatches="0", 638 reportSecondaryAlignments="false" bowtie="1", readEditDist="2" readGapLength="2" 639 referencelndexing="false", --no-coverage-search. Gene expression was quantified using 640 cufflinks (version 2.2.1) using the following parameters: useGTF="true" nThreads="1" 641 fragBiasCorrect= "true", multiReadCorrect="false" library Normalization Method= 642 "classic-fpkm" max Bundle Frags="5000000000", normalization= "compatibleHits", no 643 Effective Length Correction="true" no Length Correction="false". Differential expression 644 analysis was done using cuffdiff (version 2.2.1) using the following parameters: 645 useCuffmergeAssembly="false", $\quad$ nThreads="1", $\quad$ fragBiasCorrect="true", 646 multiReadCorrect="false", libraryNormalization Method="geometric" FDR="0.05" 647 minAlignmentCount="10", seed="123L" FPKMthreshold="2" , maxBundleFrags= 648 "5000000000", noEffectiveLengthCorrection="true", noLengthCorrection="false" 649 dispersion Method= "pooled". Normalised expression across all samples was calculated 650 using cuffnorm (vs 2.2.1) using the following parameters: 651 useCuffmergeAssembly="false", nThreads="1", output Format="simple-table" 652 libraryNormalizationMethod="geometric", seed="123L" normalization= "compatibleHits". 653 BEDTools-Version-2.16.2 and samtools-0.1.19; bowtie-1.0.0 were also used to execute 654 the software shown above. 
To analyze gene expression in normal human pancreas, RNA-Seq data was downloaded from GTEX website (https://gtexportal.org/home/datasets, version 6); 171 samples were used. The expression data matrix was sorted by NFIC expression levels to then take the 10 top and bottom individuals of expression (NFIChigh, NFIClow) top 660 vs. bottom 10 individuals. Differential expression analysis using the DEGseq package of 661 R (https://bioconductor.org/packages/release/bioc/html/DEGseq.html). MA-plot-based 662 method with Random Sampling model -MARS- (Wang et al. 2009) was applied and only genes with significance $\mathrm{P}<0.001$ were used in the analysis.

664

665 Principal component analysis (PCA). The Pearson correlation was calculated from the expression value (expressed as fragments per kilobase of transcript per million mapped reads) of each gene for each sample by using the 'cor' command in R (https://www.rproject.org/). Principal component analysis was performed using the 'prcomp' command in $\mathrm{R}$, from the correlation value of each sample.

Gene Set Enrichment Analysis (GSEA). A ranking metric $[-\log 10(p$ value)/sign(log2FoldChange)] was used to generate a ranked gene list from the DEseq output. The list of pre-ranked genes was then analysed with using the molecular signature dataset of GSEA for Gene Ontology (GO), KEGG, REACTOME, HALLMARKS or CANONICAL PATHWAYS databases as described in the Figure legends and the text.

676 Significantly enriched terms were identified using a false discovery rate (FDR) $q$ value of $<0.25$.

NFIC ChIP-Seq. Chromatin from mouse pancreas tissue was extracted and processed as described above. For ChIP sequencing, libraries were prepared from purified DNA using "NEBNext Ultra II DNA Library Prep Kit for Illumina" from New England BioLabs (NEB, \#E7645), as per the manufacturers' instructions. The resulting libraries were sequenced on Illumina HiSeq 2500, v4 Chemistry.

685 ChIP-seq data processing. Data from NR5A2 ChIP-Seq in adult pancreata 686 (SRR389293, SRR389294), NR5A2 ChIP-Seq in ES cells (GSM470523, GSM470524), 687 PTF1A ChIP-Seq in adult pancreata (GSM2051452, GSM2051453), and MIST1 ChIP688 Seq in adult pancreata (GSM2299654,GSM2299654, GSM2299655) were downloaded 689 from the Gene Expression Omnibus website (https:/www.ncbi.nlm.nih.gov/geo/) and 690 analysis was performed as described ${ }^{23}$. Briefly, after the quality check by fastqc (v.0.9.4, 
691 Babraham Bioinformatics), the alignment and peak calling for the ChIP-seq data was

692 performed using RUbioSeq + pipeline ${ }^{52}$. Merging of replicate peaks and peak annotation

693 was done using HOMER. Peak calling, annotation and motif enrichment was identified

694 using HOMER (Heinz et al., 2010; http://homer.ucsd.edu/homer/). Reads were

695 directionally extended to $300 \mathrm{bp}$ and, for each base pair in the genome, the number of

696 overlapping sequence reads was determined and averaged over a 10-bp window to

697 create a wig file to visualize the data in the University of California Santa Cruz (UCSC)

698 genome browser.

699 NFIC ChIP-Seq data using GM12878, ECC1, HepG2, SK-N-SH and K562 cells

700 were downloaded from (https://www.encodeproject.org/targets/NFIC-human/). ChIP-

701 Seq peaks were analysed using Peak Analyser_1.4, using and Nearest Transcription

702 Start Site parameter was used to annotate the genomic location of peaks. More than

$70395 \%$ of the target genes identified in the replicate with lowest number of target genes

704 were included in the replicate with highest number. Among the two replicates, the one

705 with highest number of identified target genes was taken: replicate 1 of NFIC ChIP-Seq

706 in GM12878, NFIC ChIP-Seq in HepG2 and NFIC ChIP-Seq in SK-N-SH and replicate 2

707 of NFIC ChIP-Seq in ECC1 cell line.

709 Other statistical analyses. Comparisons of quantitative data between groups were was

710 performed using one-sided Mann-Whitney $U$ test in all cases for which there was a prior

711 hypothesis, except for the data shown in Figure $6 \mathrm{D}$, E where a prior existed. Box plots

712 represent the median and second and third quartiles (interquartile range, IQR) of the

713 data. Error bars are generated by $R$ software and represent the highest and lowest data

714 within 1.5× IQR range. All statistical analyses were performed with Excel, $\mathrm{R}$ software,

715 https://ccb-compute2.cs.uni-saarland.de/wtest/ $0 r$

716 https://www.medcalc.org/calc/comparison of proportions.php. The random list of

717 genes were generated using https://www.dcode.fr/random-selection and

718 http://www.molbiotools.com/randomgenesetgenerator.html websites. Duplicated

719 transcripts in RNA-Seq data were deleted for analysis. Dotted line refer to threshold for

720 statistical significance $(-\log 10[0.25]=0.60)$ or $(-\log 10[0.05]=1.30$. Two-sided Mann-

721 Whitney $\mathrm{U}$ test was used unless otherwise indicated; $\mathrm{P}<0.05\left({ }^{*}\right) ; \mathrm{P}<0.01\left(^{* *}\right)$ 
We thank A. Efeyan, N. Djouder, and P. Martinelli for valuable discussions; M.

728 Soengas and B. Bréant for providing antibodies; J. Perales and F. Al-Shahrour for help 729 handling the GTEX dataset; R.M. Gronostajski, M. Barbacid, and C.W. Wright for 730 providing mice; M. Barba, C. Yolanda and T. Lobato for technical assistance; and the 731 Core Facilities and Bioinformatics Unit of Spanish National Cancer Research Center 732 (CNIO) for support. 


\section{FIGURE LEGENDS}

734 Figure 1. The NR5A2 is a novel acinar regulator. (A) HOMER de novo motif analysis 735 for NR5A2, PTF1A and GATA6 ChIP-Seq in mouse pancreata showing enrichment in $736 \mathrm{NF1/NFI} \mathrm{motifs.} \mathrm{(B)} \mathrm{Expression} \mathrm{of} \mathrm{NFI} \mathrm{transcripts} \mathrm{in} \mathrm{mouse} \mathrm{(upper} \mathrm{panel)} \mathrm{or} \mathrm{human}$ 737 (lower panel) pancreata assessed by RNA-Seq showing that NFIC is the family member 738 expressed at highest levels. (C) Immunoprecipitation-western blotting analysis showing 739 that NFIC and NR5A2 are part of the same complex in adult, but not in embryonic, 740 pancreas. (D) Spamo analysis showing distance conservation of the NR5A2 and NFIC 741 motifs in the regions bound by NR5A2. (E) ChIP-qPCR of NR5A2 and PTF1A binding at 742 the Nfic promoter (one region in NR5A2 peak1 and two regions in NR5A2 peak3); 743 controls as in panel $F$ ( $n=4 /$ group). ( $F$ ) ChIP-qPCR of NR5A2 and NFIC binding to the 744 promoter of digestive enzyme genes and NrOb2, compared to a control (Neg) region 745 (normalized to unrelated IgG) ( $\mathrm{n}=6$ /group). (G) IHC analysis of NFIC in normal adult 746 mouse pancreas showing higher expression in acinar cells and lower expression in 747 endocrine and ductal cells (insets). (H) Lentiviral Nfic knockdown in 266-6 cells showing 748 reduced expression of transcripts coding for digestive enzyme transcripts and pancreatic 749 TFs (RT-qPCR) (left panel); western blotting analysis of the corresponding samples 750 interfered with non-targeting (NT) or Nfic-targeting shRNAs $(n=3)$.

752 Figure 2. NFIC is required for normal acinar cell differentiation. (A) RT-qPCR 753 showing reduced expression of transcripts coding for digestive enzymes and pancreatic 754 TF in Nfic-/- pancreata. (B) Western blotting showing reduced expression of digestive 755 enzymes in $\mathrm{Nfic}^{-/}$pancreata ( $\mathrm{n}=7$ /group). (C) Densitometric quantification of panel 3B: 756 band intensity normalised to loading control, relative to wild-type pancreata. (D) 757 Comparison of the overlap of DEG in the pancreas of $\mathrm{Nfic}^{-/-}$vs. that of mice lacking 758 NR5A2, PTF1A, and MIST1 (details in text). Statistics: two-tailed Student-T test. 759 Significant overlap is shown for down-regulated genes compared to a random list of 760 genes. "N-1" chi-squared test was used to calculate statistical significance.

Figure 3. NFIC binds to genomic regions associated to genes involved in acinar differentiation, ER stress, UPR, and inflammation. (A) De novo motif analysis of NFIC ChIP-Seq showing NFI as the top-motif. (B) Distribution of NFIC ChIP-Seq peaks showing enrichment in regions close to the TSS (left) and the corresponding enrichment of the NFI, ELK and CTCF motifs (right). (C) Venn diagram showing the overlap between genes with an NFIC peak and those de-regulated in the Nfic-/- pancreas showing a 
769 ChIP-Seq peaks based on score intensity and the overlap with genes de-regulated in

770 Nfic-/- pancreata showing slight greater overlap in Q1, Q2 for the down-regulated genes.

771 (E,F) Motif analysis of genes with an NFIC peak that are down-regulated (E) or up772 regulated $(\mathrm{F})$ in Nfic-/- pancreata; NFI is the top motif in both groups. $(\mathrm{G}, \mathrm{H})$ Gene set 773 enrichment analysis of genes bound by NFIC and down-regulated (G) or up-regulated $774(\mathrm{H})$ in Nfic-/- pancreata showing down-regulation of bona fide acinar, ribosomal, and 775 metabolic genes; and up-regulation of inflammatory, UPR, and ER stress genes. Boxes 776 show representative examples of genes included in each analysis. (I) UCSC browser 777 shots of NFIC ChIP-Seq showing enrichment for Cela2a, NrOb2, Bhlha15, Pparg, Cfi and 778 Rara.

780 Figure 4. NFIC regulates protein biosynthesis in the pancreas. (A) IF analysis of reactivity with an antibody recognizing $5.8 \mathrm{~S}$ rRNA and $\mathrm{CDH} 1$ shows decreased expression of both in $\mathrm{Nfic}^{--}$acinar cells. (B) Quantification of panel 4A. (C) Western blotting showing mTOR and ERK pathway signaling changes in $\mathrm{Nfic}^{-/}$pancreata. (D) IF displaying down-regulation of CPA and P-RS6 in Nfic ${ }^{--}$pancreata $(n=2)$. (E,F) IHC analysis $(E)$ of $P$-ERK and quantification $(F)$ showing increased number of positive acinar cells in $\mathrm{Nfic}^{-/}$pancreata $(\mathrm{n}=2)$. (G) Boxplot plot showing the relationship between the expression of up-regulated, down-regulated, or a random set of genes, in control $\mathrm{Nfic}^{+/+}$ vs. $\mathrm{Nfic}^{-/}$mice and in histologically normal human pancreatic tissues samples (top 10 low- vs. top 10 high-expressing NFIC mRNA levels, as determined by RNA-Seq analysis $\left[N F I C^{\text {low }}\right.$ vs. $\left.\left.N F I C^{\text {high }}\right]\right)$. Data shows the concordant pattern between down-regulated genes in $\mathrm{Nfic}^{-/-}$mice and NFIClow human pancreata. "N-1" chi-squared test was used to calculate statistical significance. P-value was calculated comparing to a random gene list. (H) GSEA for genes that are concurrently down-regulated in $\mathrm{Nfic}^{-/}$vs. wild type pancreata and in NFIClow vs. NFIC high human pancreata. Genes were computed with KEGG data sets showing the similarities with those gene sets under-represented in NficI- mice (G). (I) Bar plot displaying the down-regulation of ribosomal genes in NFIClow vs. $N F I C^{\text {high }}$ human pancreata $(\mathrm{P}<0.001)$.

Figure 5. NFIC regulates aspects of UPR and ER stress resolution. (A) GSEA

800 analysis of the UPR and ER stress gene sets ${ }^{16}$ down-regulated in $\mathrm{Nfic}^{-/}$pancreata and up-regulation of UPR. (B) RT-qPCR showing expression of spliced Xbp1, Chop (Ddit3),

802 Bip-1(Hspa5), and Hsp90b1 in wild-type and Nfic/- pancreata (n>4/group). (C) Western 803 blotting showing up-regulation of BIP-1 and CHOP in $\mathrm{Nfic}^{-/}$pancreata ( $\mathrm{n}=7 /$ group). (D) 804 Densitometric quantification of data in panel 5C. (E) ChIP-qPCR showing binding of 805 NFIC, but not NR5A2, to the promoters of Hspa5/Bip-1, Ddit3 and Hsp90aa1. (F) Up- 
806 regulation of BIP-1 and CHOP in 266-6 cells treated with TM upon Nfic knock-down. (G)

807 Quantification of data shown in 5F. (H) Reduced BIP-1 and CHOP expression in control

808 and NFIC-overexpressing 266-6 cells treated with TM. (I) Quantification of data shown 809 in $5 \mathrm{H}$.

810

811 Figure 6. NFIC is dynamically regulated and required for a homeostatic response

812 during caerulein pancreatitis. (A) RNA-Seq analysis of Nfic, Nr5a2, and Ptf1a

813 expression in wild-type mice upon induction of a mild acute pancreatitis ( $n=3$ /group).

814 Significance was calculated compared to expression at Oh. (B) IF analysis of NFIC and

815 PTF1A upon pancreatitis induction showing NFIC down-regulation ( $n=4 /$ group). (C)

816 Quantification of $\mathrm{PTF} \mathrm{A}^{+}$and $\mathrm{NFIC}^{+}$cells in wild-type mice during pancreatitis. (D)

817 Histological analysis of wild-type and $\mathrm{Nfic}^{-/}$pancreata $24 \mathrm{~h}, 48 \mathrm{~h}$, and 5 days after the

818 induction of pancreatitis showing increased damage in mutant mice. (E) Pancreatitis

819 scoring indicates more severe damage in $\mathrm{Nfic}^{-/}$pancreata at $48 \mathrm{~h}$ and day 5

820 ( $\mathrm{n}>4$ /condition). ( $\mathrm{F}-\mathrm{H}) \mathrm{IHC}$ reveals increased expression of KRT19, a higher number of

$821 \mathrm{KI}^{+} 7^{+}$acinar cells, and increased infiltration by $\mathrm{CD}^{+} 5^{+}$cells in $\mathrm{Nfic}^{-/}$pancreata.

822 Representative images (F). Quantification of CD45 (G), KRT19 and Ki67 expression (H).

823 was subjectively scored (0-3) in Nfic-/- pancreata ( $n \equiv 4 /$ group).

826 Figure 7. NFIC restrains formation of preneoplastic lesions in the pancreas. (A)

827 IHC analysis of NFIC expression shows down-regulation in PanINs (right) and tumor

828 cells compared to adjacent normal acinar cells (left). (B) NFIC mRNA analysis of tumor 829 samples and normal adjacent tissue assessed by microarrays (Janky et al., 2016) 830 showing reduced expression in tumor samples. (C,D) IHC analysis of NFIC expression 831 in human PDAC specimens showing reduced expression in tumoral cells (arrow) 832 compared to normal adjacent tissue or stromal cells (arrowheads). (E,F) Histological 833 analysis of the pancreas of KRas ${ }^{G 12 V}$ or KRas ${ }^{G 12 V} ; N^{\prime} i^{-1} 14-20$ week old mice showing 834 increased number of PanINs and area occupied by pre-neoplastic lesions 835 ( $\mathrm{n}>5$ /genotype).

836

837

838 


\section{REFERENCES}

1. Pandol SJ. The exocrine pancreas. San Rafael (CA), Morgan \& Claypool Life Sciences 2010.

2. Rose SD, Swift GH, et al. The role of PTF1-P48 in pancreatic acinar gene expression. J Biol Chem 2001; 276:44018-44026.

3. Beres TM, Masui T, et al. PTF1 is an organ-specific and Notch-independent basic helix-loop-helix complex containing the mammalian Suppressor of Hairless (RBP-J) or its paralogue, RBP-L. Mol Cell Biol 2006; 26:117-130.

4. Martinelli $P$, Cañamero $M$, et al. Gata6 is required for complete acinar differentiation and maintenance of the exocrine pancreas in adult mice. Gut 2013; 62:1481-1488.

5. Pin CL, Rukstalis JM, et al. The bHLH transcription factor Mist1 is required to maintain exocrine pancreas cell organization and acinar cell identity. J Cell Biol 2001; 155:519-530.

6. Holmstron SR, Deering T, et al. LRH-1 and PTF1-L coregulate an exocrine pancreas-specific transcriptional network for digestive function. Genes \& Dev $2011 ; 25: 1674-1679$.

7. Hale MA, Swift GH, et al. Development. The nuclear hormone receptor family member NR5A2 controls aspects of multipotent progenitor cell formation and acinar differentiation during pancreatic organogenesis. Development 2014; 141:3123-3133.

8. Guerra C, Schuhmacher AJ, et al. Chronic pancreatitis is essential for induction of pancreatic ductal adenocarcinoma by K-Ras oncogenes in adult mice. Cancer Cell 2007; 11:291-302.

9. Kopp JL, von Figura G, et al. Identification of SOX9-dependent acinar-to-ductal reprogramming as the principal mechanism for initiation of pancreatic ductal adenocarcinoma. Cancer Cell 2012; 22:737-750.

10. Flandez M, Cendrowski J, et al. Nr5a2 heterozygosity sensitises to, and cooperates with, inflammation in KRas(G12V)-driven pancreatic tumourigenesis. Gut 2014; 63:647-655.

11. Martinelli $P$, Madriles $F$, et al. The acinar regulator Gata6 suppresses KrasG12V-driven pancreatic tumorigenesis in mice. Gut 2016; 65:476-486. constrains oncogenic Kras-mediated pancreatic neoplastic initiation. Gut 2014; 63:656-664. development. Gene 2000; 249:31-45. 
14. Kannius-Janson M, Johansson EM, et al. Nuclear factor 1-C2 contributes to the tissue-specific activation of a milk protein gene in the differentiating mammary gland. J Biol Chem 2002; 277: 17589-17596.

15. Eeckhoute J, Carroll JS, et al. Cell-type-specific transcriptional network required for estrogen regulation of cyclin D1 and cell cycle progression in breast cancer. Genes Dev 2006; 20: 2513-2526.

16. Nilsson J, Helou K, et al. Nuclear Janus-activated kinase 2/nuclear factor 1-C2 suppresses tumorigenesis and epithelial-to-mesenchimal transition by repressing Forkhead box F1. Cancer Res 2010; 70:2020-2029.

17. Johansson EM, Kannius-Janson M, et al. The p53 tumor suppressor gene is regulated in vivo by nuclear factor 1-C2 in the mouse mammary gland during pregnancy. Oncogene 2003; 22:6061-6070.

18. Steele-Perkins G, Butz KG, et al. Essential role for NFI-C/CTF transcriptionreplication factor in tooth root development. Mol Cell Biol 2003; 23:1075-84.

19. Lee HK, Lee DS. Nuclear Factor I-C (NFIC) regulates Dentin Sialophosphoprotein (DSPP) and E-cadherin via control of Krüppel-like Factor 4 (KLF4) during dentinogenesis. J Biol Chem 2014; 289:28225-28236.

20. Zhou J, Wang S, et al. Nuclear factor I-C reciprocally regulates adipocyte and osteoblast differentiation via control of canonical Wnt signaling. FASEB J 2017; 31:1939-1952.

21. Liu Y, Feng J, et al. An Nfic-hedgehog signaling cascade regulates tooth root development. Development 2015; 142:3374-3382.

22. Heng JC, Feng B, et al. The nuclear receptor Nr5a2 can replace Oct4 in the reprogramming of murine somatic cells to pluripotent cells. Cell Stem Cell 2010; 6:167-174.

23. Cobo I, Martinelli $P$, et al. Transcriptional regulation by NR5A2 links differentiation and inflammation in the pancreas. Nature 2018; 554:533-537.

24. Cendrowski J, Lobo VJ, et al. Mnk1 is a novel acinar cell-specific kinase required for exocrine pancreatic secretion and response to pancreatitis in mice. Gut 2015: 64;937-947.

25. Hoang CQ, Hale MA, et al. Transcriptional Maintenance of Pancreatic Acinar Identity, Differentiation, and Homeostasis by PTF1A. Mol Cell Biol 2016; 36:3033-3047.

26. Jiang M, Azevedo-Pouly AC, et al. MIST1 and PTF1 Collaborate in FeedForward Regulatory Loops That Maintain the Pancreatic Acinar Phenotype in Adult Mice. Mol Cell Biol 2016. 36:2945-2955. 
912

913

914

915

916

917

918

919

920

921

922

923

924

925

926

927

928

929

930

931

932

933

934

935

936

937

938

939

940

941

942

943

944

945

946

947

948

27. Biasci D, Smoragiewicz M, et al. CXCR4 inhibition in human pancreatic and colorectal cancers induces an integrated immune response. Proc Natl Acad Sci U S A. 2020 17;117:28960-28970.

28. Heinrich EL, Lee W, et al. Chemokine CXCL12 activates dual CXCR4 and CXCR7-mediated signaling pathways in pancreatic cancer cells. J Transl Med 2012;10: 68.

29. Perera RM, Bardeesy N. Pancreatic Cancer Metabolism: Breaking It Down to Build It Back Up. Cancer Discov. 2015;5:1247-1261.

30. Qin C, Yang G, et al. Metabolism of pancreatic cancer: paving the way to better anticancer strategies. Mol Cancer. 2020;19:50.

31. García-Costela M, Escudero-Feliú J, et al. Circadian Genes as Therapeutic Targets in Pancreatic Cancer. Front Endocrinol (Lausanne). 2020;11:638.

32. Kobayashi $\mathrm{H}$, Spilde TL, et al. Retinoid signaling controls mouse pancreatic exocrine lineage selection through epithelial-mesenchymal interactions. Gastroenterology. 2002;123:1331-1340.

33. Laplante M and Sabatini DM. mTOR signaling in growth control and disease. Cell 2012; 149:274-293.

34. Senft D and Ronai ZA. UPR, autophagy, and mitochondria crosstalk underlies the ER stress response. Trends Biochem Sci 2015; 40:141-148.

35. Mamrosh JL, Lee JM, et al. Nuclear receptor LRH-1/NR5A2 is required and targetable for liver endoplasmic reticulum stress resolution. Elife 2014; 3:e01694.

36. Molero X, Vaquero EC, et al. Gene expression dynamics after murine pancreatitis unveils novel roles for Hnf1alpha in acinar cell homeostasis. Gut 2012; 61:1187-1196.

37. Kubisch $\mathrm{CH}$, Logsdon CD. Secretagogues differentially activate endoplasmic reticulum stress responses in pancreatic acinar cells. Am J Physiol Gastrointest Liver Physiol 2007; 292:G1804-G1812.

38. Janky R, Binda MM, et al. Prognostic relevance of molecular subtypes and master regulators in pancreatic ductal adenocarcinoma. BMC Cancer 2016; 16:632.

39. Lee $\mathrm{AH}, \mathrm{Chu} \mathrm{GC}$, et al. XBP-1 is required for biogenesis of cellular secretory machinery of exocrine glands. EMBO J 2005; 24:4368-4380.

40. Lee HK, Lee DS, et al. Nuclear factor I-C regulates E-cadherin via control of Klf4 in breast cancer. BMC Cancer 2015; 15:113.

41. Case RM. Synthesis, intracellular transport and discharge of exportable proteins in the pancreatic acinar cell and other cells. Biol Rev Camb Philos Soc 1978; 53:211-354. 
42. Drummond A and Wilke CO. The evolutionary consequences of erroneous protein synthesis. Nat Rev Genet 2009; 10:715-724.

43. Dombroski BA, Nayak RR, et al. Gene expression and genetic variation in response to endoplasmic reticulum stress in human cells. Am J Hum Genet 2010; 86:719-729.

44. Kowalik AS, Johnson CL, et al. Mice lacking the transcription factor Mist1 exhibit an altered stress response and increased sensitivity to caeruleininduced pancreatitis. Am J Physiol Gastrointest Liver Physiol. 2007 ;292:G1123-1132.

45. Sah RP, Garg SK, et al. Endoplasmic reticulum stress is chronically activated in chronic pancreatitis. J Biol Chem 2014; 289:27551-27561.

46. Yang BS, Gilbert JD, et al. Overexpression of Myc suppresses CCAAT transcription factor/nuclear factor 1-dependent promoters in vivo. Mol Cell Biol 1993; 13:3093-3102.

47. Nebl G, Mermod N, et al. Post-transcriptional down-regulation of expression of transcription factor NF1 by Ha-ras oncogene. J. Biol Chem 1994; 269:73717378.

48. Denny SK, Yang D, et al. Nfib Promotes Metastasis through a Widespread Increase in Chromatin Accessibility. Cell 2016; 166:328-342.

49. Semenova EA, Kwon MC, et al. Transcription Factor NFIB Is a Driver of Small Cell Lung Cancer Progression in Mice and Marks Metastatic Disease in Patients. Cell Rep 2016; 16:631-643.

50. Lukas J, Pospech J. et al. Role of endoplasmic reticulum stress and protein misfolding in disorders of the liver and pancreas. Adv Med Sci 2019; 64:315323.

51. Kawaguchi $Y$, Cooper B, et al. The role of the transcriptional regulator Ptf1a in converting intestinal to pancreatic progenitors. Nat Genet 2002; 32:128-134.

52. Rubio-Camarillo M, López-Fernández H, et al. RUbioSeq+: A multiplatform application that executes parallelized pipelines to analyse next-generation sequencing data. Comput Methods Programs Biomed. 2017; 138:73-81. 
A

\begin{tabular}{|c|c|c|c|c|}
\hline de novo motifs & $\begin{array}{l}\text { best } \\
\text { match }\end{array}$ & $\begin{array}{c}\% \\
\text { target }\end{array}$ & $\begin{array}{c}\% \\
\text { backg. }\end{array}$ & $\begin{array}{l}-\log _{10} \\
\text { (FDR) }\end{array}$ \\
\hline GACCITGACE & NR5A2 & 38.85 & 9.39 & 2926 \\
\hline 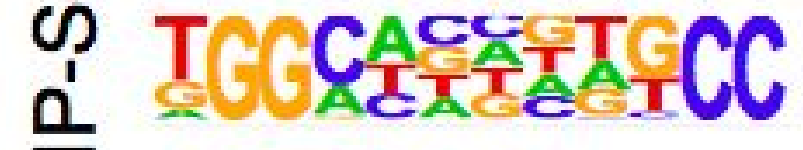 & NF1 & 21.95 & 6.39 & 1212 \\
\hline ACTTGCCA & NFIC & 36.65 & 19.77 & 720 \\
\hline 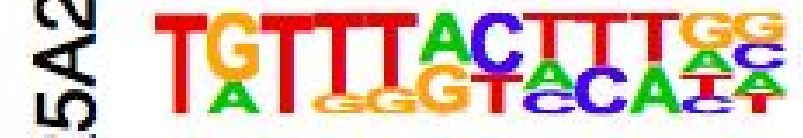 & FOXM1 & 23.27 & 11.32 & 534 \\
\hline 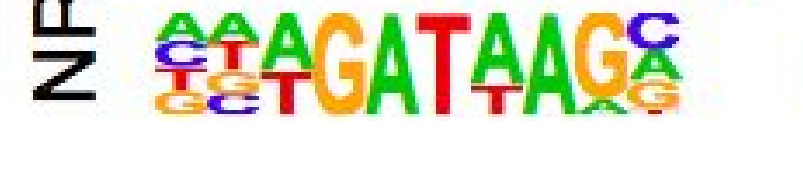 & GATA6 & 13.06 & 5.03 & 451 \\
\hline ఫ ACACCTCTTC & PTF1A & 63.54 & 23.07 & 1182 \\
\hline ITTCCCACGE & RBPJL & 46.41 & 19.89 & 566 \\
\hline 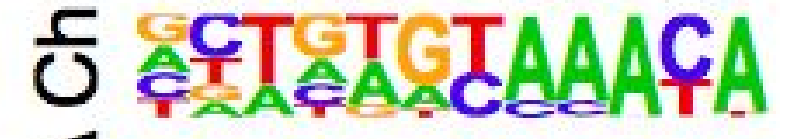 & bHLH & 16.62 & 4.69 & 315 \\
\hline 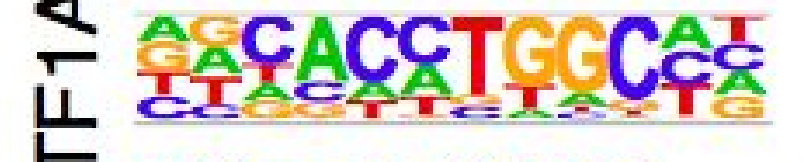 & NFIX & 14.55 & 7.00 & 109 \\
\hline 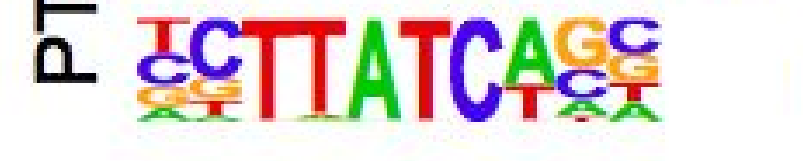 & GATA4 & 19.85 & 12.18 & 76 \\
\hline 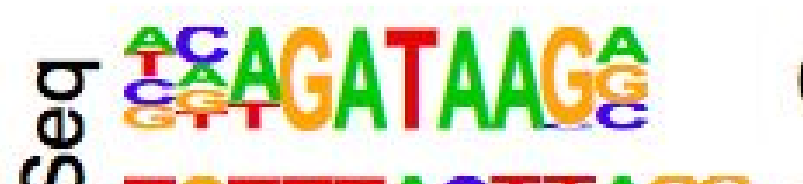 & GATA6 & 53.13 & 11.30 & 3104 \\
\hline 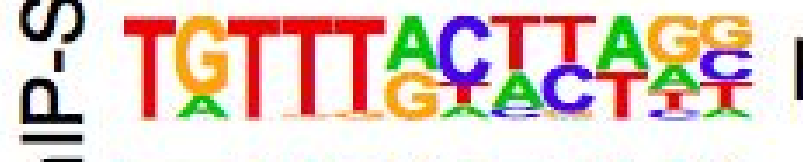 & FOXA2 & 32.01 & 15.71 & 486 \\
\hline IIAATC ATTAAC । & HNF1B & 7.12 & 2.00 & 241 \\
\hline [CCCAEGT & NF1 & 47.66 & 33.98 & 236 \\
\hline AACAECTG & PTF1A & 32.48 & 21.33 & 199 \\
\hline
\end{tabular}

E

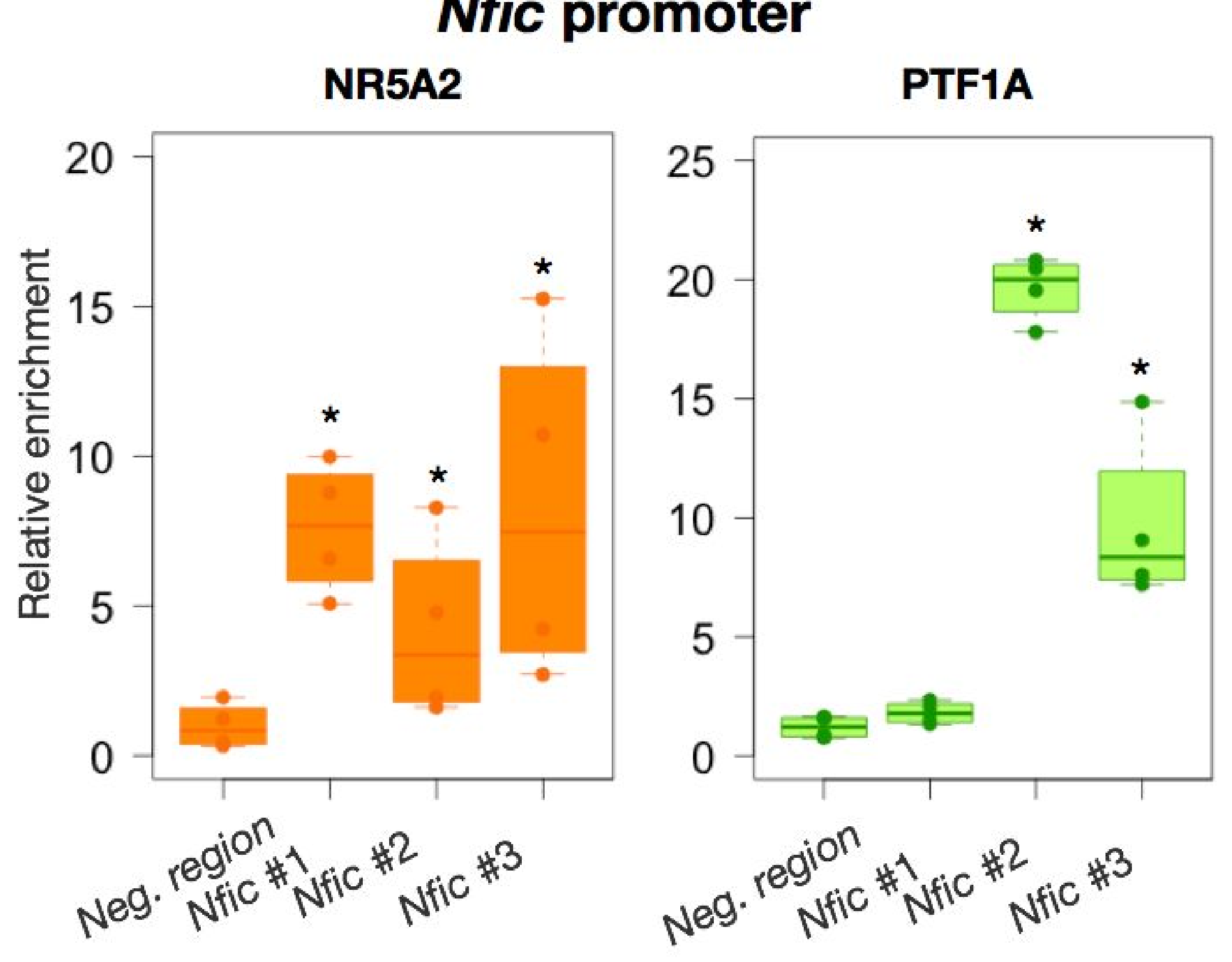

\section{Bulk RNA-Seq (Mouse)}

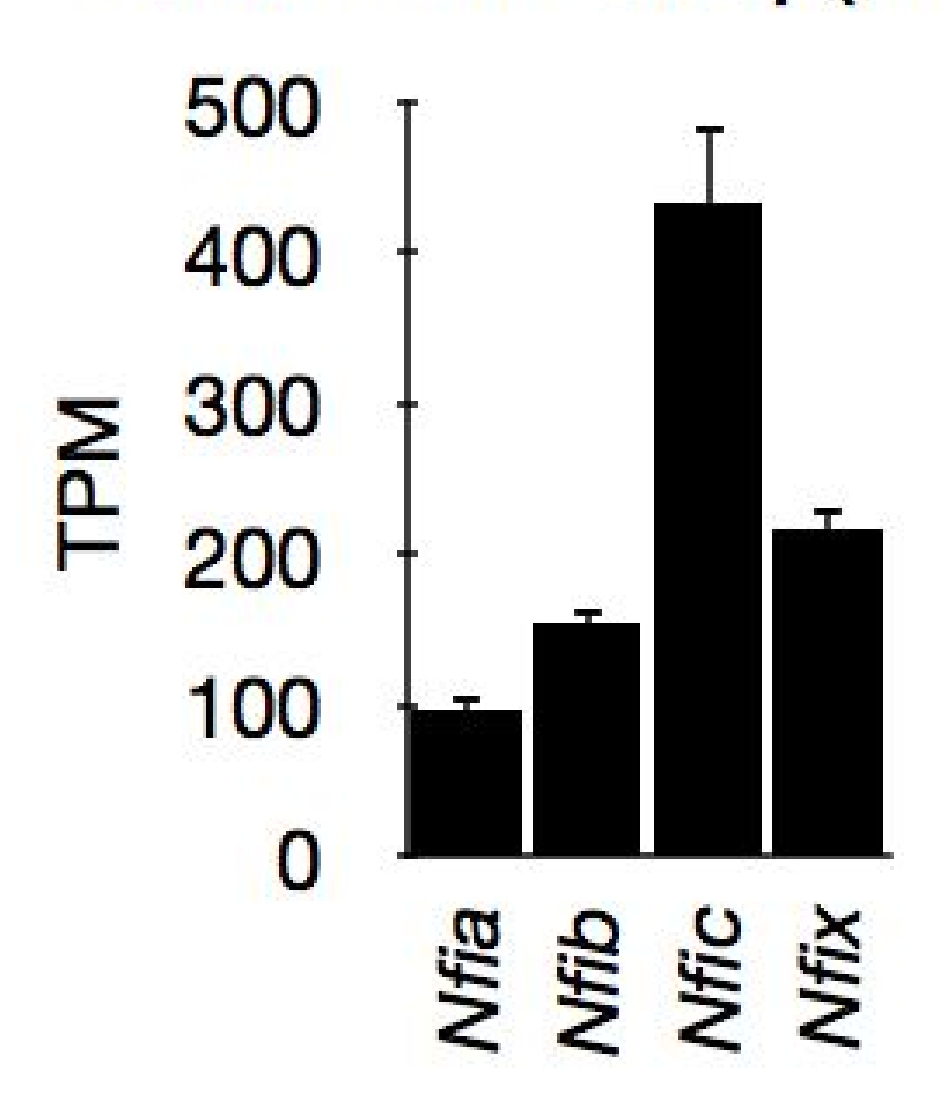

Bulk RNA-Seq (Human)

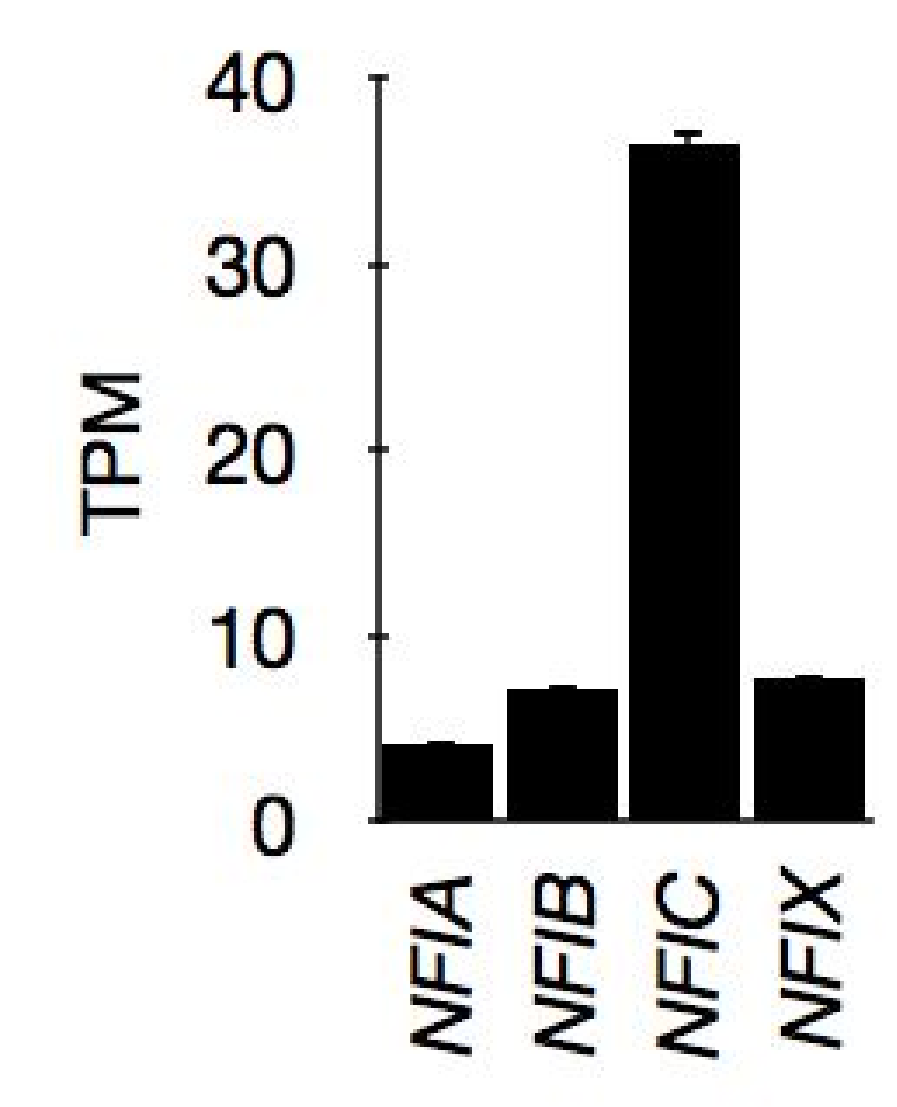

C
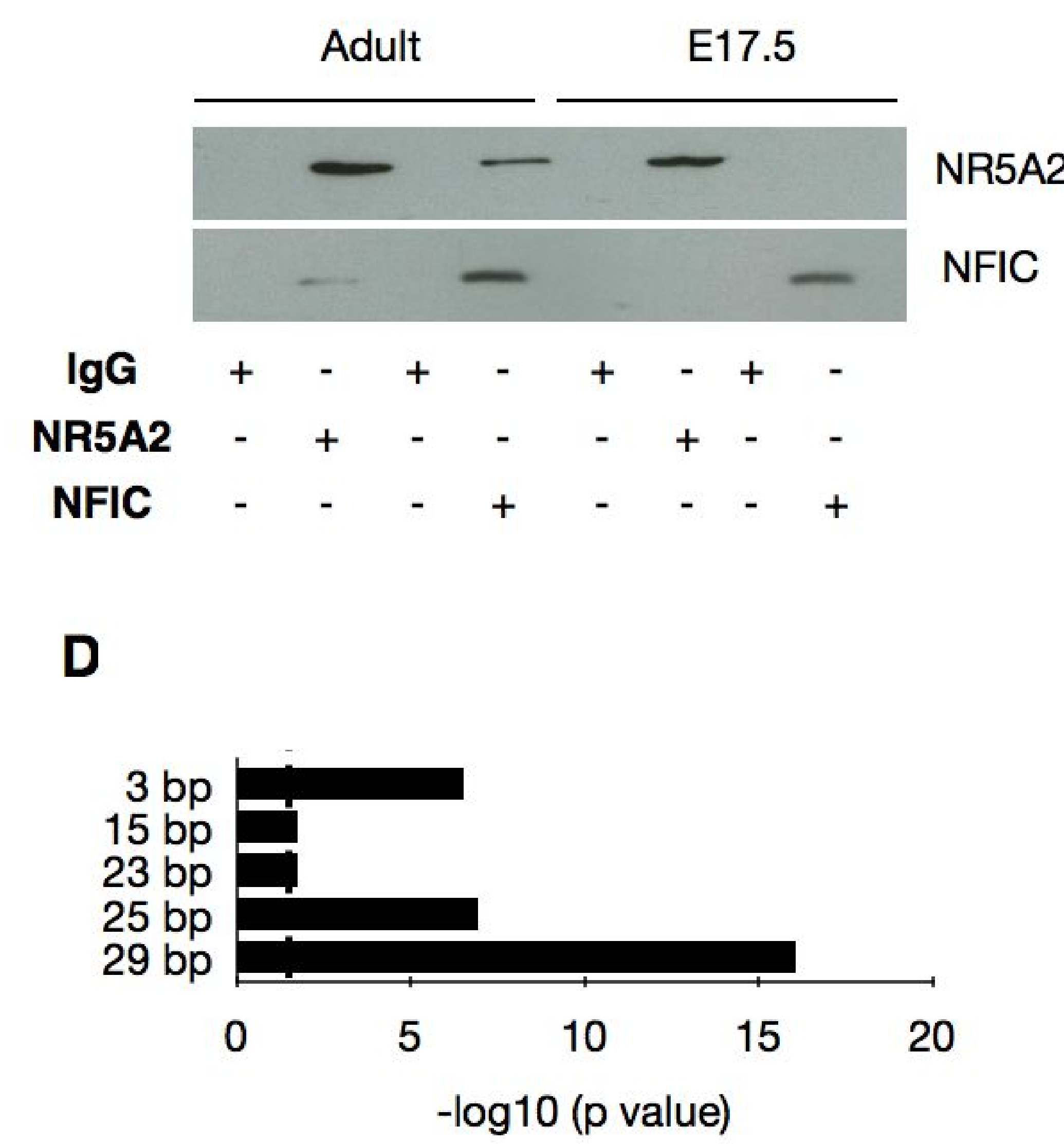

G

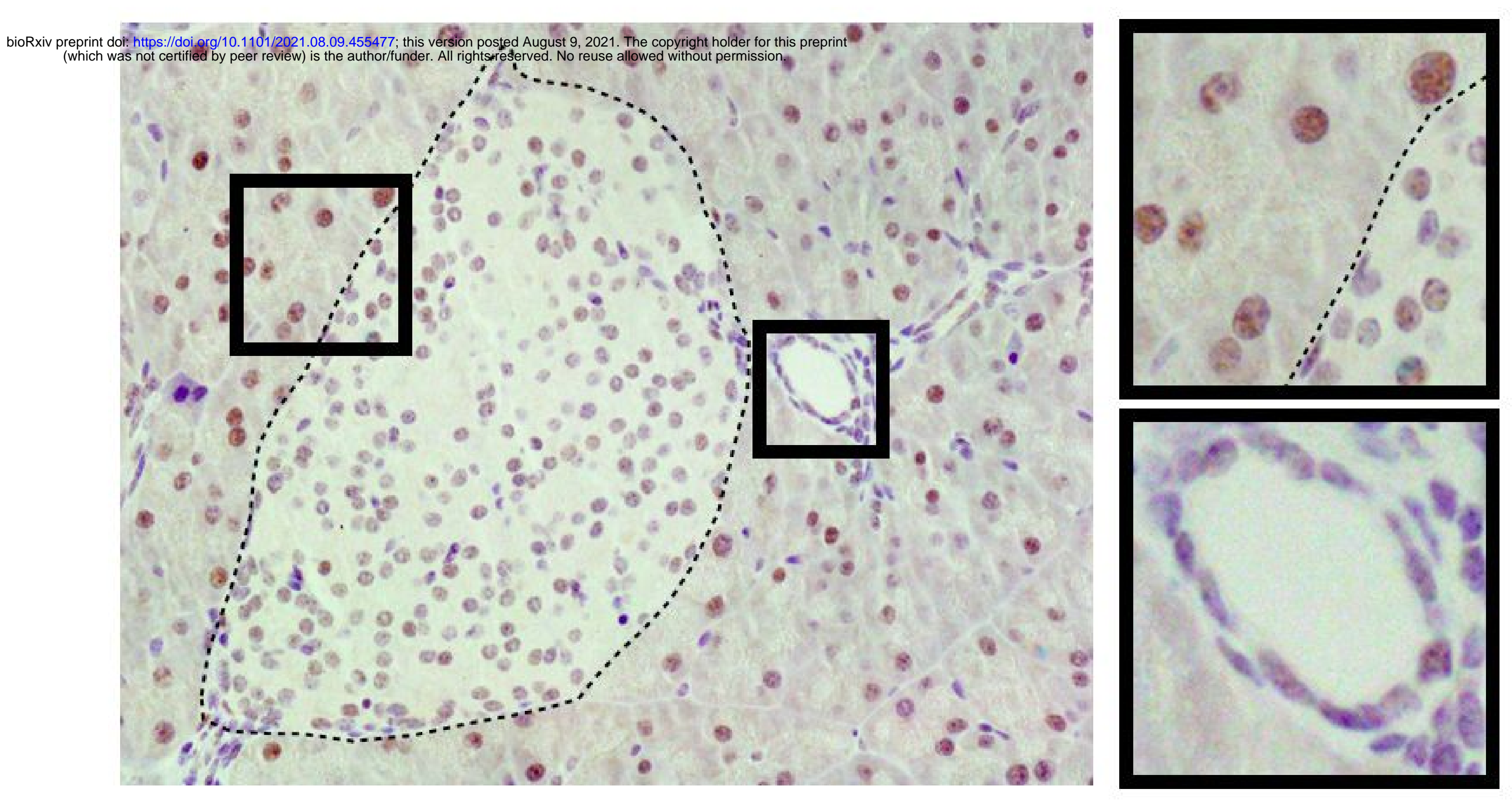

$\mathbf{F}$

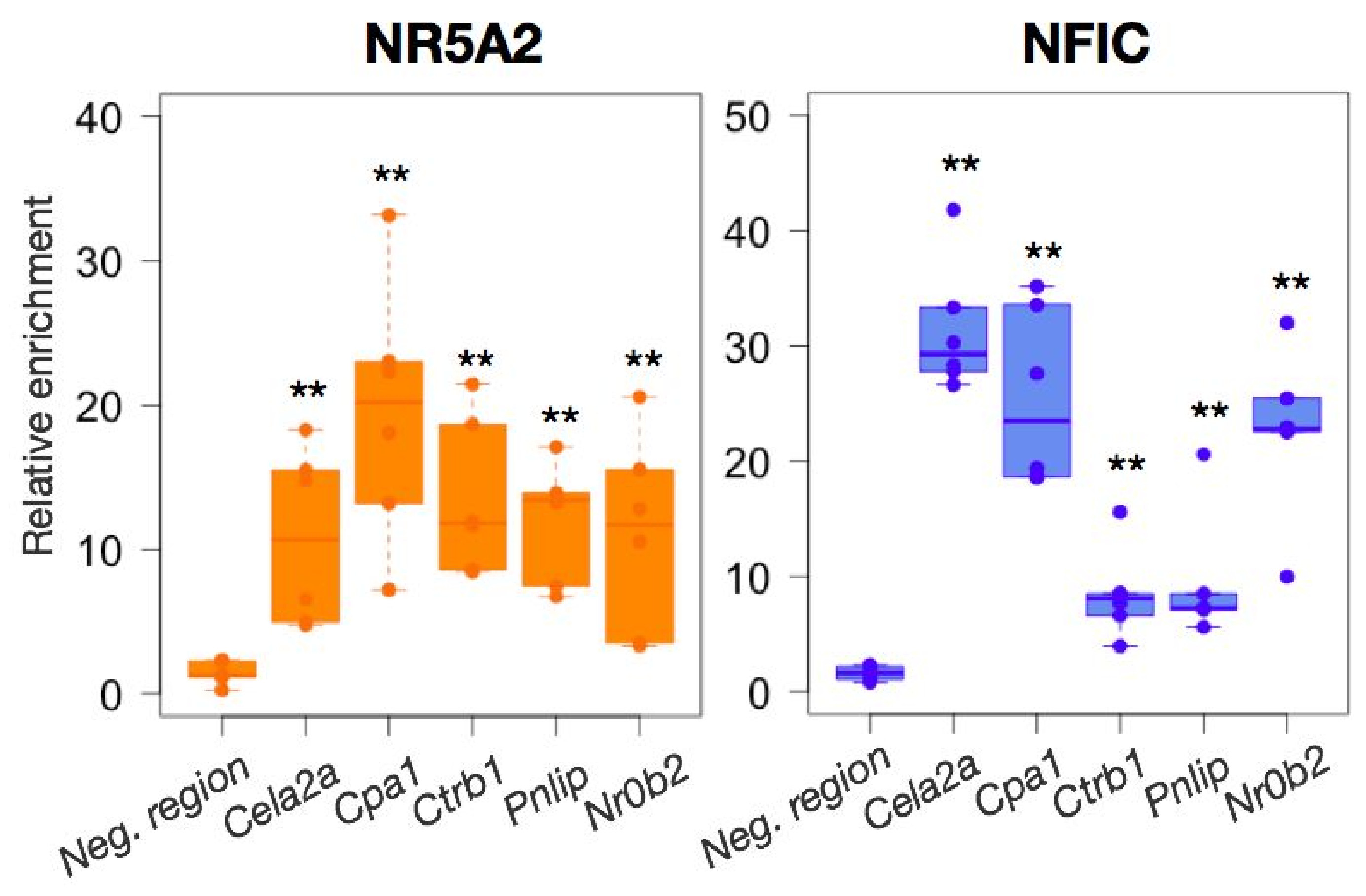

H

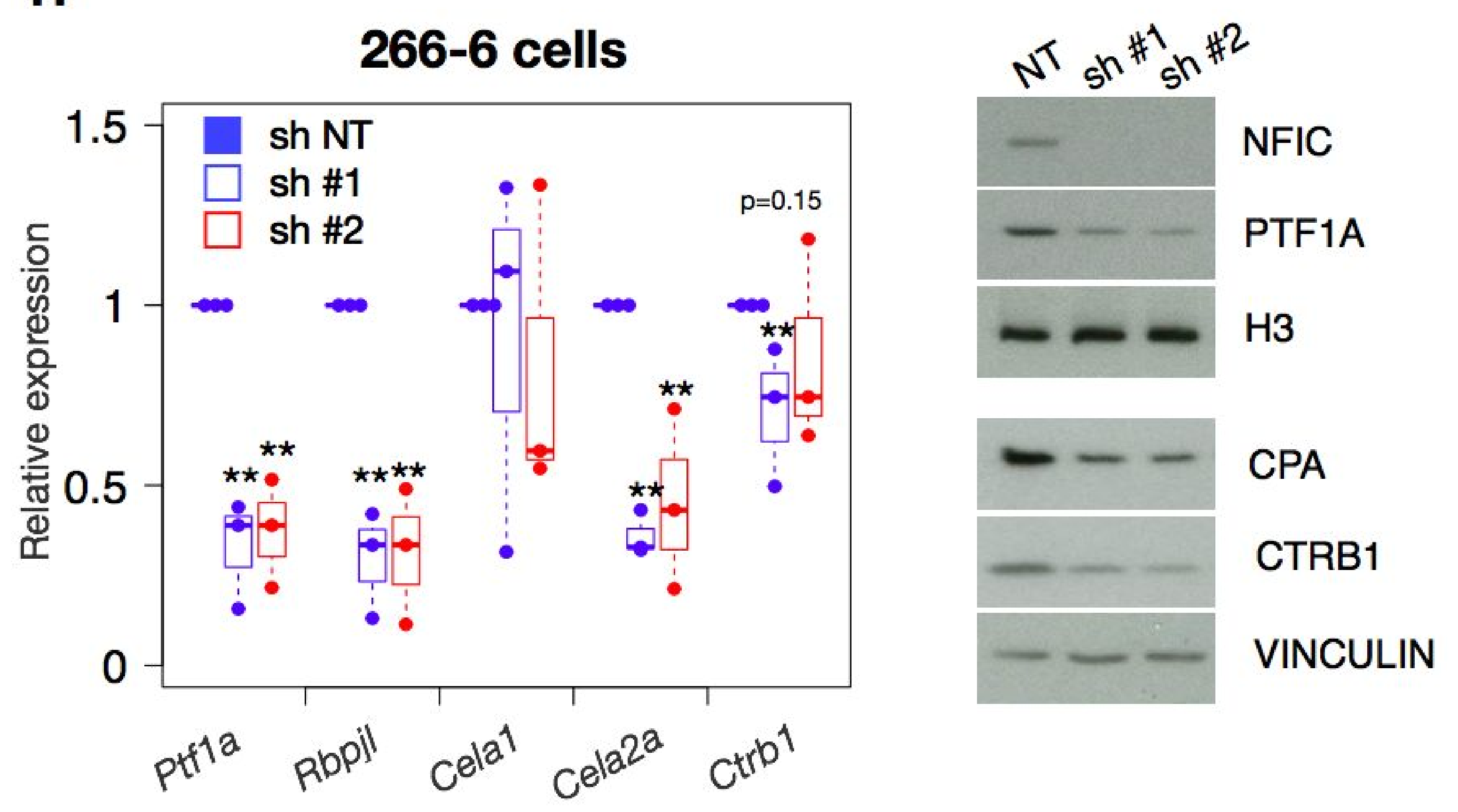




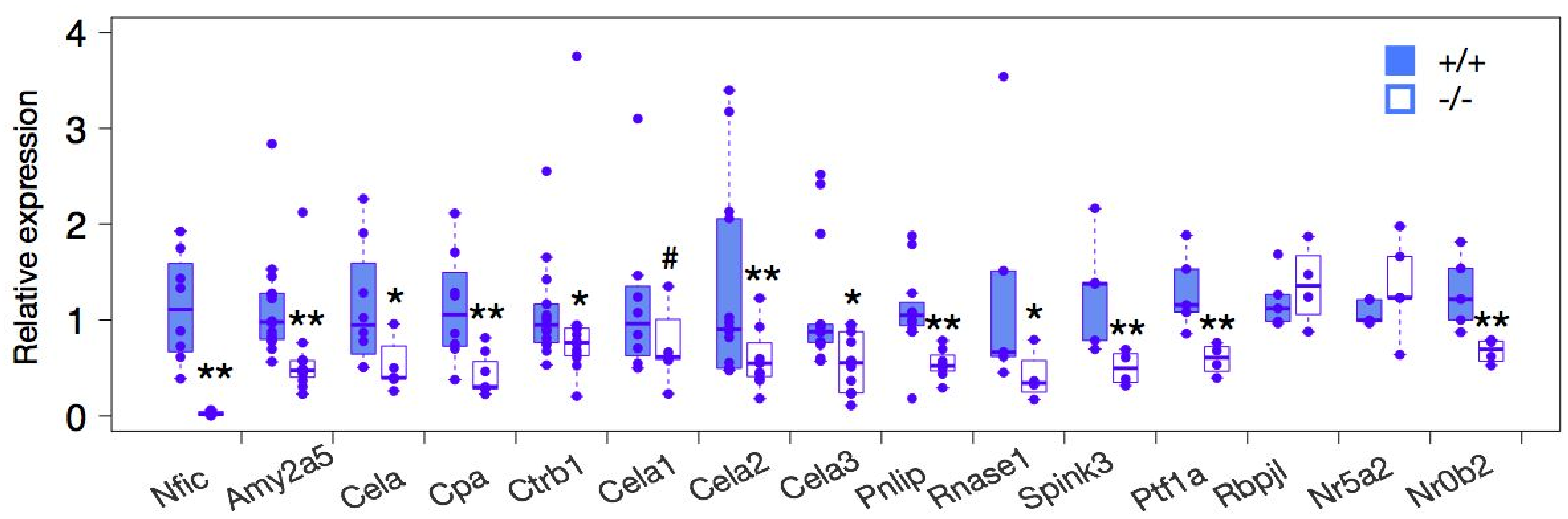

B

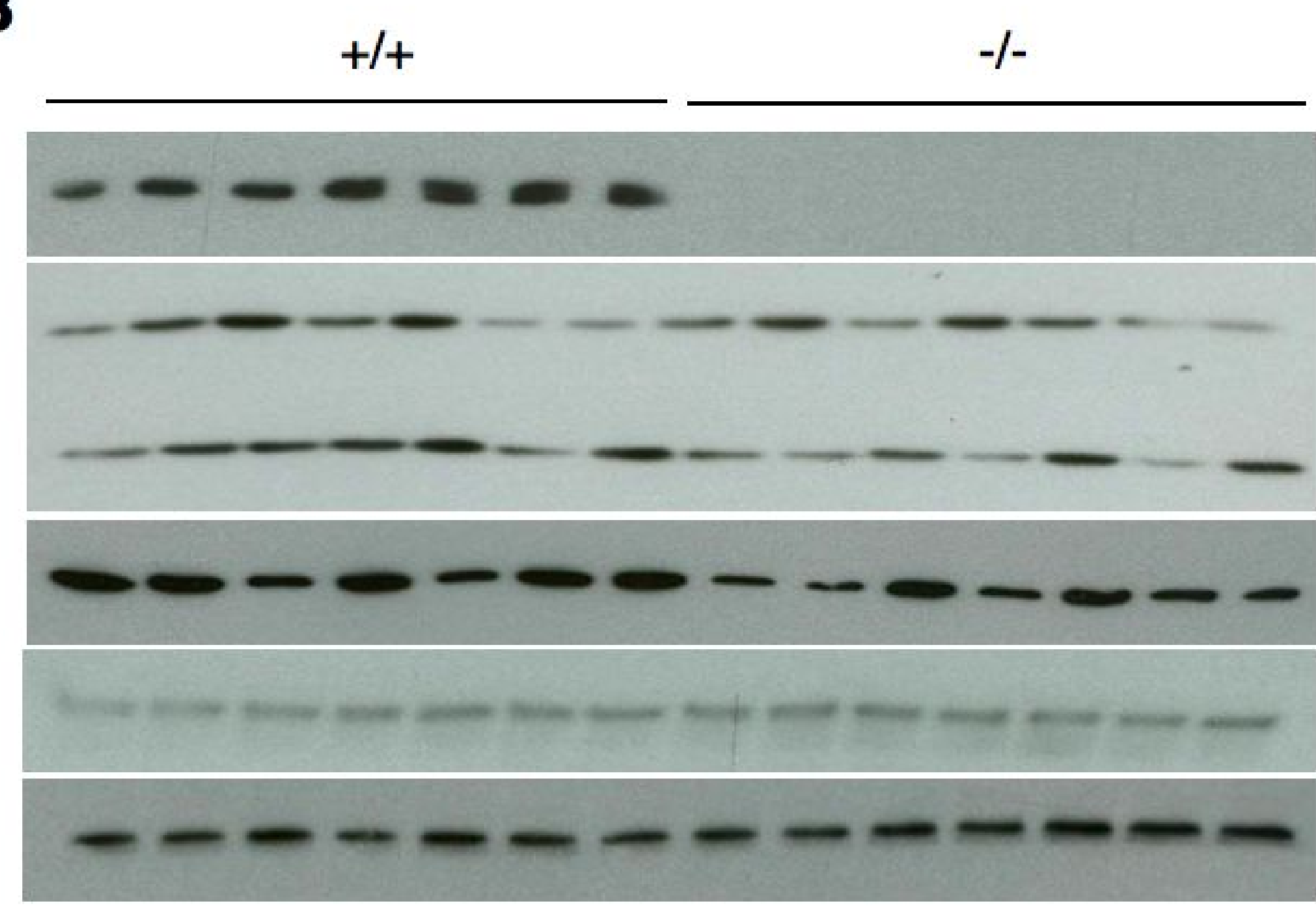

C

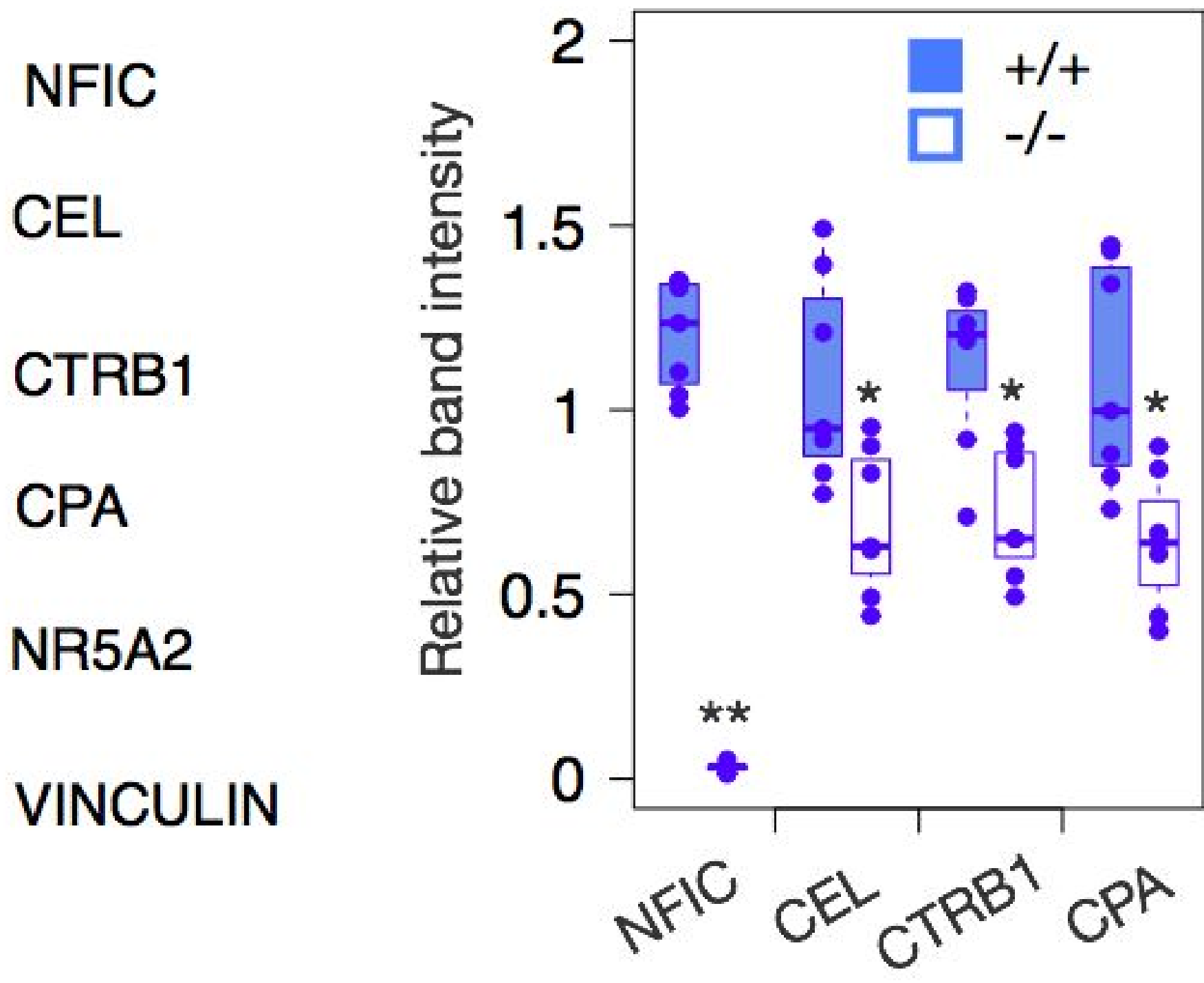

D
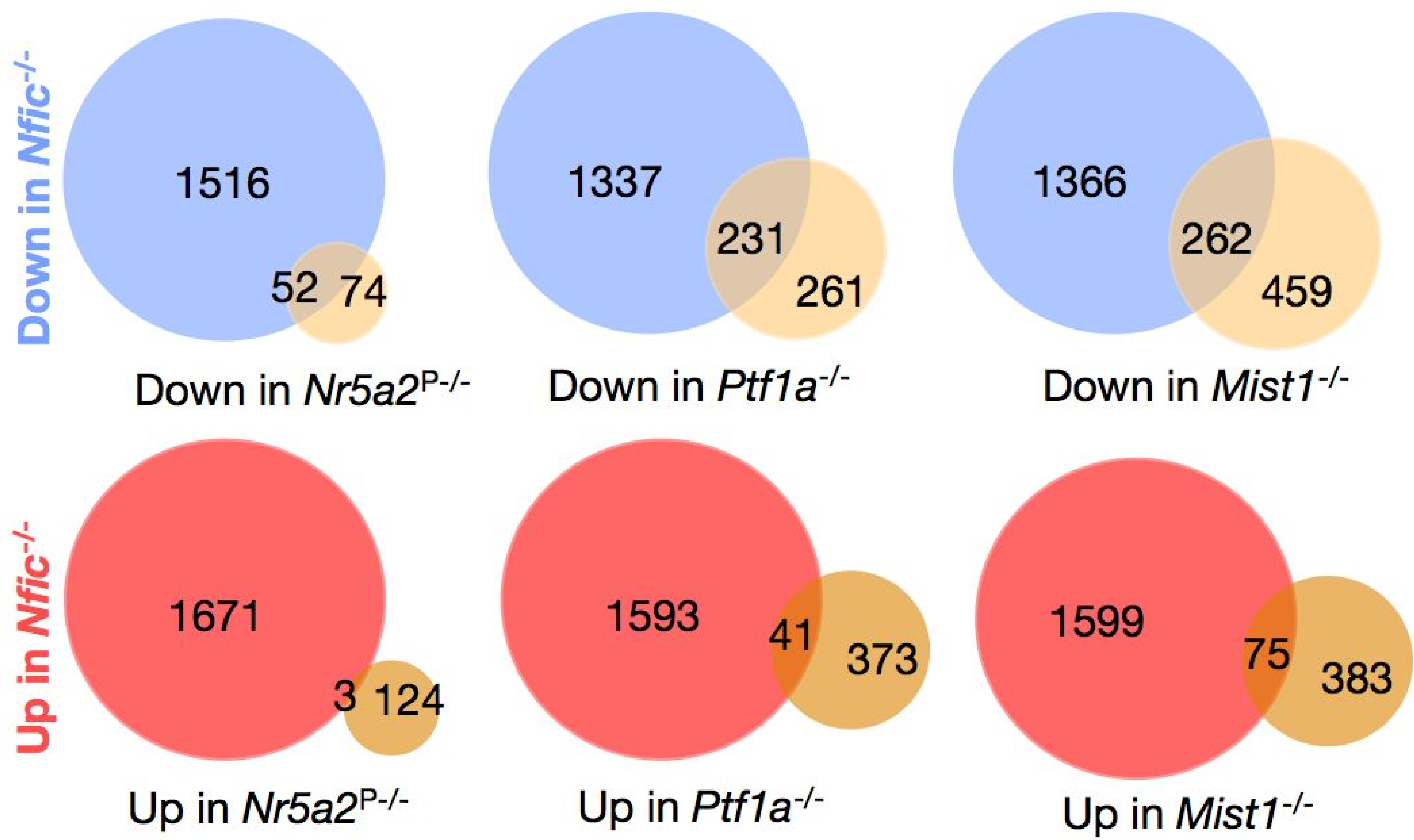

Up in $N r 5 a 2^{\mathrm{P}-/-}$

Up in Ptf1a ${ }^{-/-}$

Up in Mist1 ${ }^{-1-}$ 
A

\begin{tabular}{|c|c|c|c|c|}
\hline de novo motifs & $\begin{array}{l}\text { best } \\
\text { match }\end{array}$ & $\begin{array}{l}\% \\
\text { target }\end{array}$ & $\begin{array}{c}\% \\
\text { backg. }\end{array}$ & $\begin{array}{l}-\log _{10} \\
(\mathrm{FDR})\end{array}$ \\
\hline$C$ & NF1 & 42.81 & 11.99 & \\
\hline & ATOH1 & 27.72 & 16.56 & 31 \\
\hline & NFIX & 42.93 & 30.04 & 19 \\
\hline & FOXA1 & 14.04 & s.67 & 201 \\
\hline & BORIS & 5.87 & 1.80 & 175 \\
\hline & NF1-h & 23.03 & 14.64 & 147 \\
\hline & GATA6 & 9.79 & 4.99 & 115 \\
\hline & NR & 6.45 & 3.08 & \\
\hline & & & & \\
\hline
\end{tabular}

C

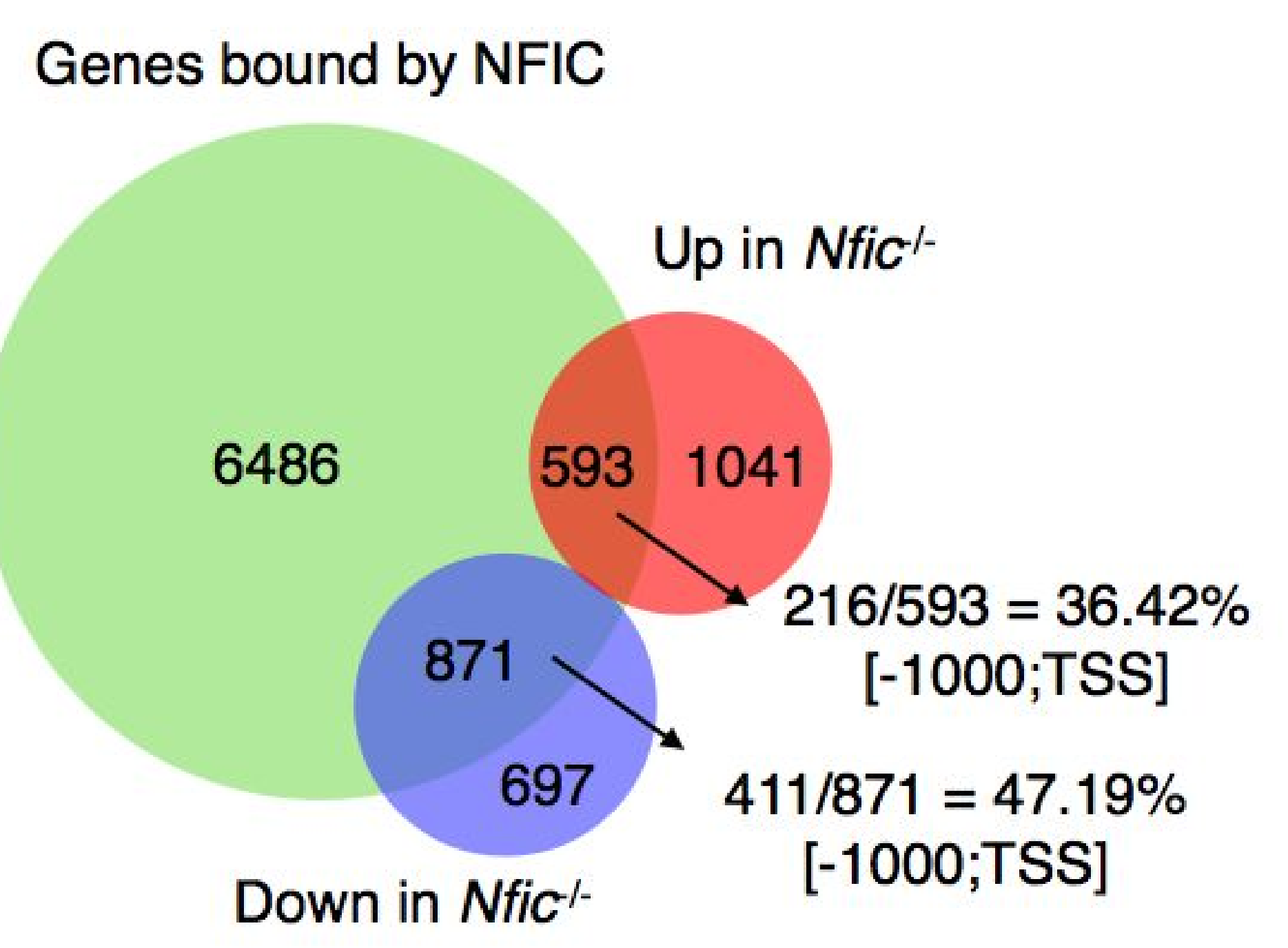

D

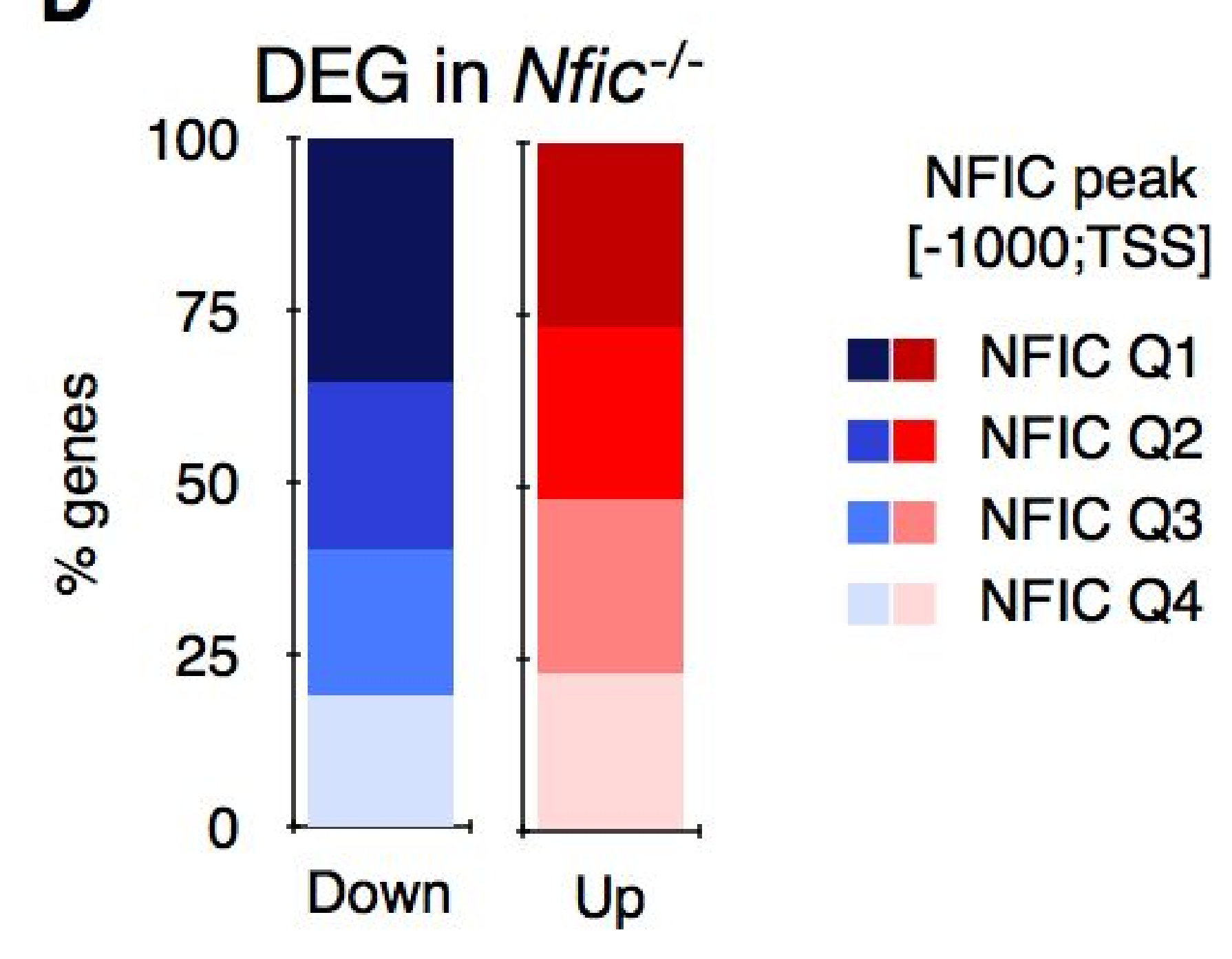

I

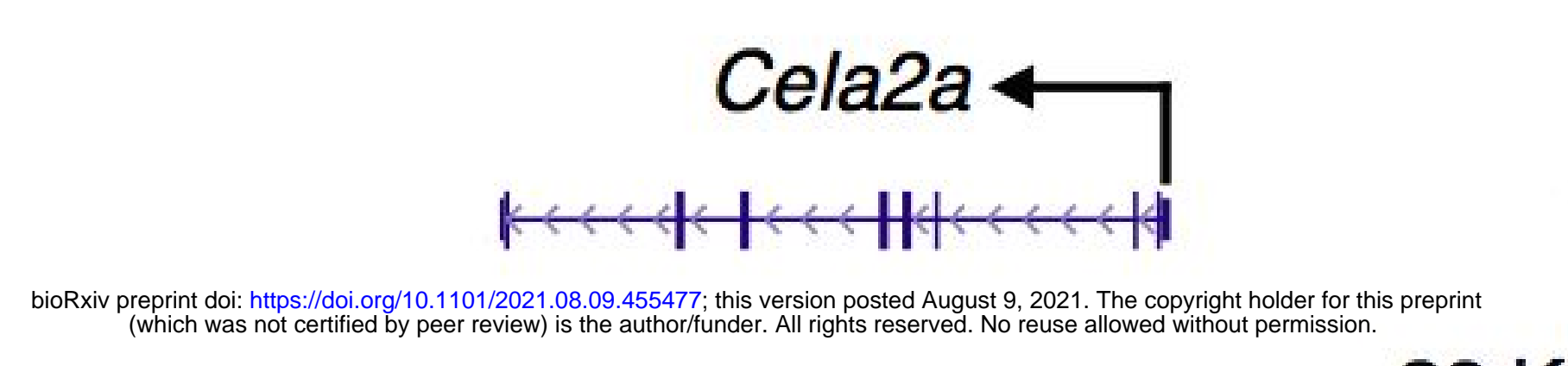

$20 \mathrm{~Kb}$

Input
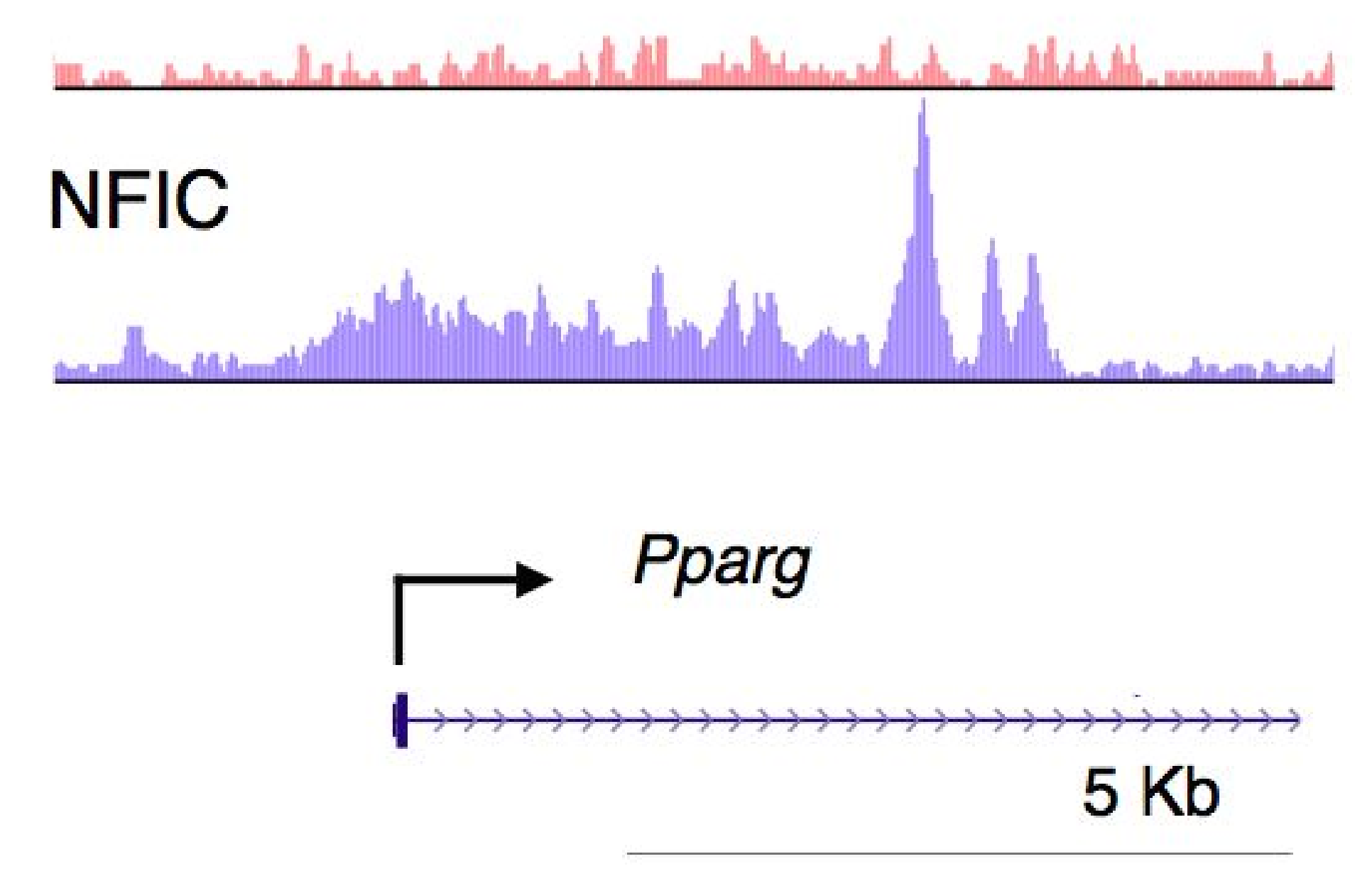

G

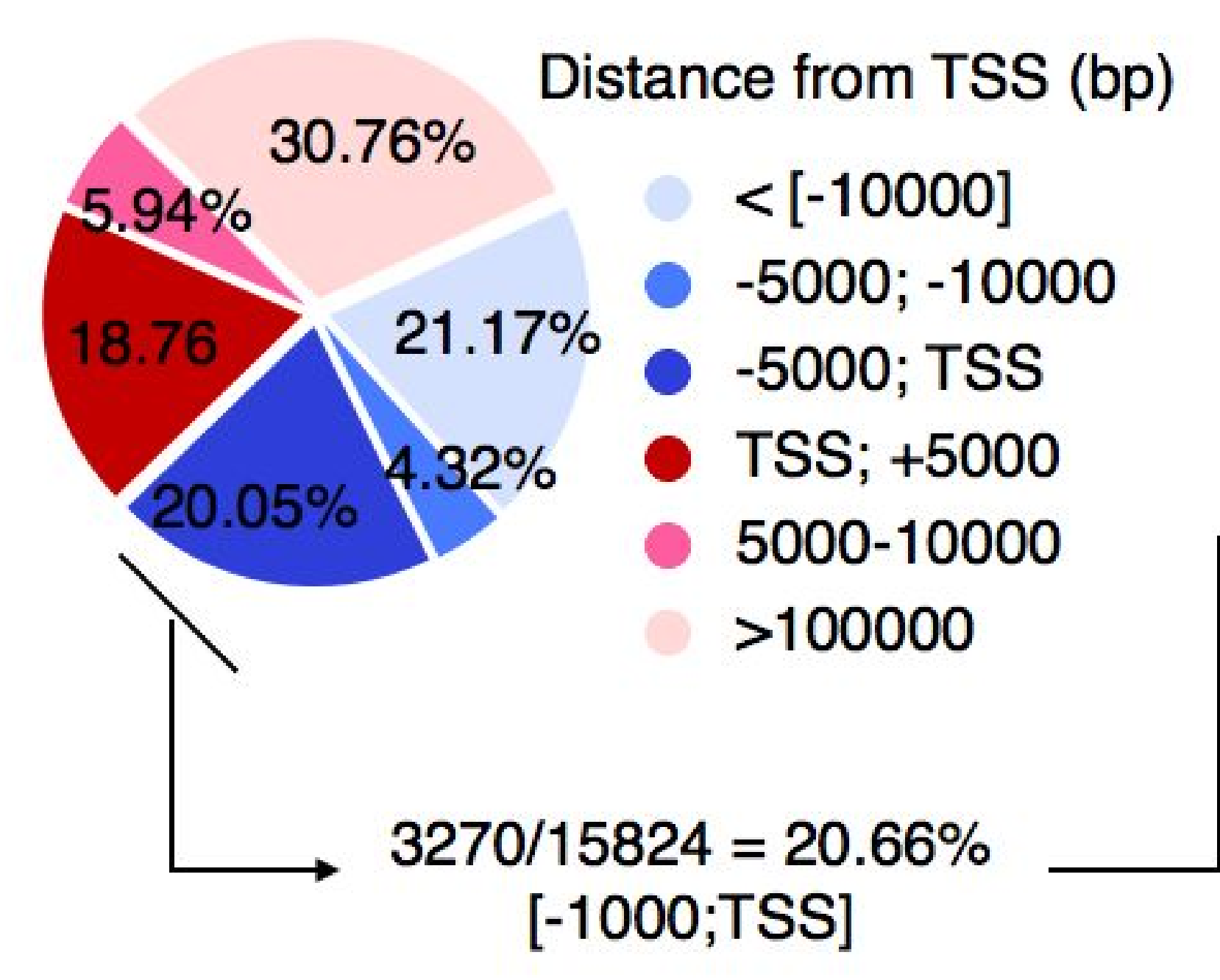

E

Peaks of genes bound by NFIC and Down in Nfic'-

\begin{tabular}{|c|c|c|c|c|}
\hline de novo motifs & $\begin{array}{c}\text { best } \\
\text { match }\end{array}$ & $\begin{array}{l}\% \\
\text { target }\end{array}$ & $\begin{array}{c}\% \\
\text { backg. }\end{array}$ & $\begin{array}{l}-\log _{10} \\
(\mathrm{FDR}\end{array}$ \\
\hline CCACSDTCMA & NF1 & 37.22 & 9.94 & \\
\hline & NFIX & 32.96 & 17.41 & \\
\hline & ZNF189 & 16.92 & 7.33 & \\
\hline & FOXM1 & 11.67 & 4.18 & \\
\hline & bHLH & 44.63 & 29.97 & \\
\hline & DUXA & 10.45 & 4.55 & \\
\hline & RBPJ & 13.61 & 7.16 & $21>>$ \\
\hline & SP2 & 9.57 & 4.48 & 10 \\
\hline & & & & \\
\hline
\end{tabular}

Down in $\mathrm{Nfic}^{- \text {- }}$ with NFIC peak $(\mathrm{n}=871)$
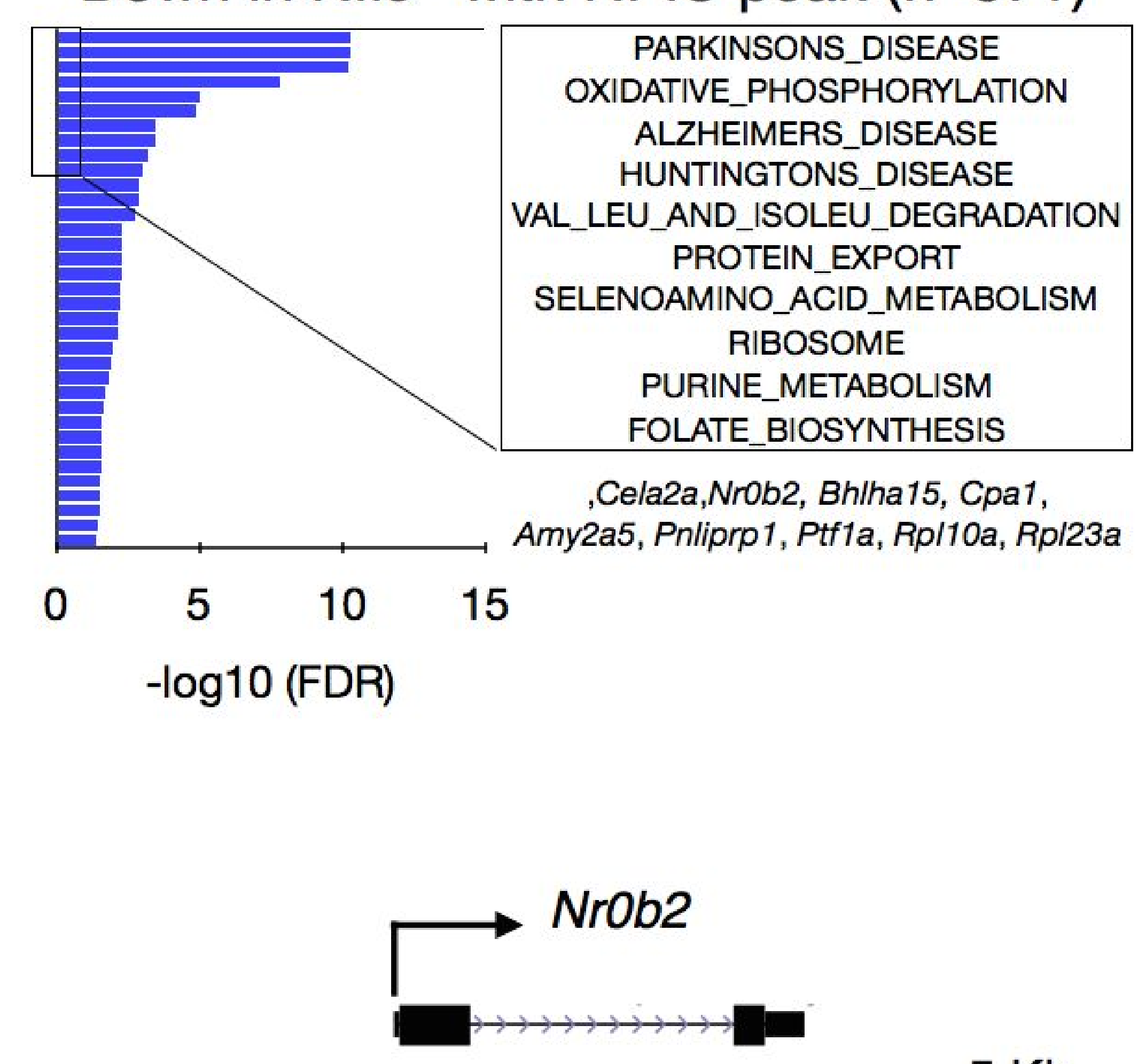

$5 \mathrm{~Kb}$
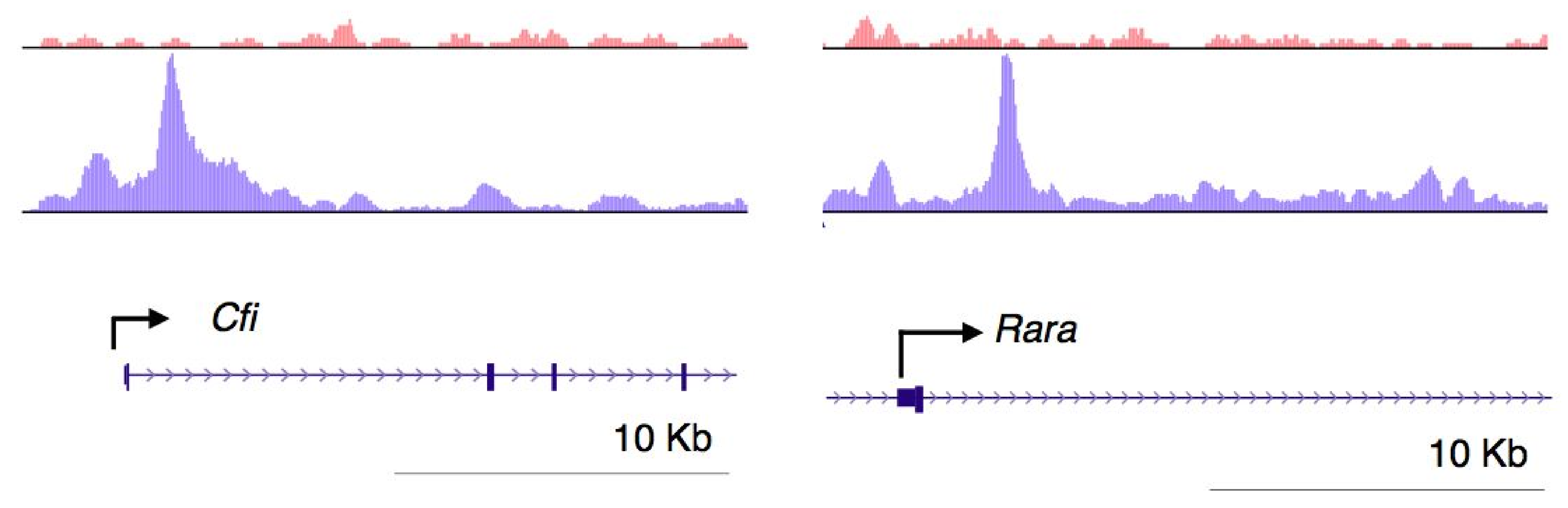

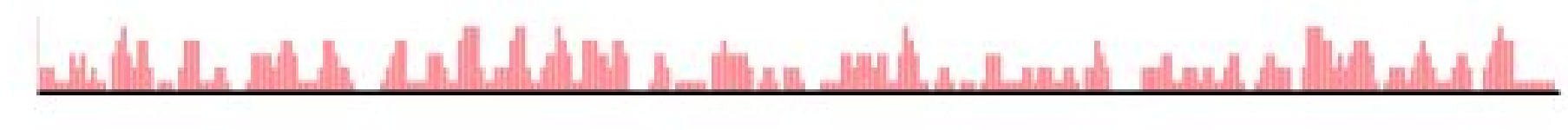

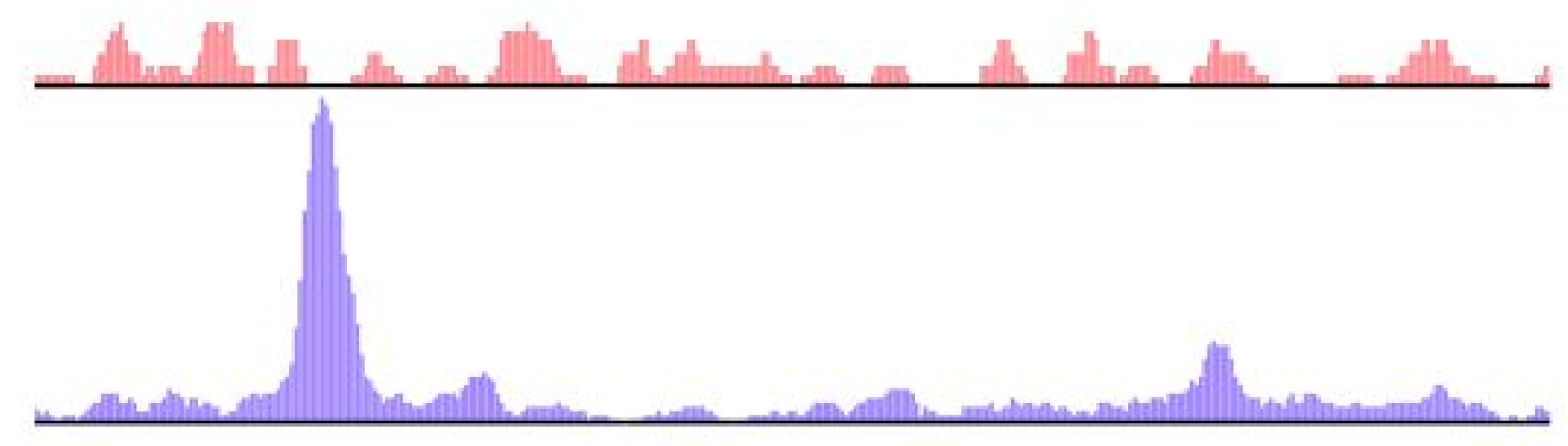

$\begin{array}{lcccc}\text { de novo motifs } & \text { best } & \% & \% & -\log _{10} \\ \text { match } & \text { target } & \text { backg } & \text { (FDR) }\end{array}$

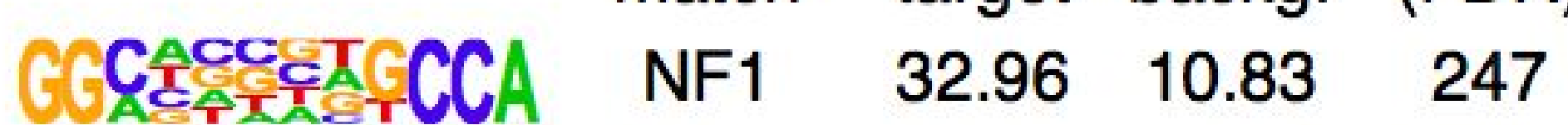

$\begin{array}{lllll}\text { AGCCGGGECC } & \text { SP2 } & 39.66 & 18.49 & 171\end{array}$

$\begin{array}{lllll}\text { EATTGCET } & \text { NFY } & 39.50 & 21.86 & 112\end{array}$

$\begin{array}{lllll}\text { 술CCGCAAGTG } & \text { ELK1 } & 18.36 & 9.83 & 48\end{array}$

$\begin{array}{llll}\text { IGGGAATTGTAG THAP } & 2.82 & 0.64 & 29\end{array}$

$\begin{array}{llll}\text { CCCIACGCGCCG CTCFL } & 3.81 & 1.15 & 28\end{array}$

$\begin{array}{lllll}\text { TGACCICATC } & \text { ATF1 } & 10.70 & 5.73 & 27\end{array}$

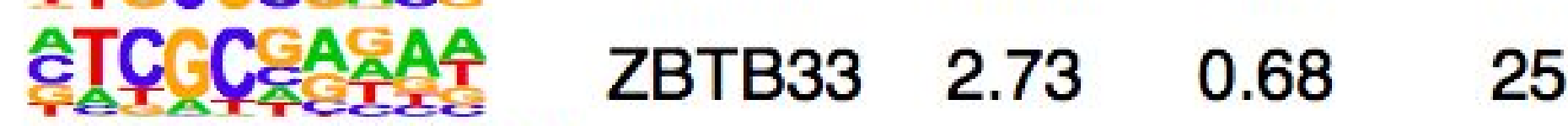

$\begin{array}{lllll}\text { CCGTCCCAGAAA } & \text { NFIX } & 7.84 & 3.87 & 24\end{array}$

\section{$\mathbf{F}$}

Peaks of genes bound by NFIC and Up in Nfic/-

\begin{tabular}{|c|c|c|c|c|}
\hline de novo motifs & $\begin{array}{l}\text { best } \\
\text { match }\end{array}$ & $\begin{array}{c}\% \\
\text { target }\end{array}$ & $\begin{array}{c}\% \\
\text { backg }\end{array}$ & $\begin{array}{l}-\log _{10} \\
(\mathrm{FDRR})\end{array}$ \\
\hline & $\mathrm{NF} 1$ & 45.99 & 11.18 & 171 \\
\hline$c$ & TLX?(NR) & 29.84 & 16.75 & 24 \\
\hline IEIIIAAC & HNF1 & 8.32 & 2.82 & 17 \\
\hline CAAGA & NFIX & 26.03 & 16.06 & \\
\hline CCAGAT TT & TCF3 & 0.59 & $<0.01$ & \\
\hline & CTCFL & 1.17 & 0.05 & \\
\hline
\end{tabular}

H

Up in $\mathrm{Nfic}^{- \text {- }}$ with NFIC peak $(\mathrm{n}=593)$
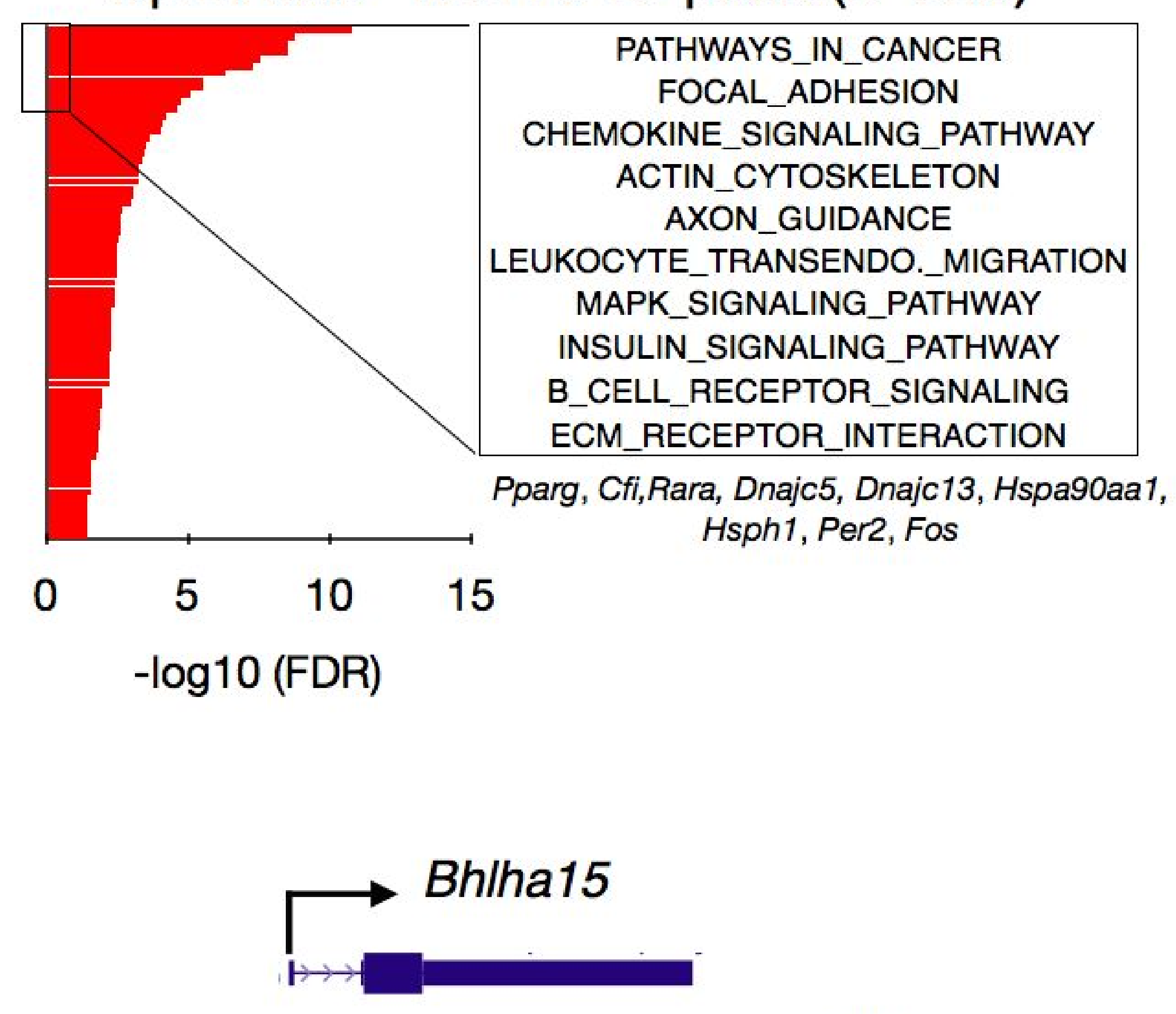

$5 \mathrm{~Kb}$

$10 \mathrm{~Kb}$ 
A

Merge 5.8 rRNA CDH1

$+/+$

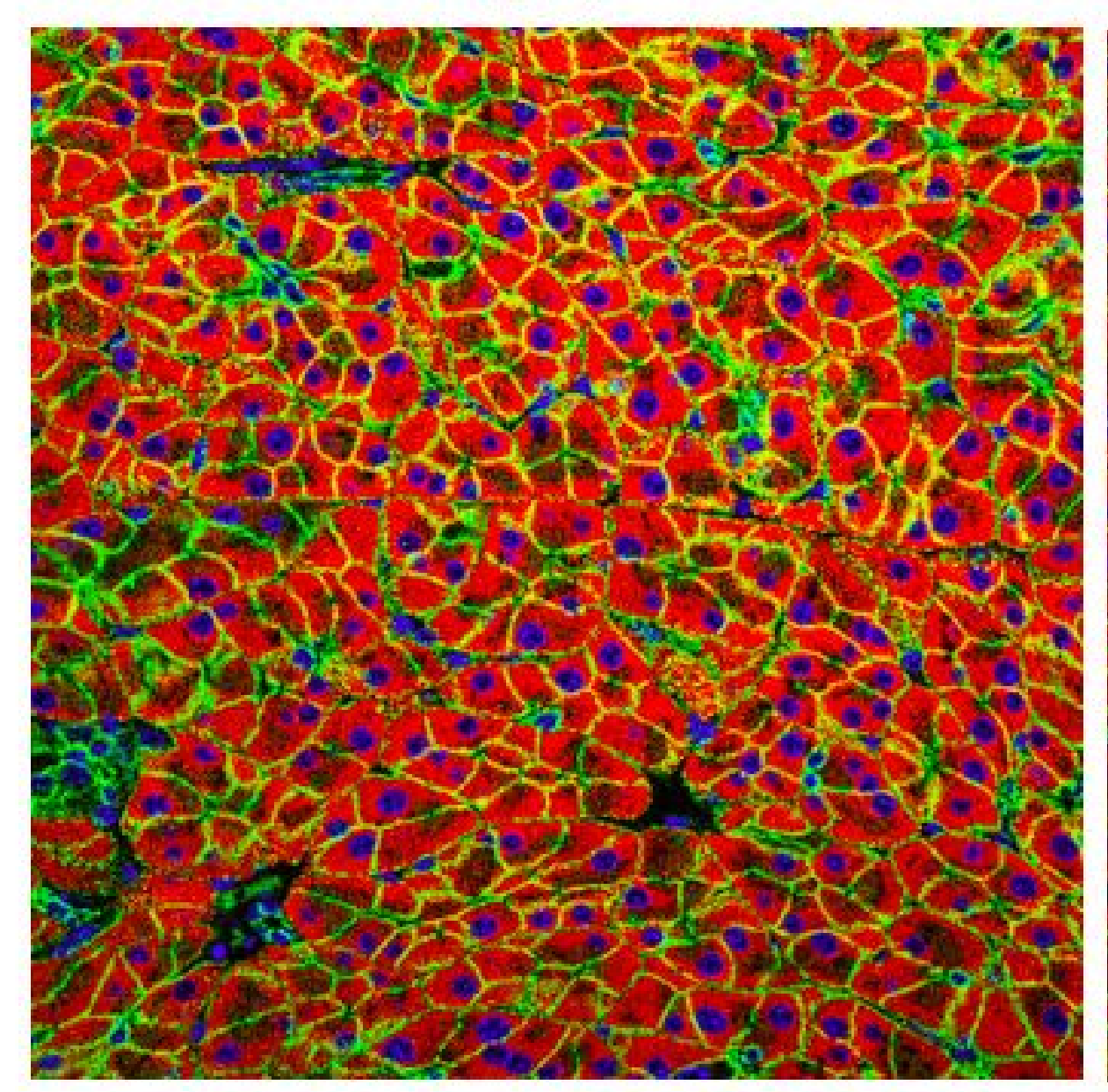

$-/-$

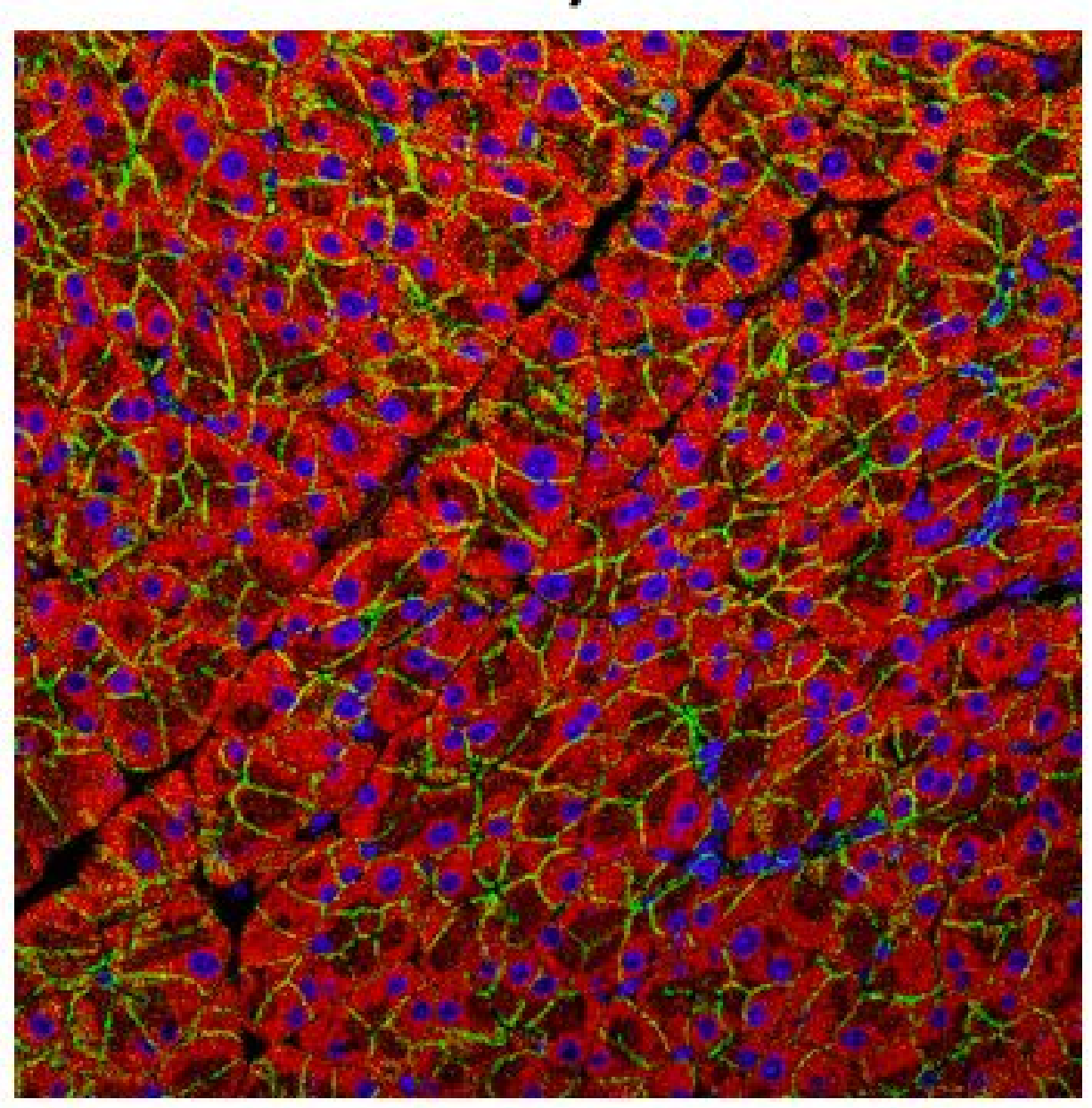

B

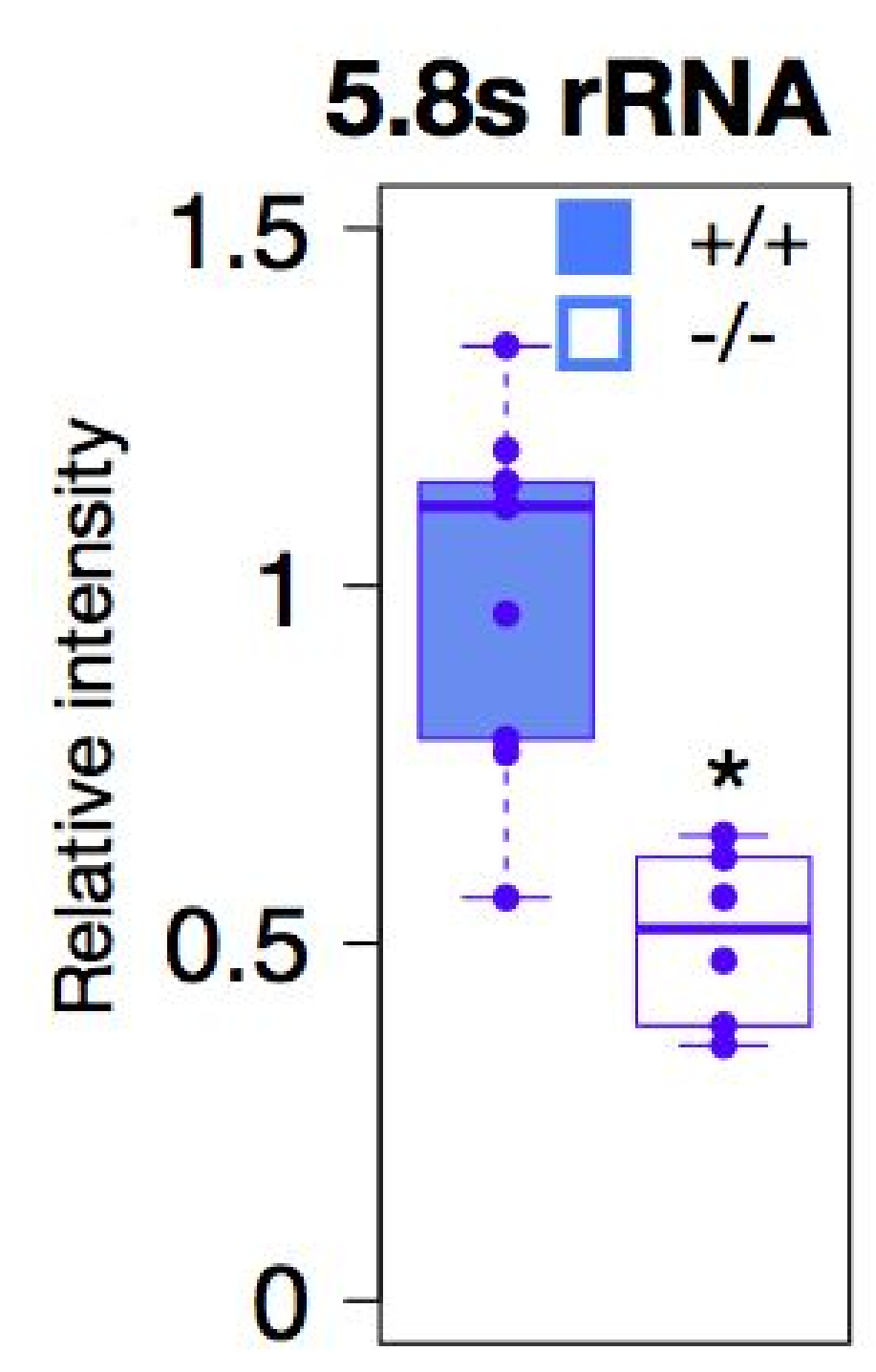

C

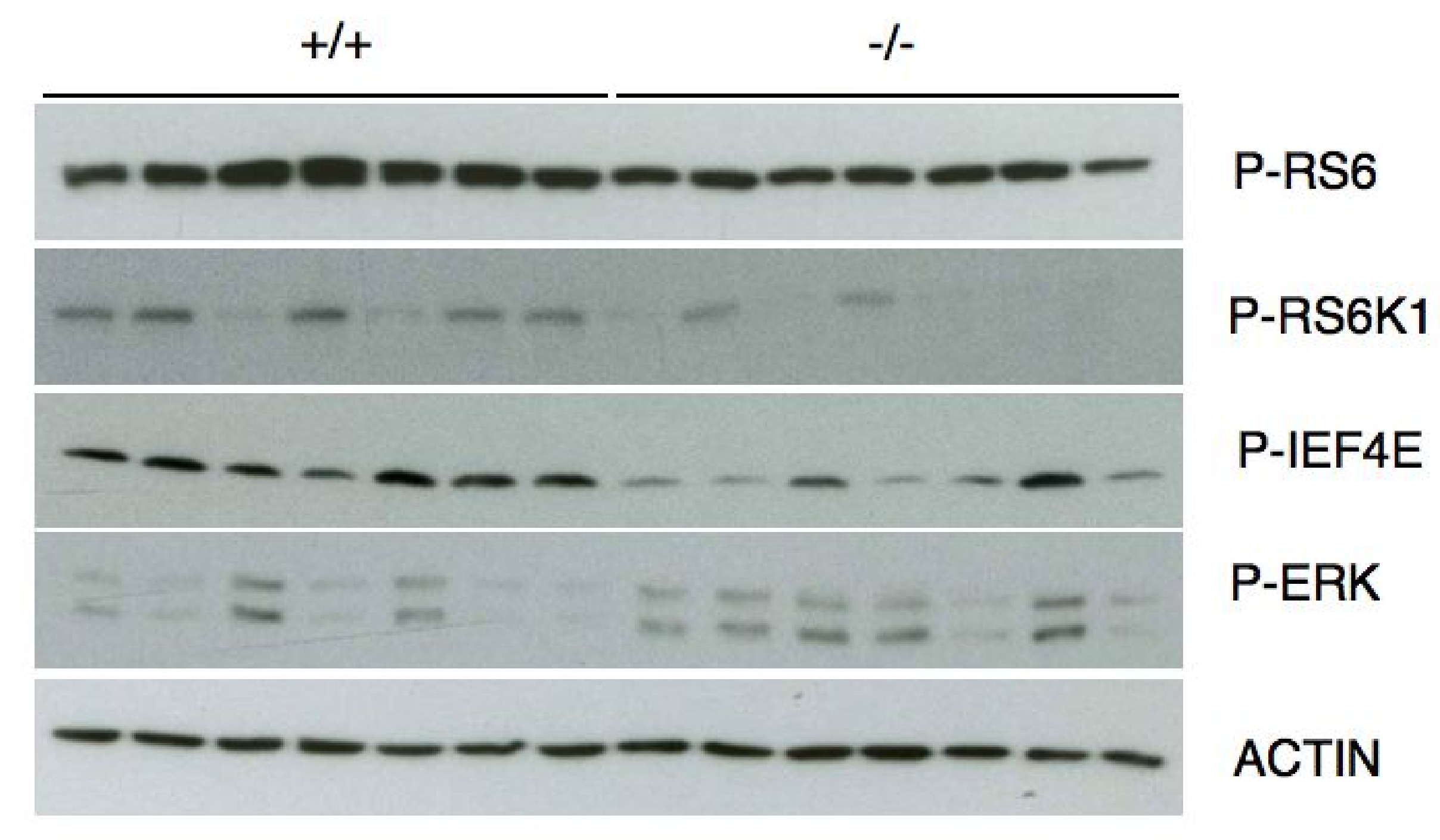

$\mathbf{F}$
D

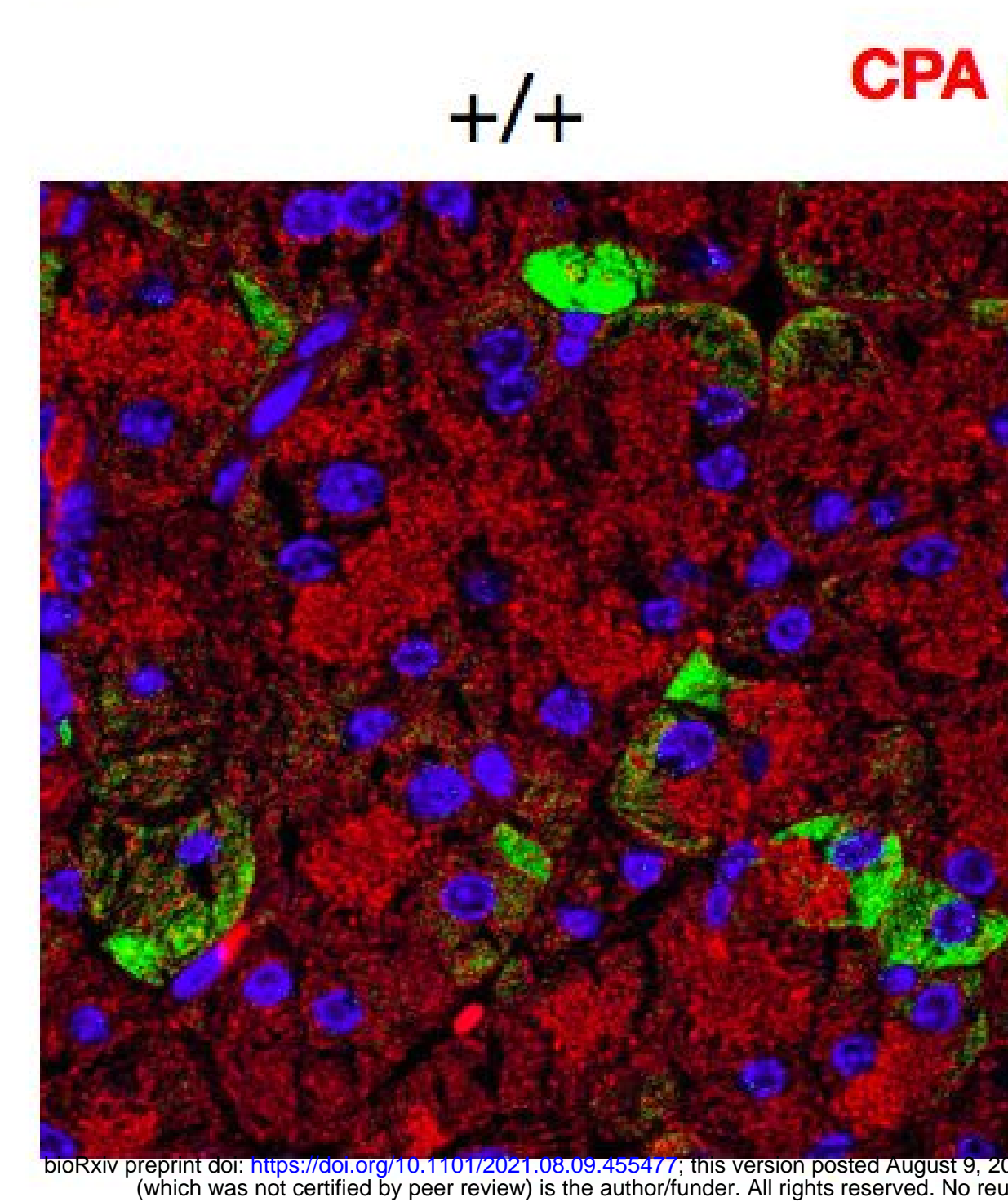

E

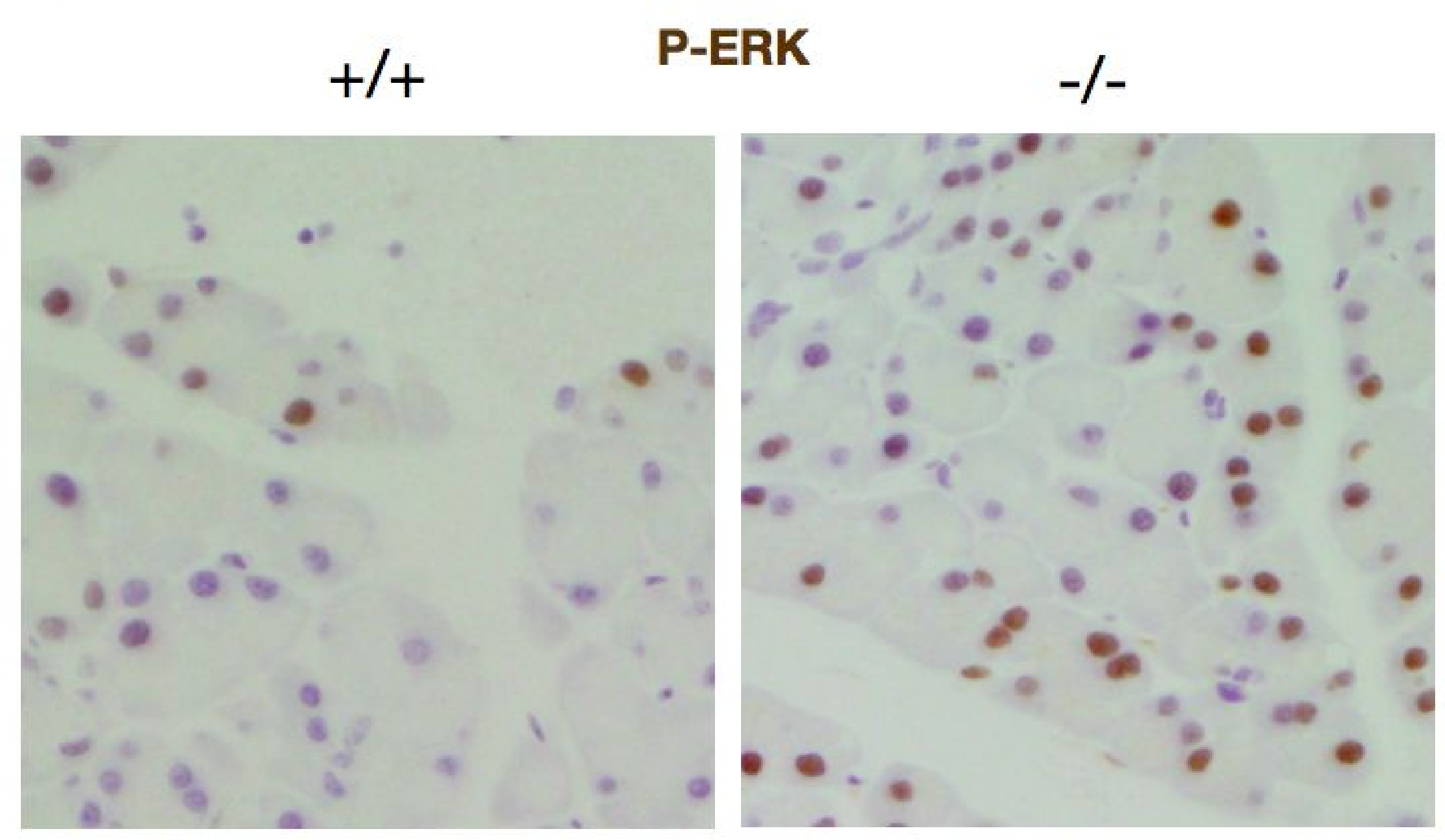

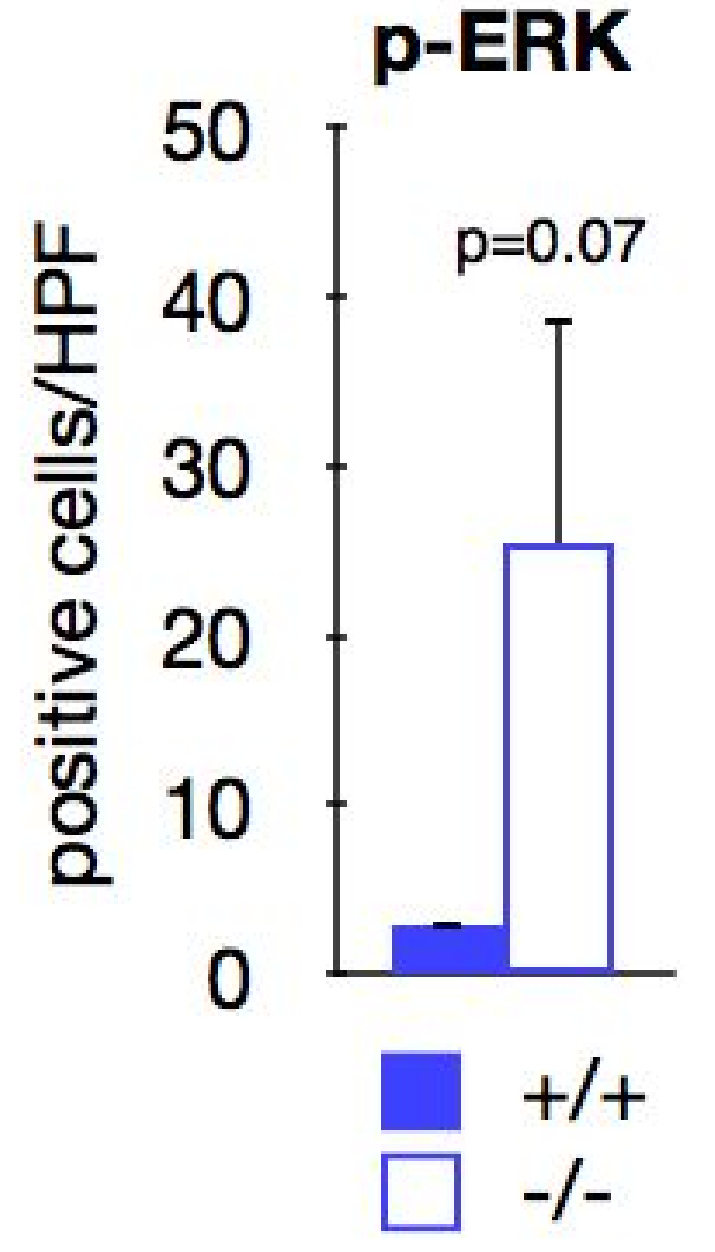

G

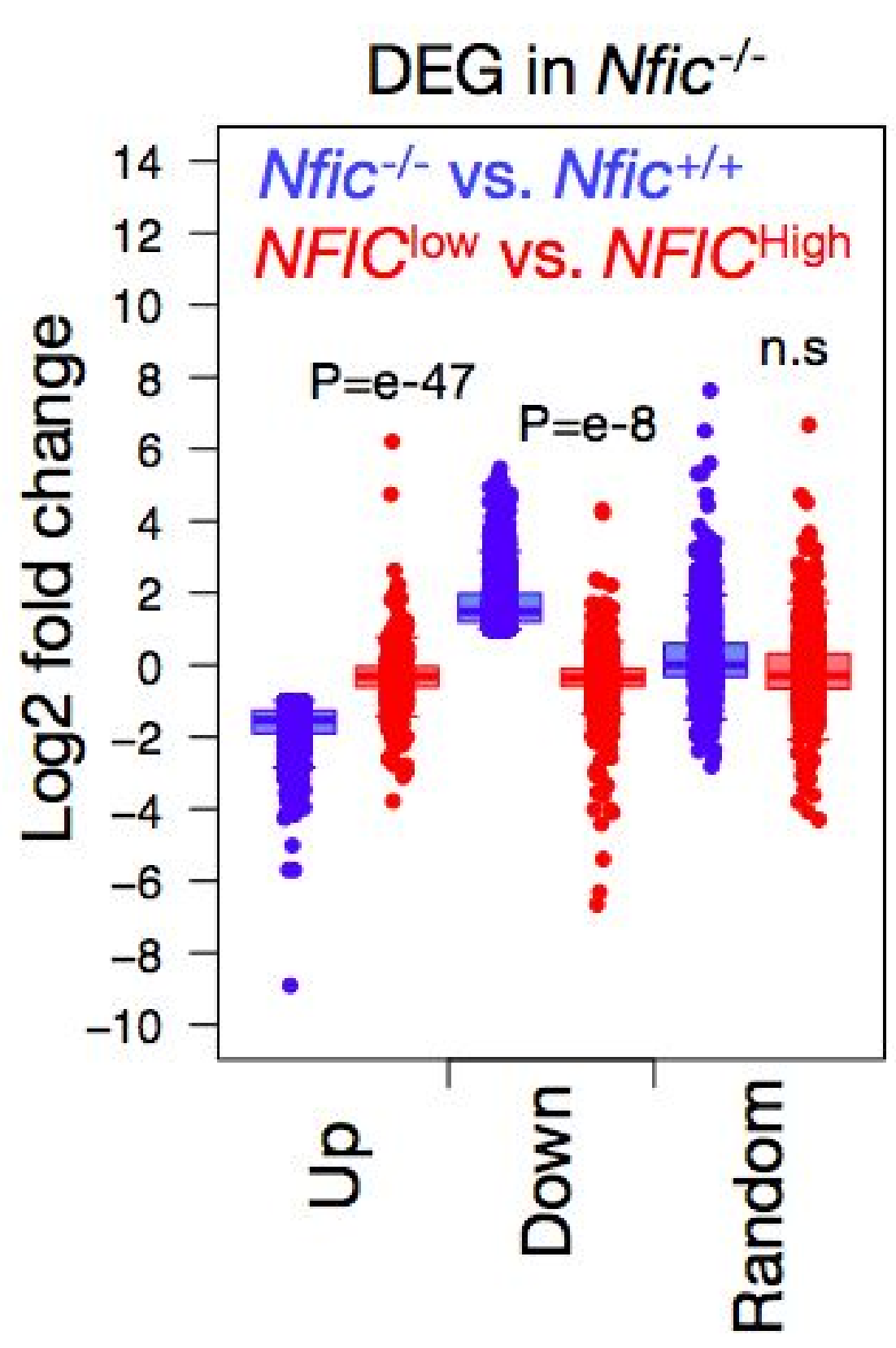

H

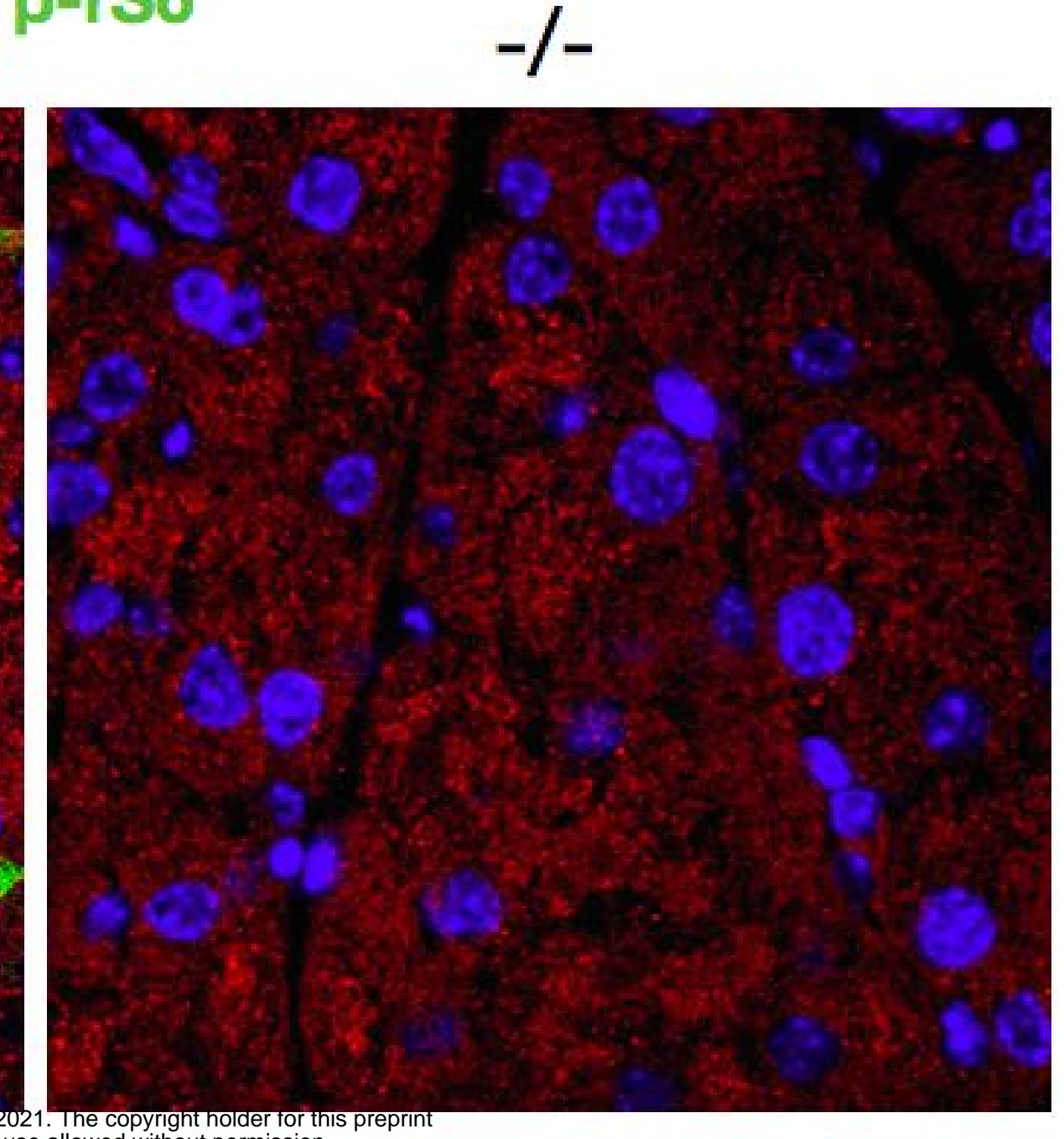

I

Gene Set Enrichment Analysis (KEGG)

Down-regulated in $\mathrm{NFIClow}$ vs. $\mathrm{NFIC}$ high and $\mathrm{Nfic}^{-/-}$vs $\mathrm{NfiC}^{+/+}$

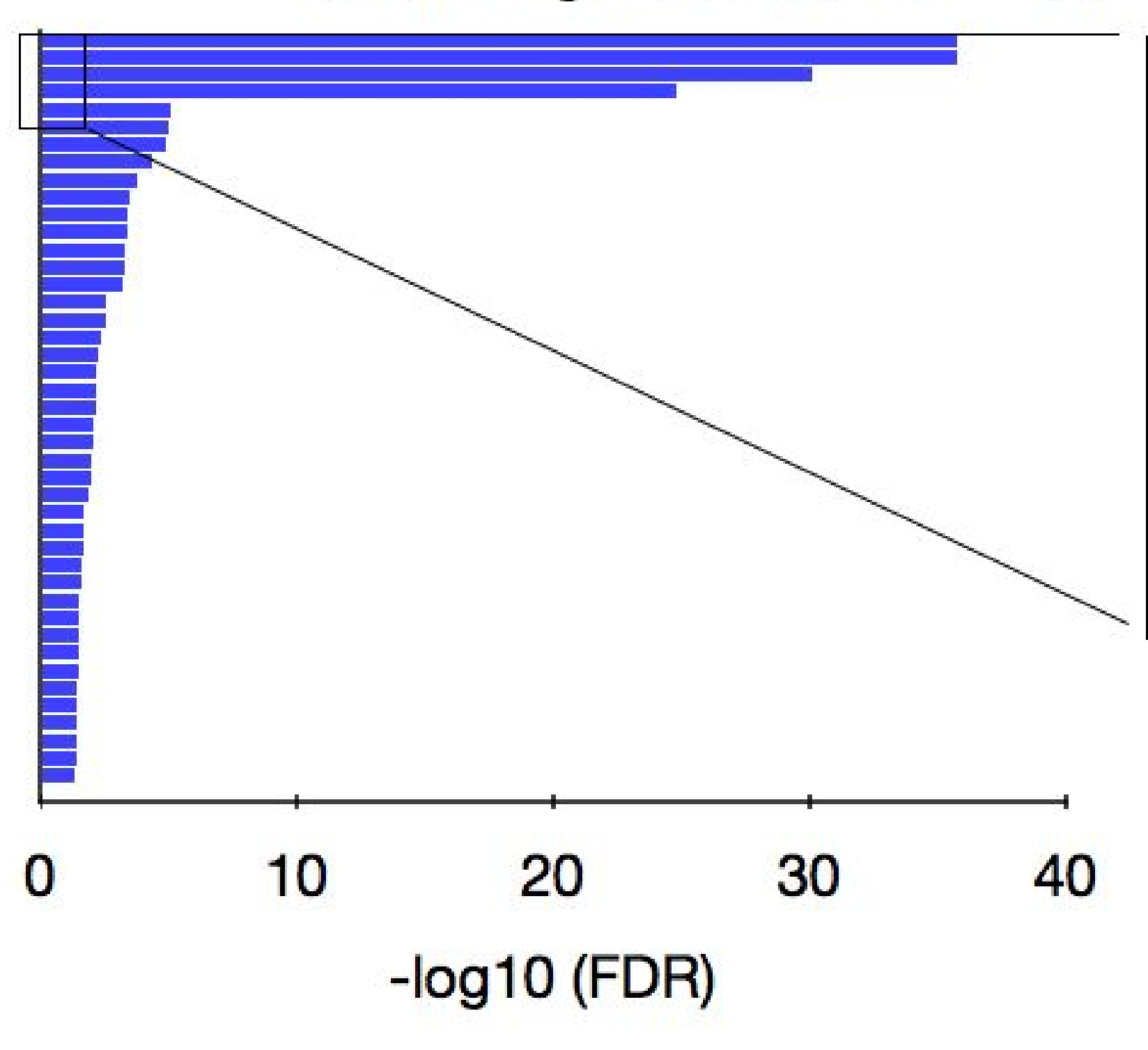

OXIDATIVE_PHOSPHORYLATION PARKINSONS_DISEASE HUNTINGTONS_DISEASE ALZHEIMERS_DISEASE RIBOSOME

CARDIAC_MUSCLE_CONTRACTION LYSOSOME

SPHINGOLIPID_METABOLISM PROTEASOME BLADDER_CANCER

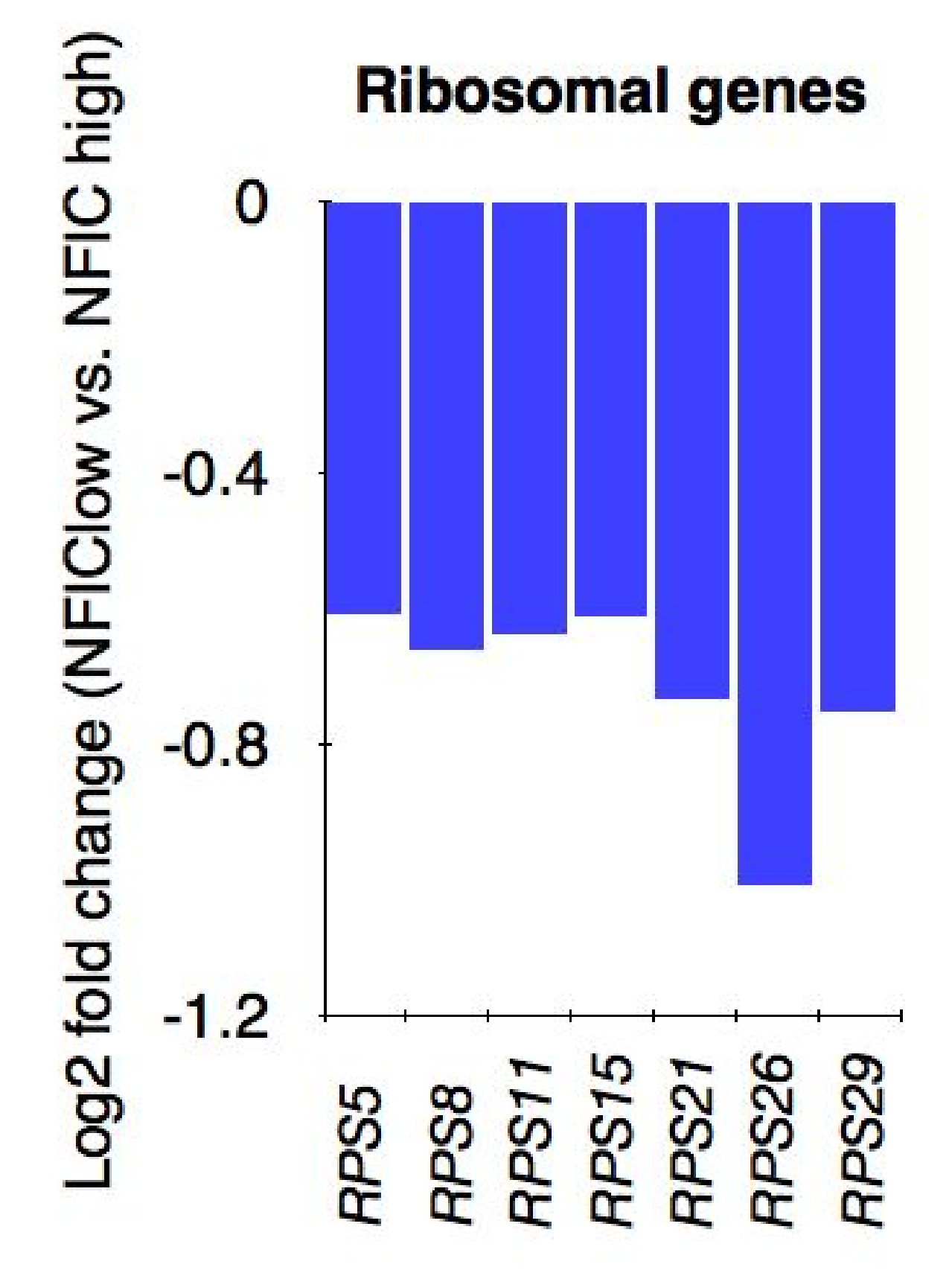


A

\section{Gene Set Enrichment Analysis}

AUTOPHAGY MTOR SIGNALING PROTEIN SECRETION

UPR

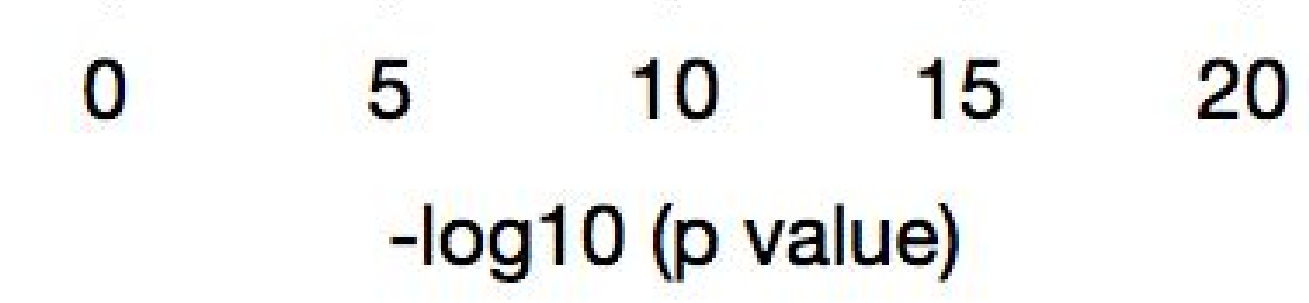

C

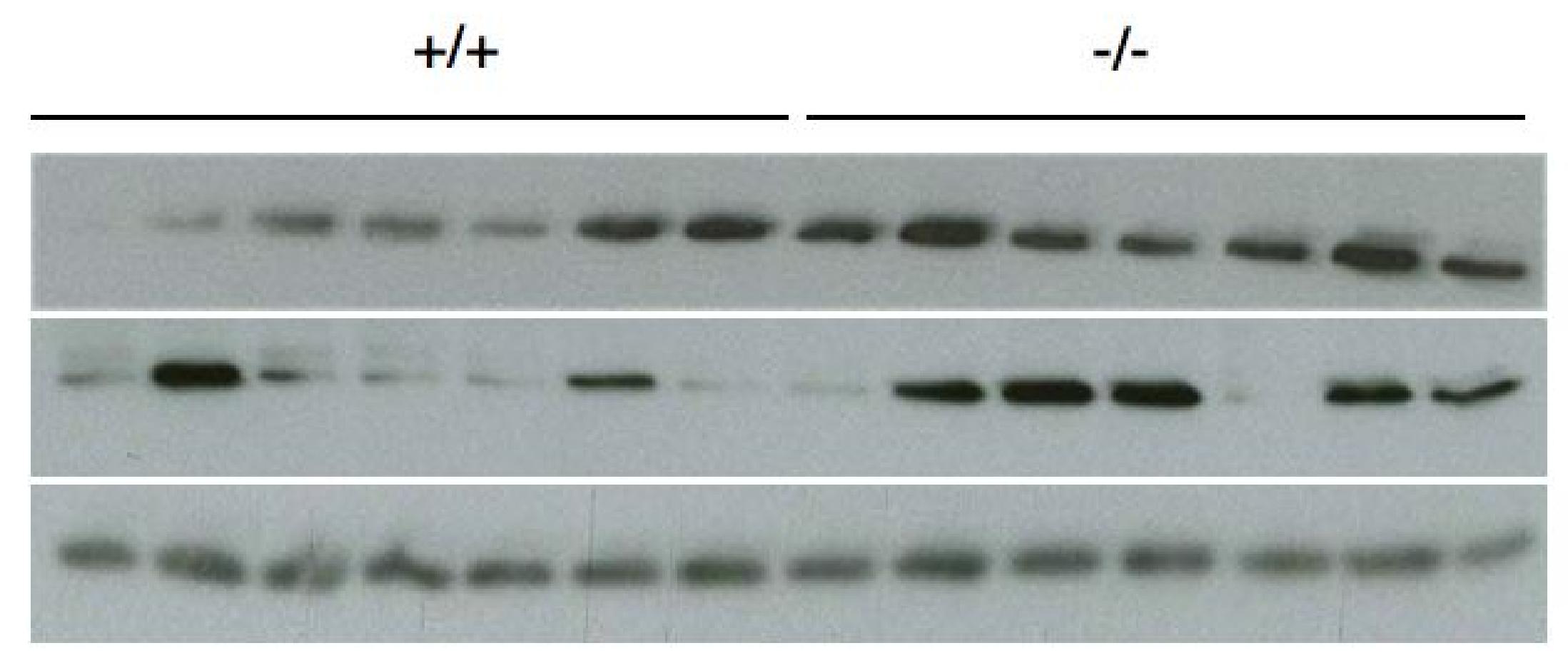

BIP-1

CHOP

ACTIN

$\mathbf{F}$
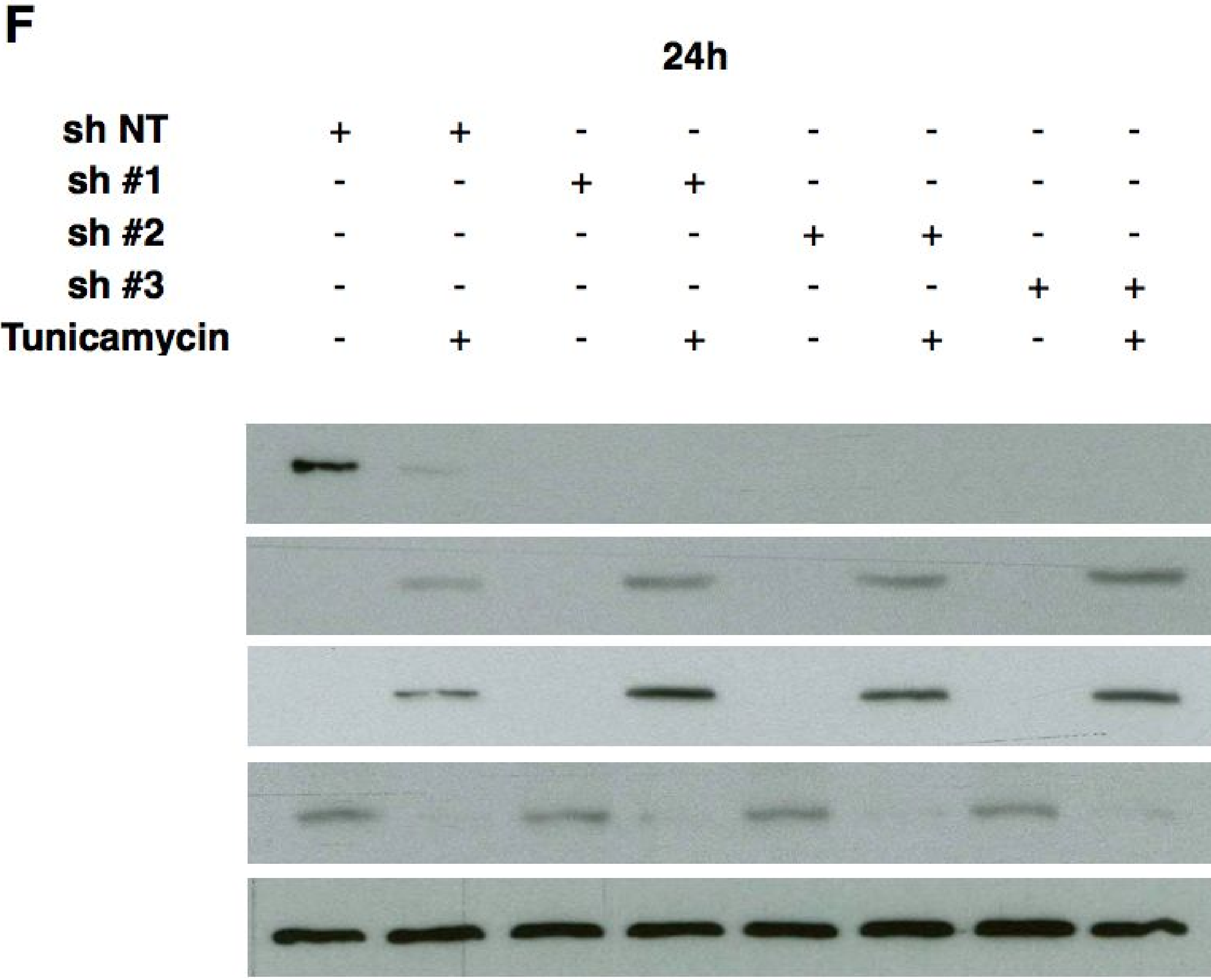

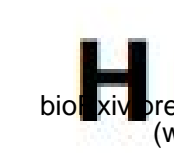

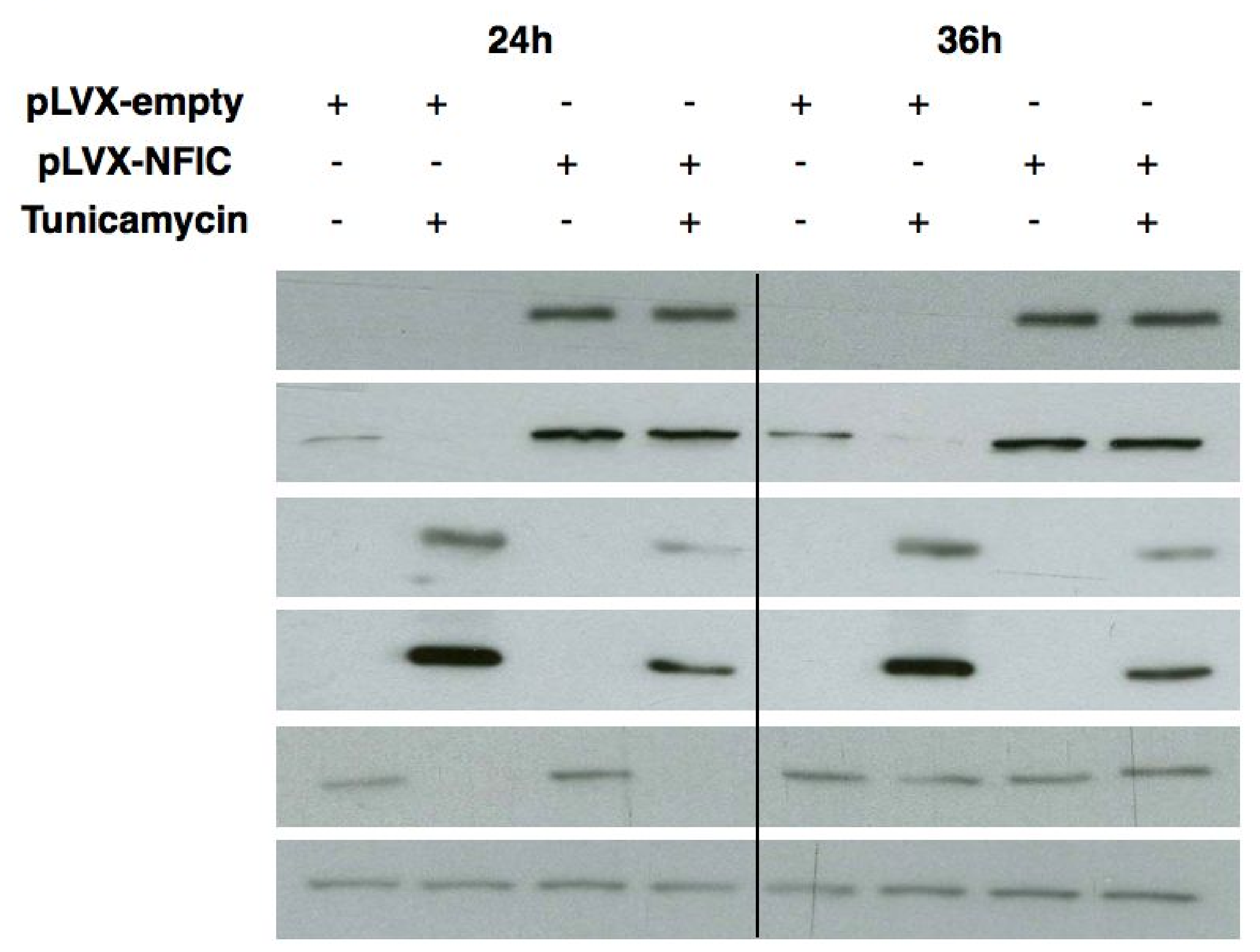

B
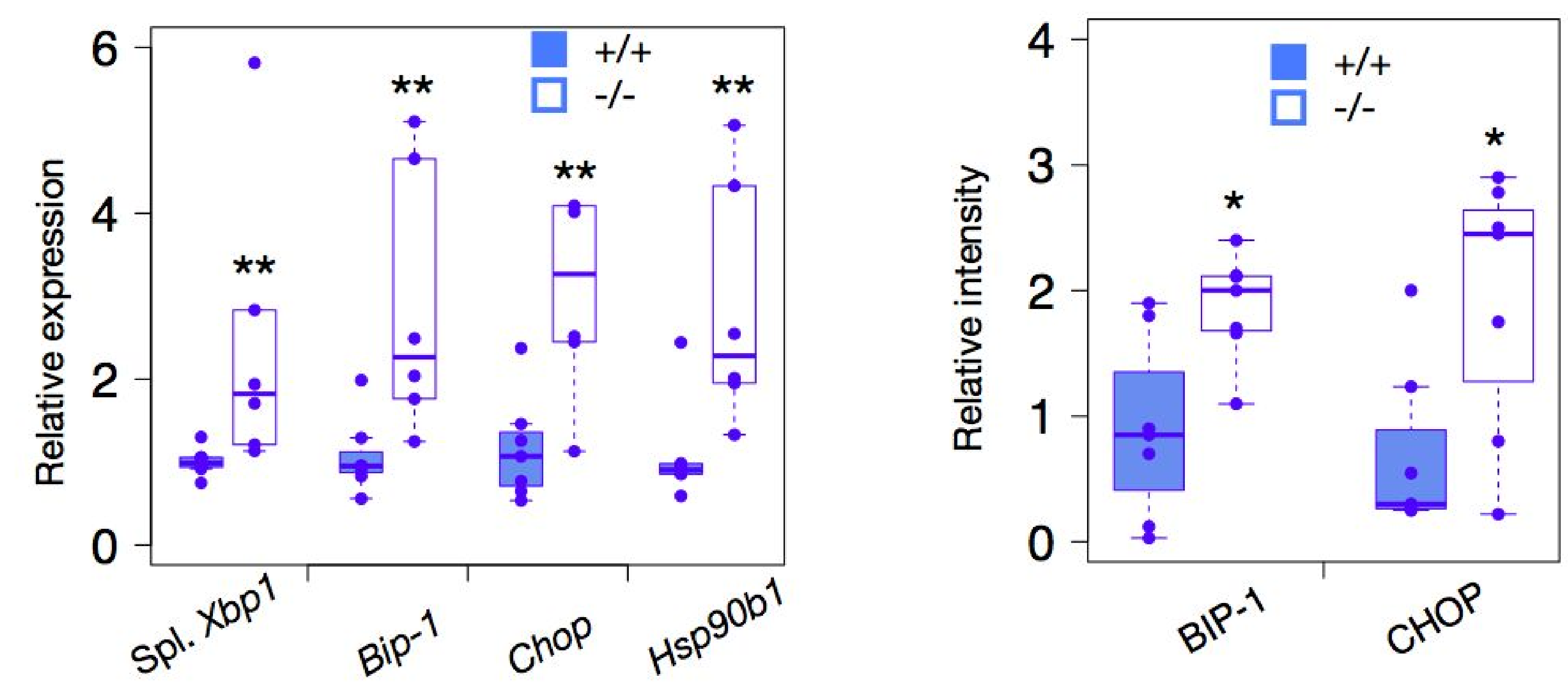

E
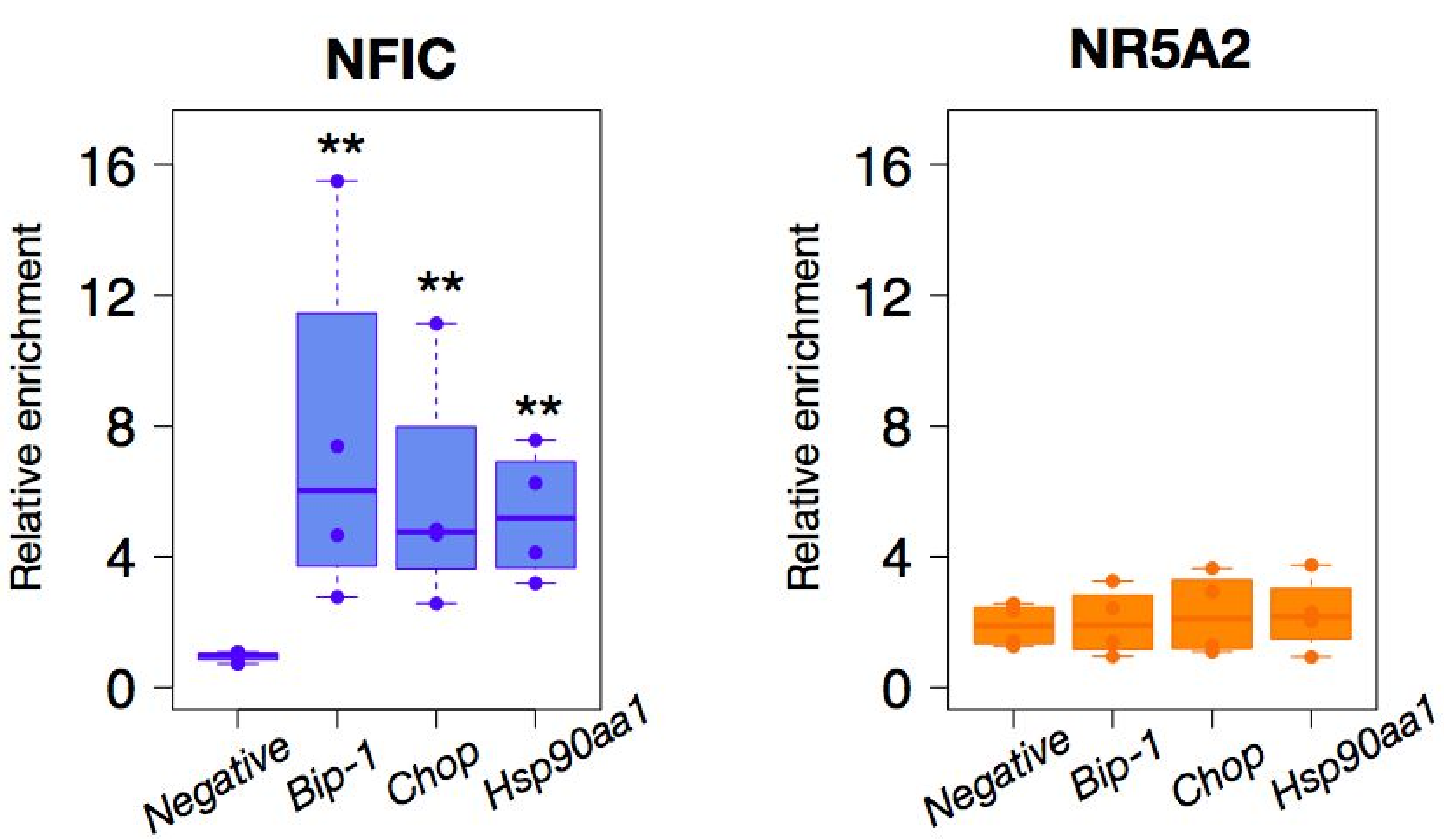

NFIC

BIP-1

CHOP

NR5A2

VINCULIN

HA-tag

NFIC

BIP-1

CHOP

NR5A2

VINCULIN
G

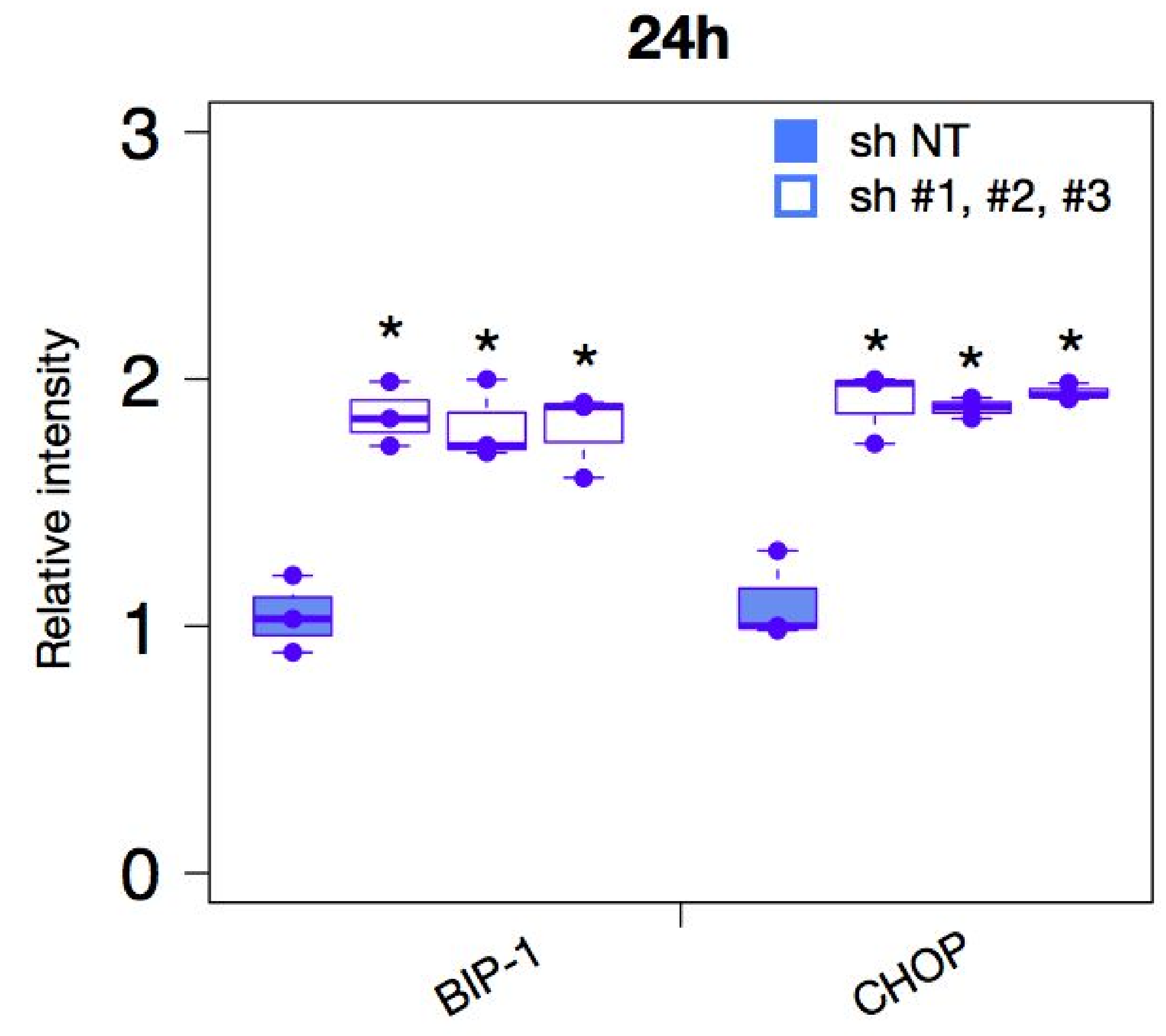

I

24h

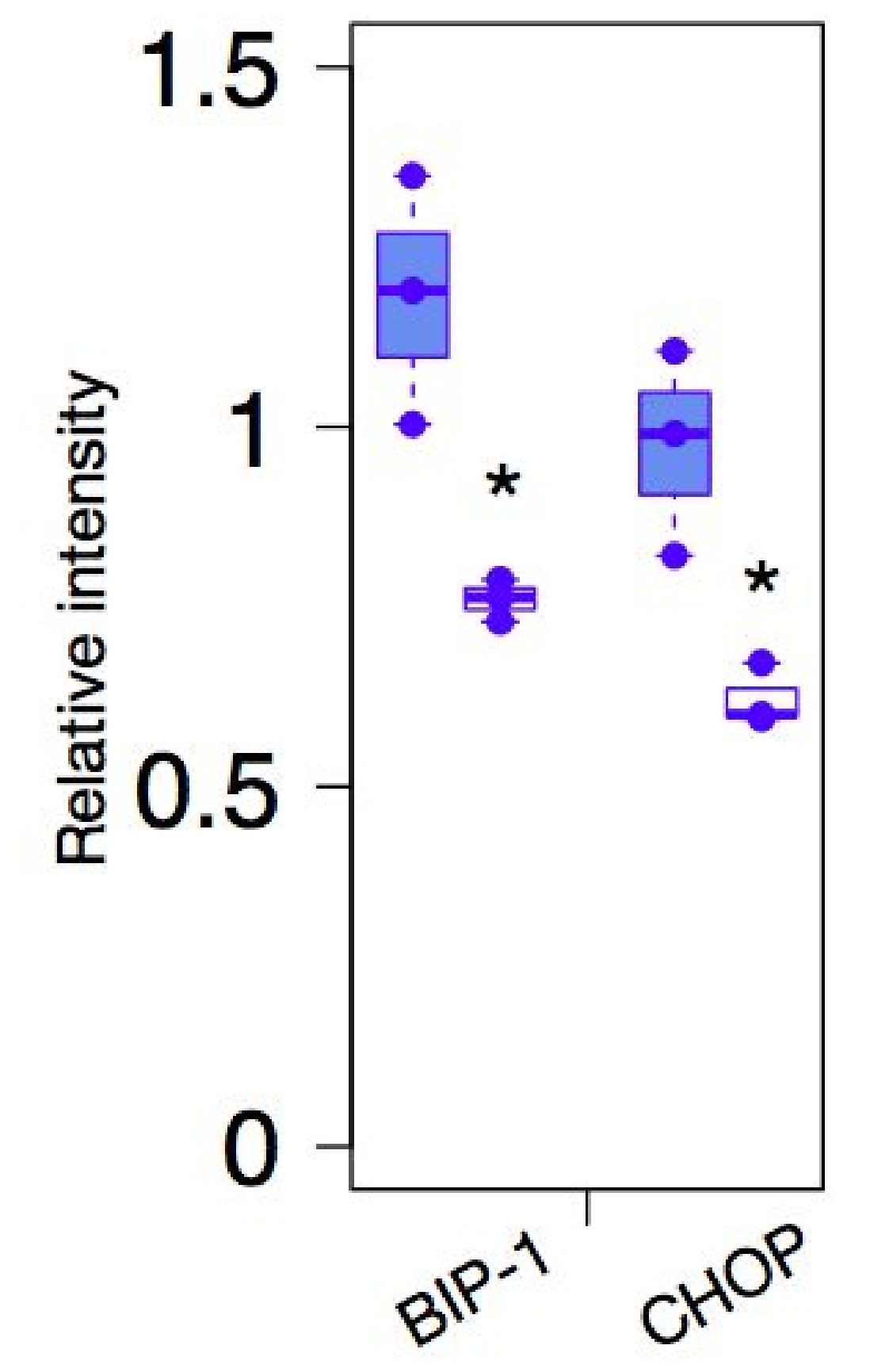

36h

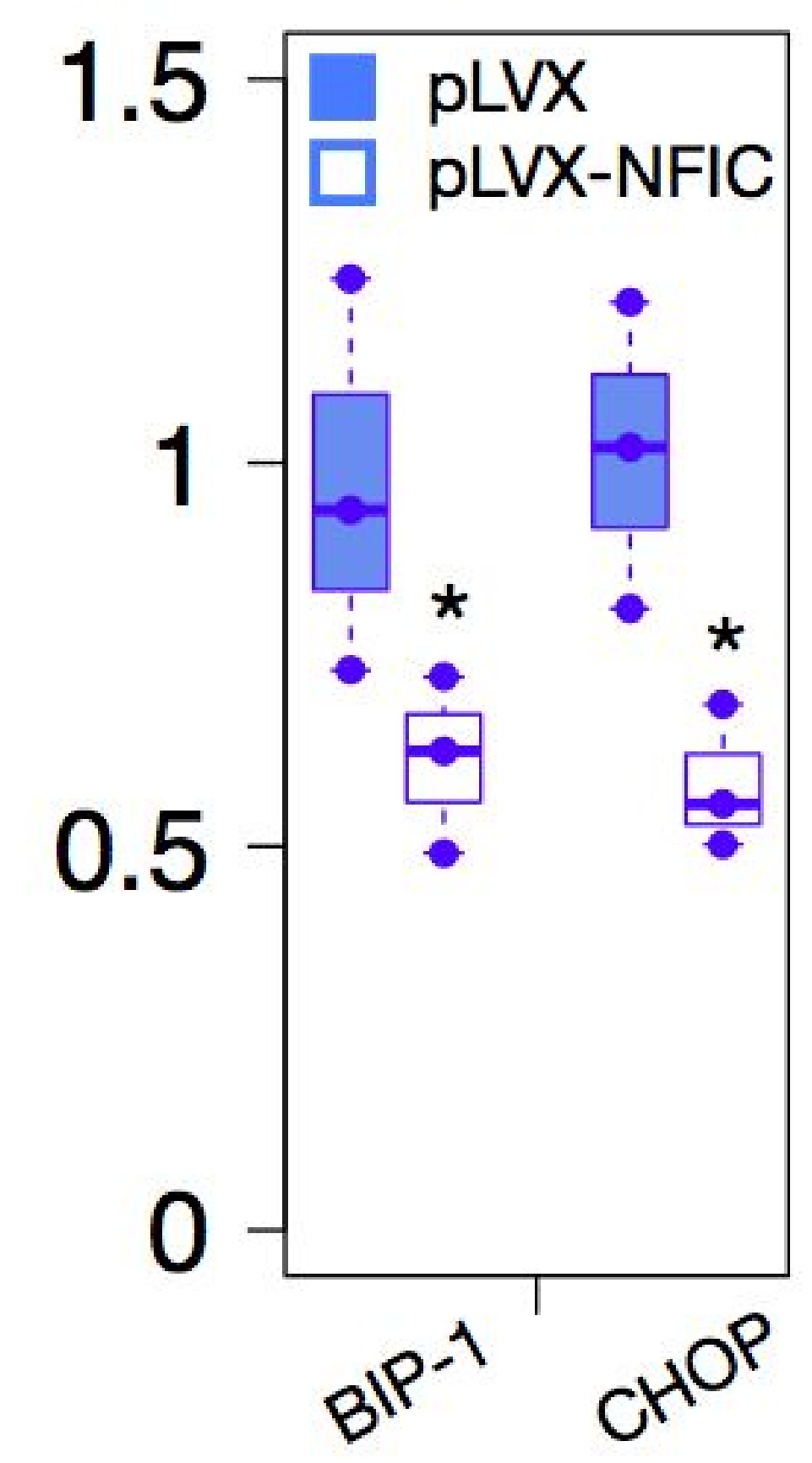




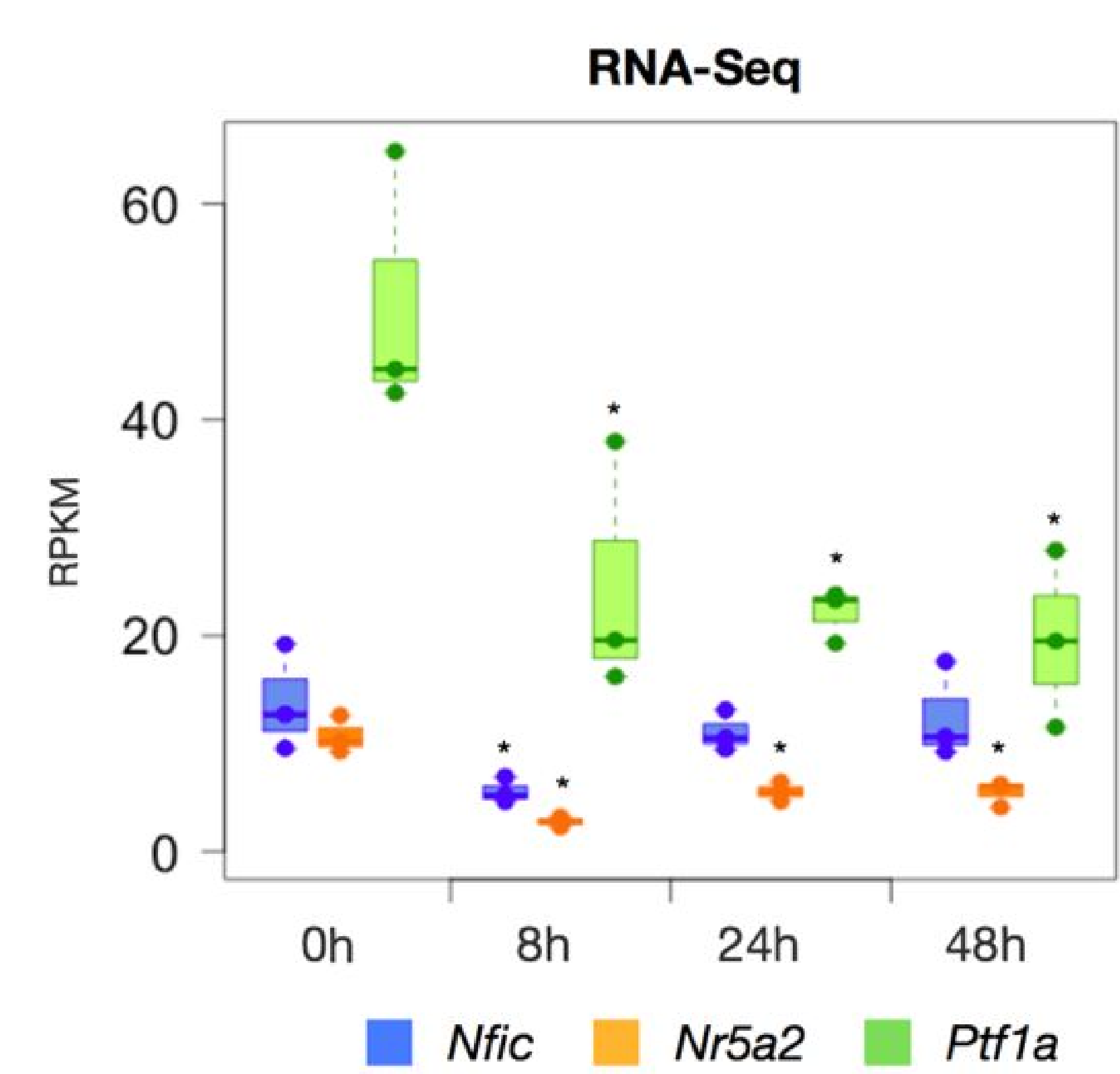

B

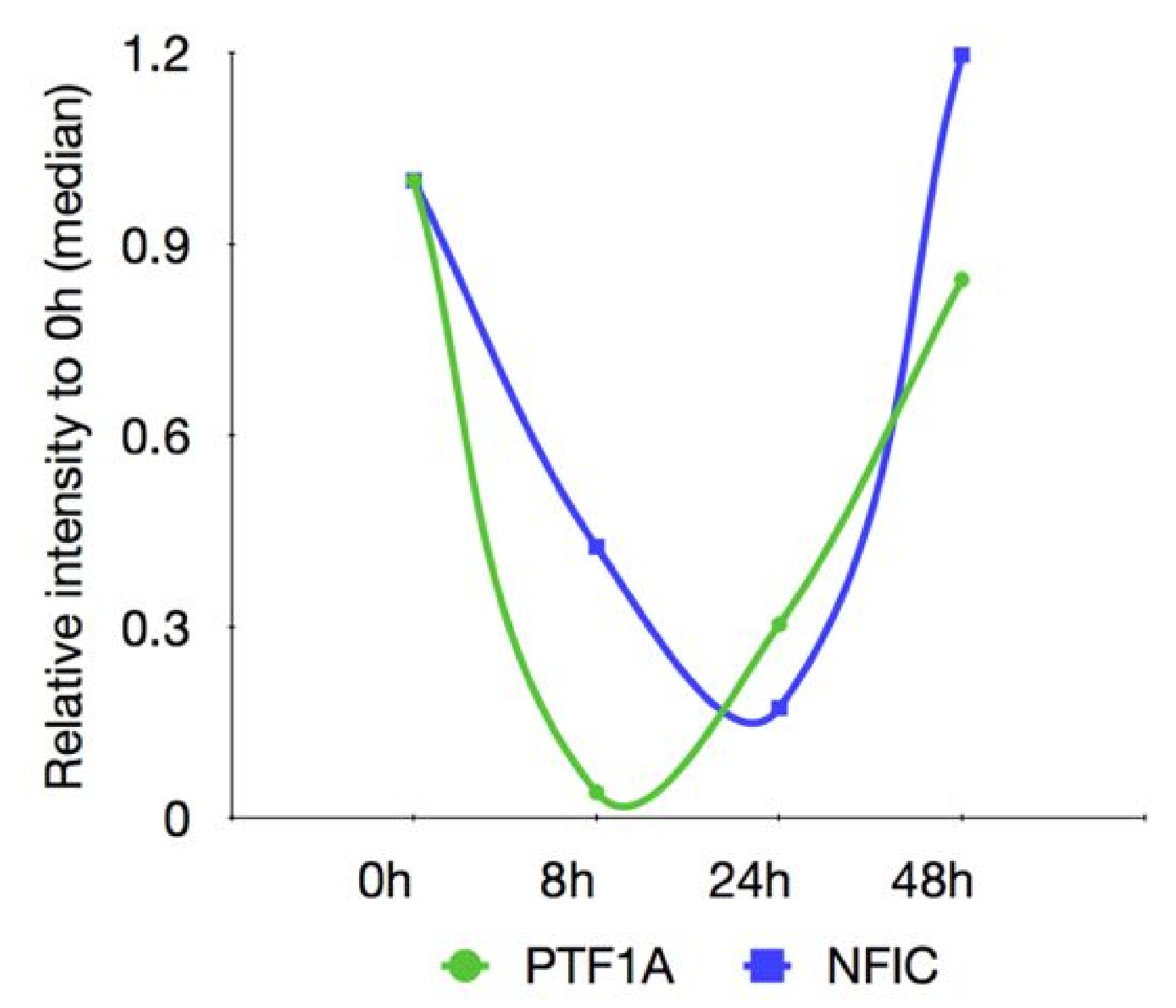

D
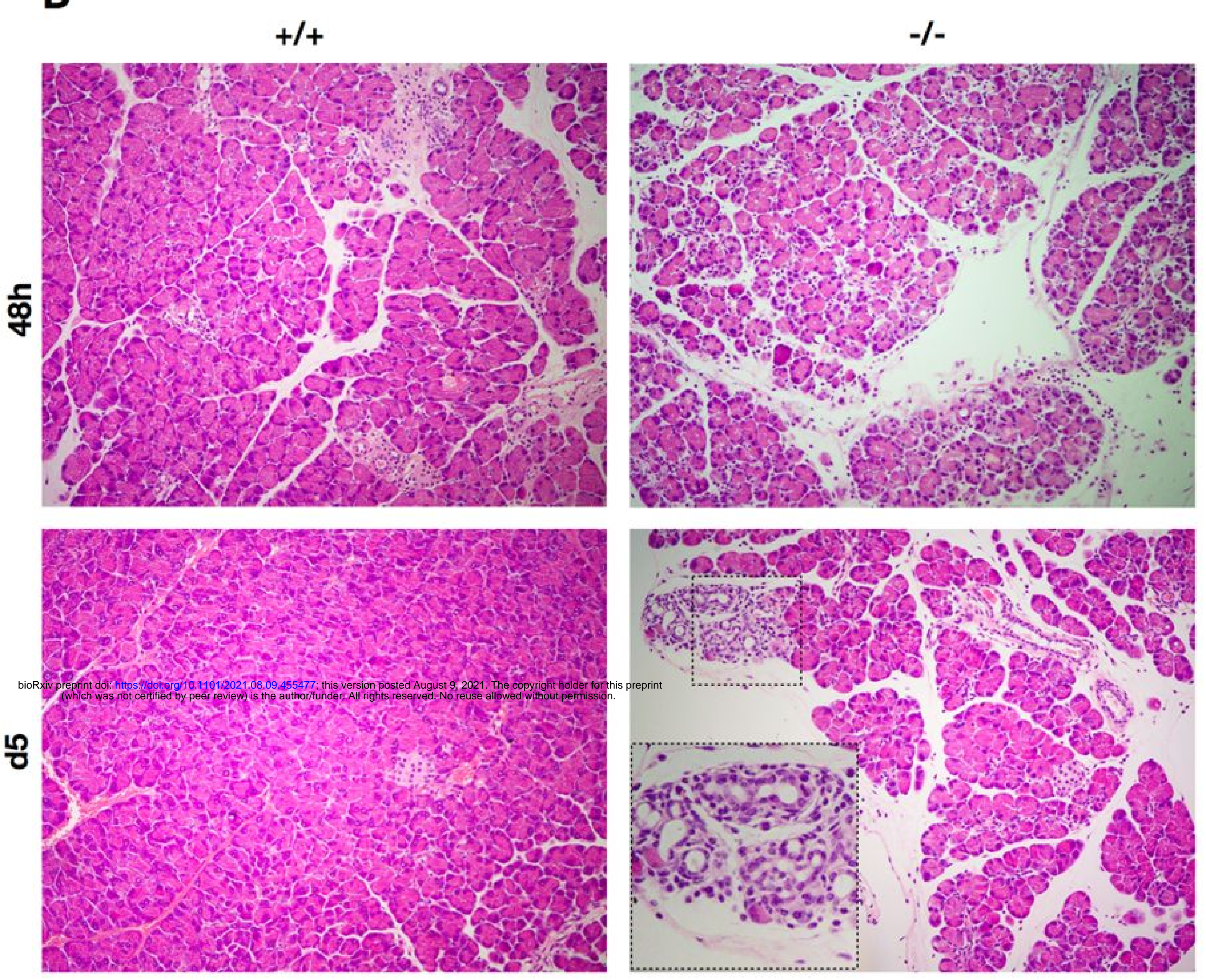

E

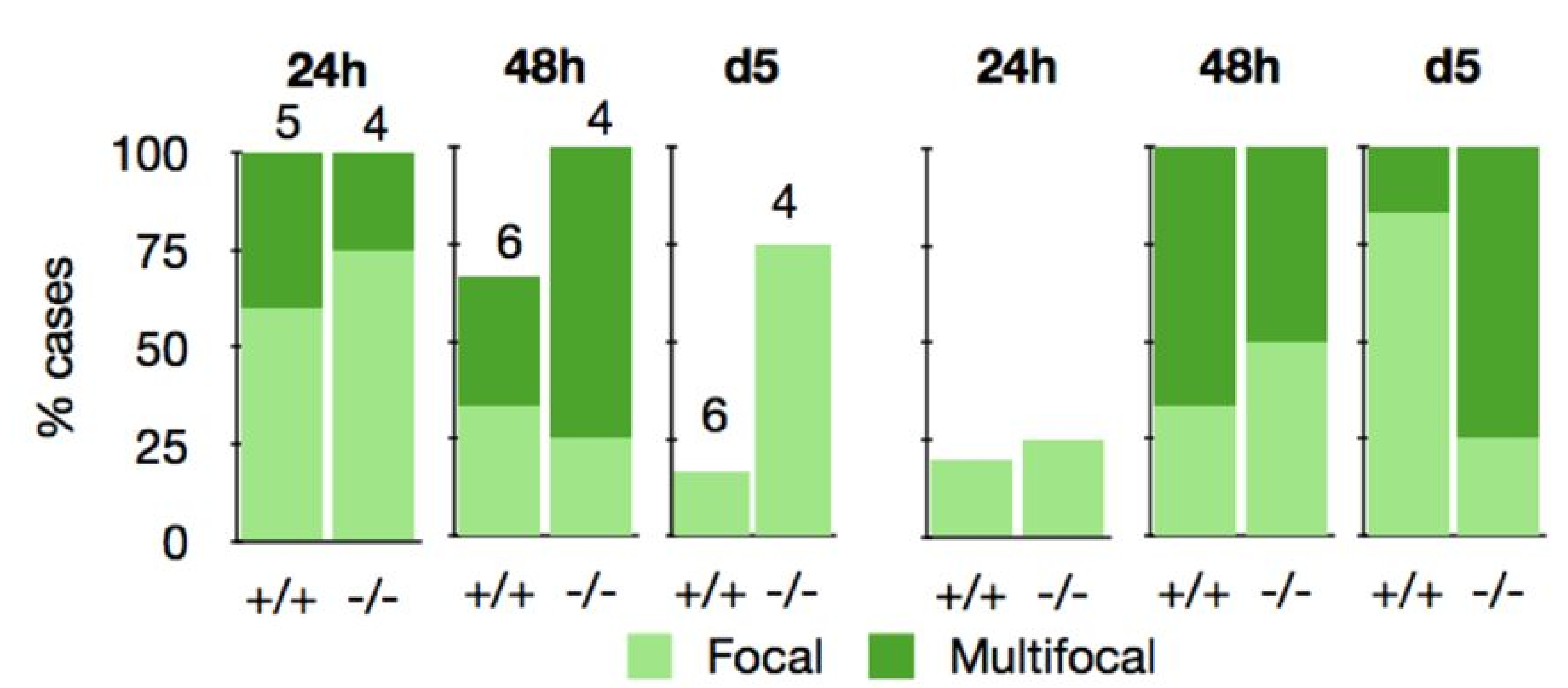

C

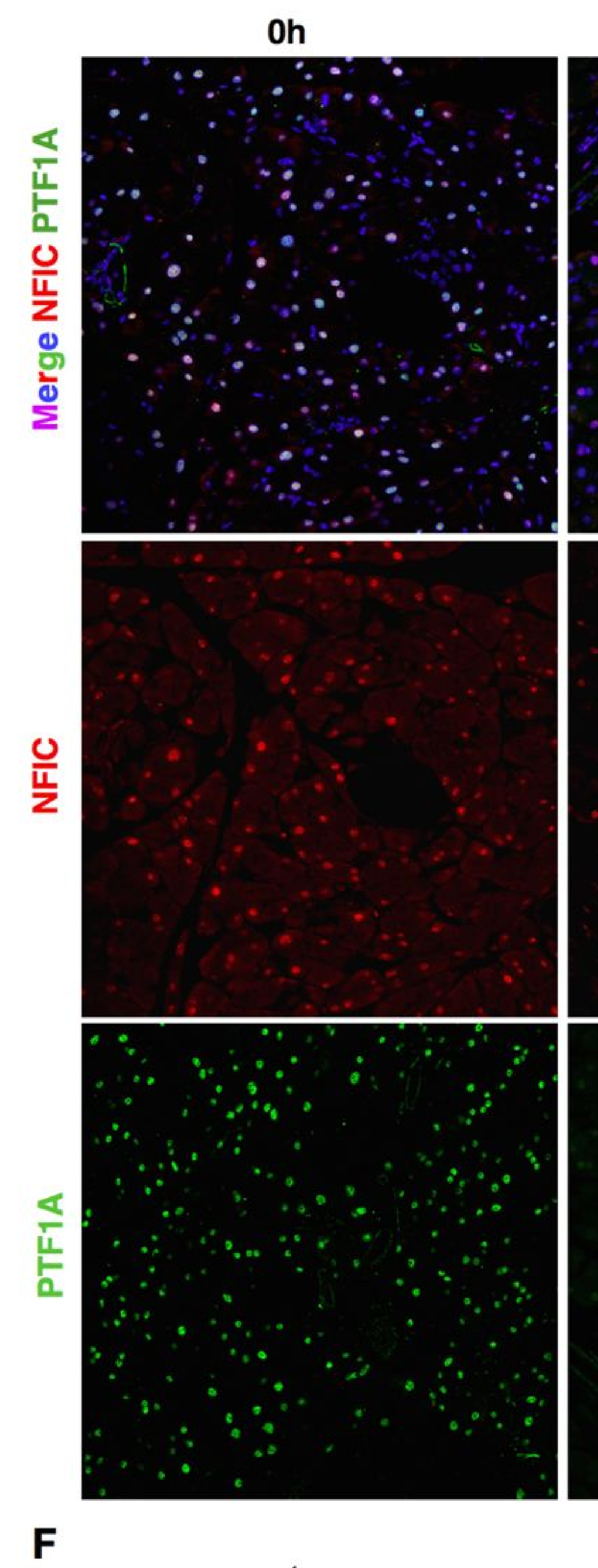

$8 h$

24h

$48 h$

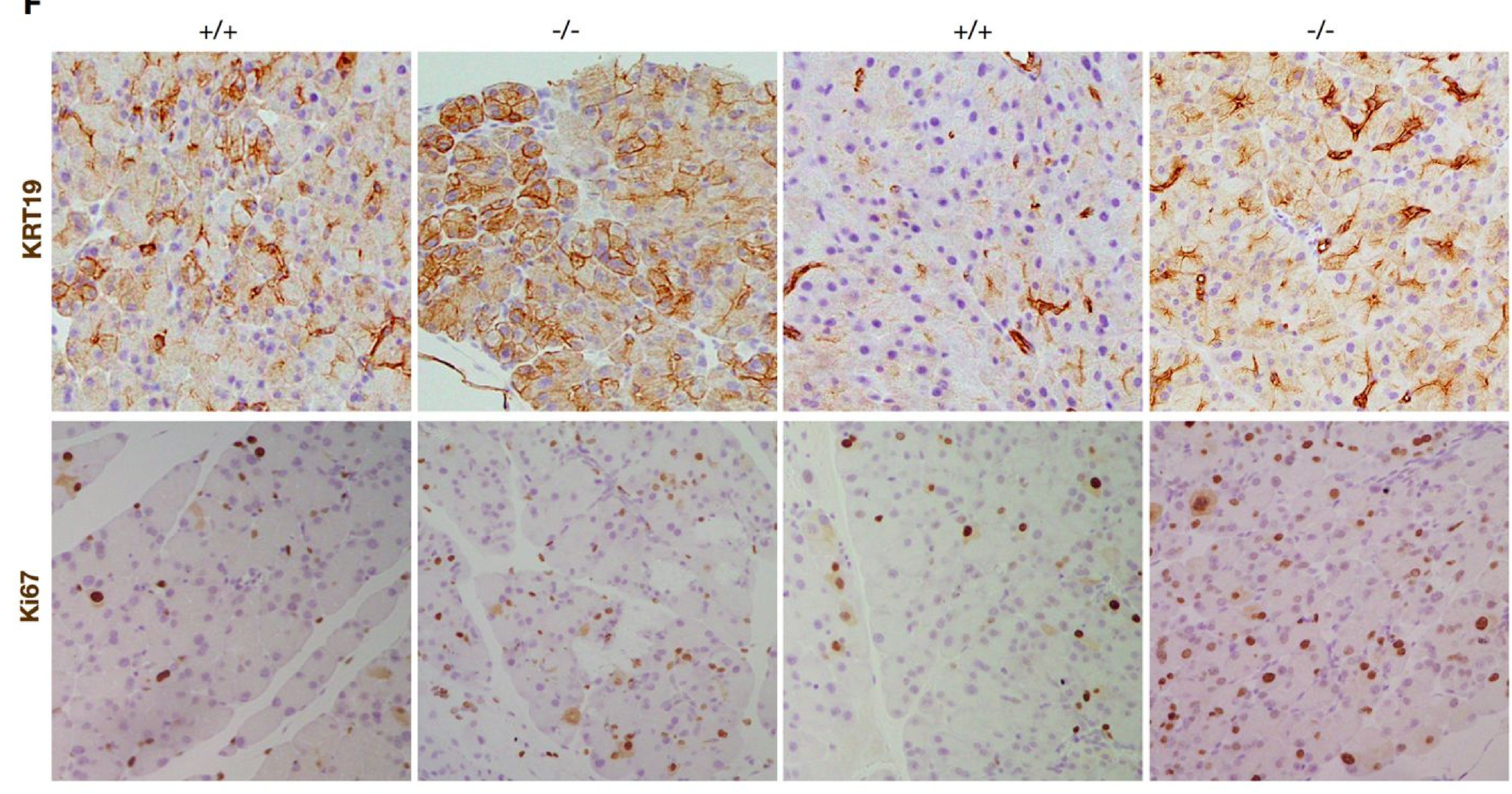

48h

G

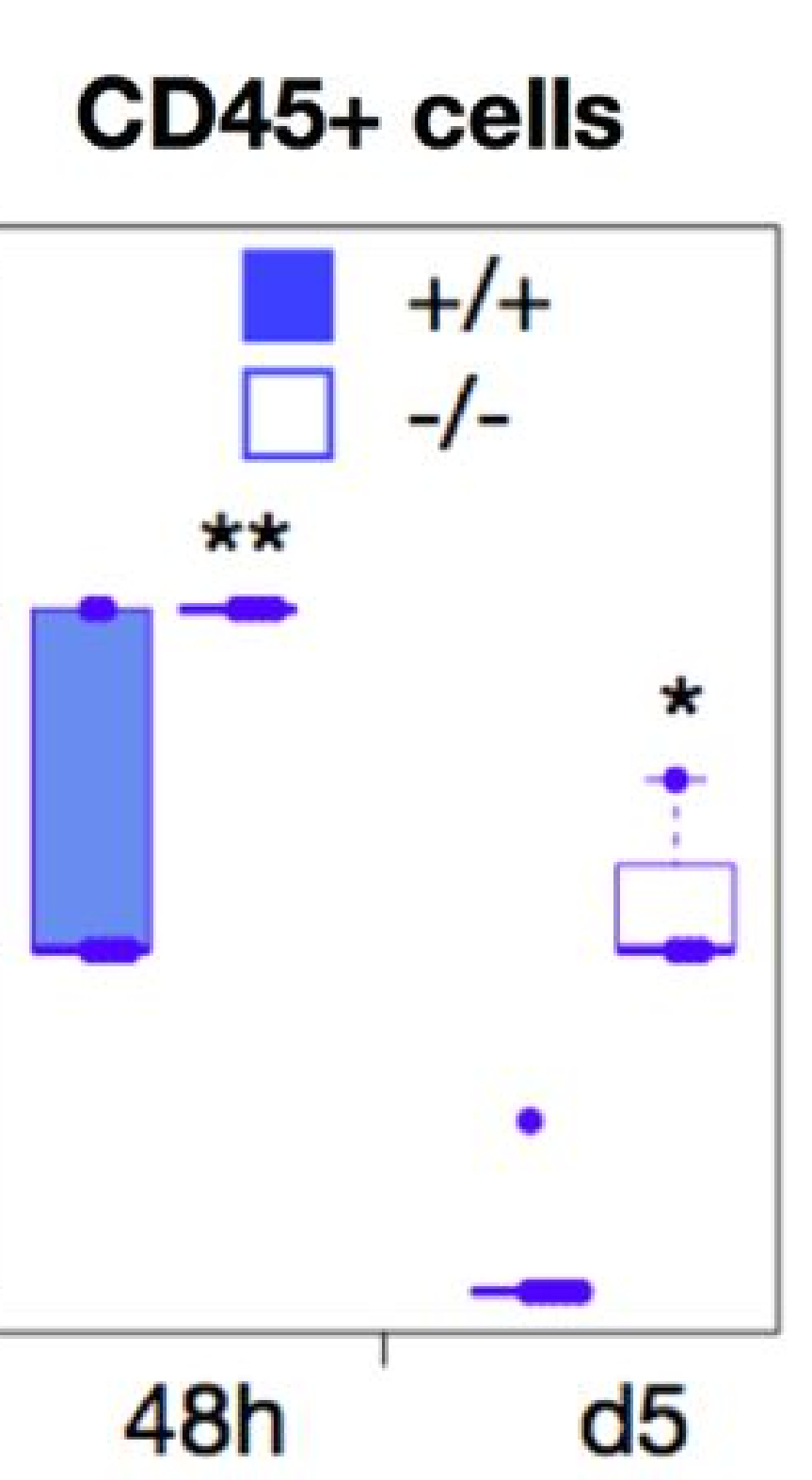

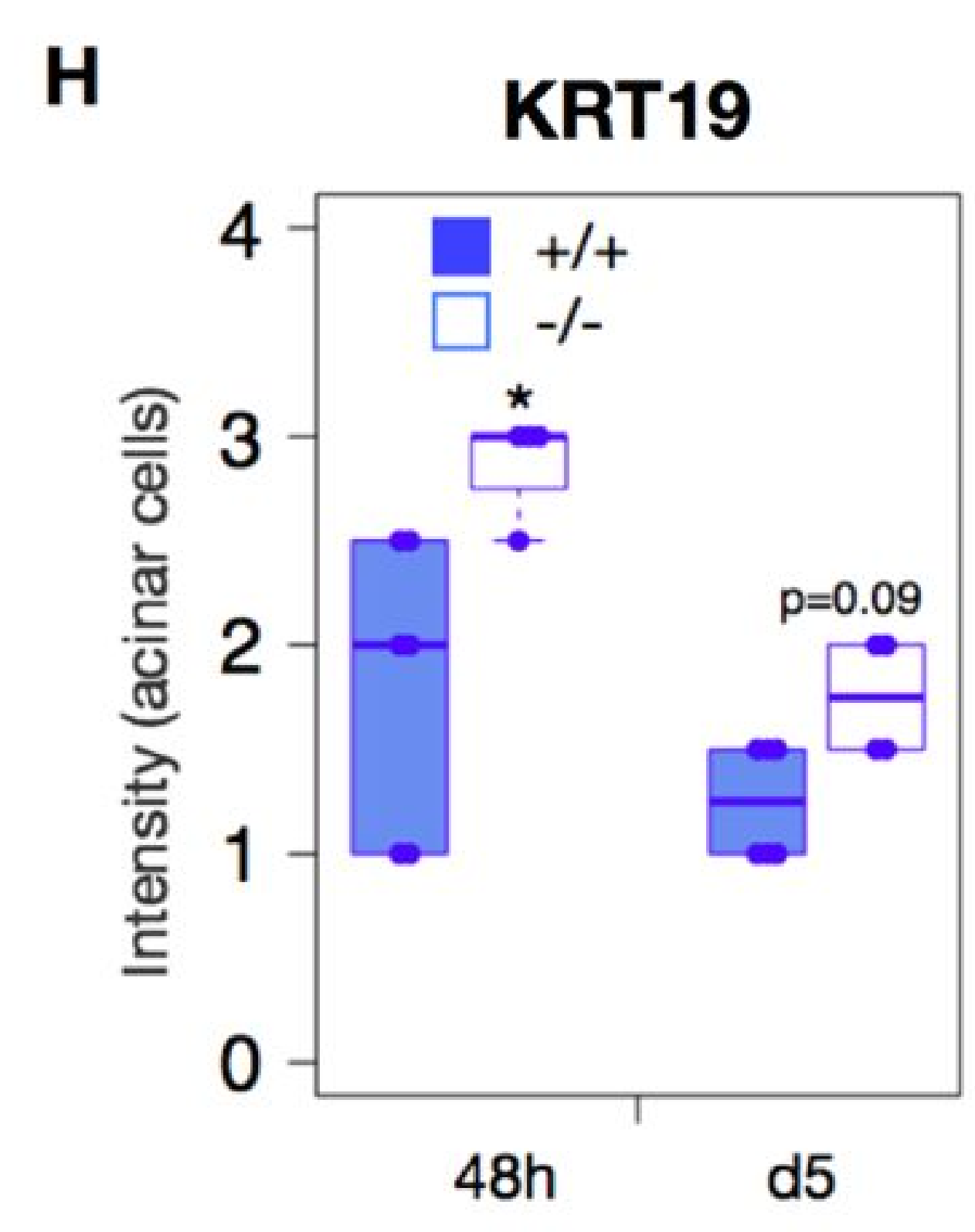

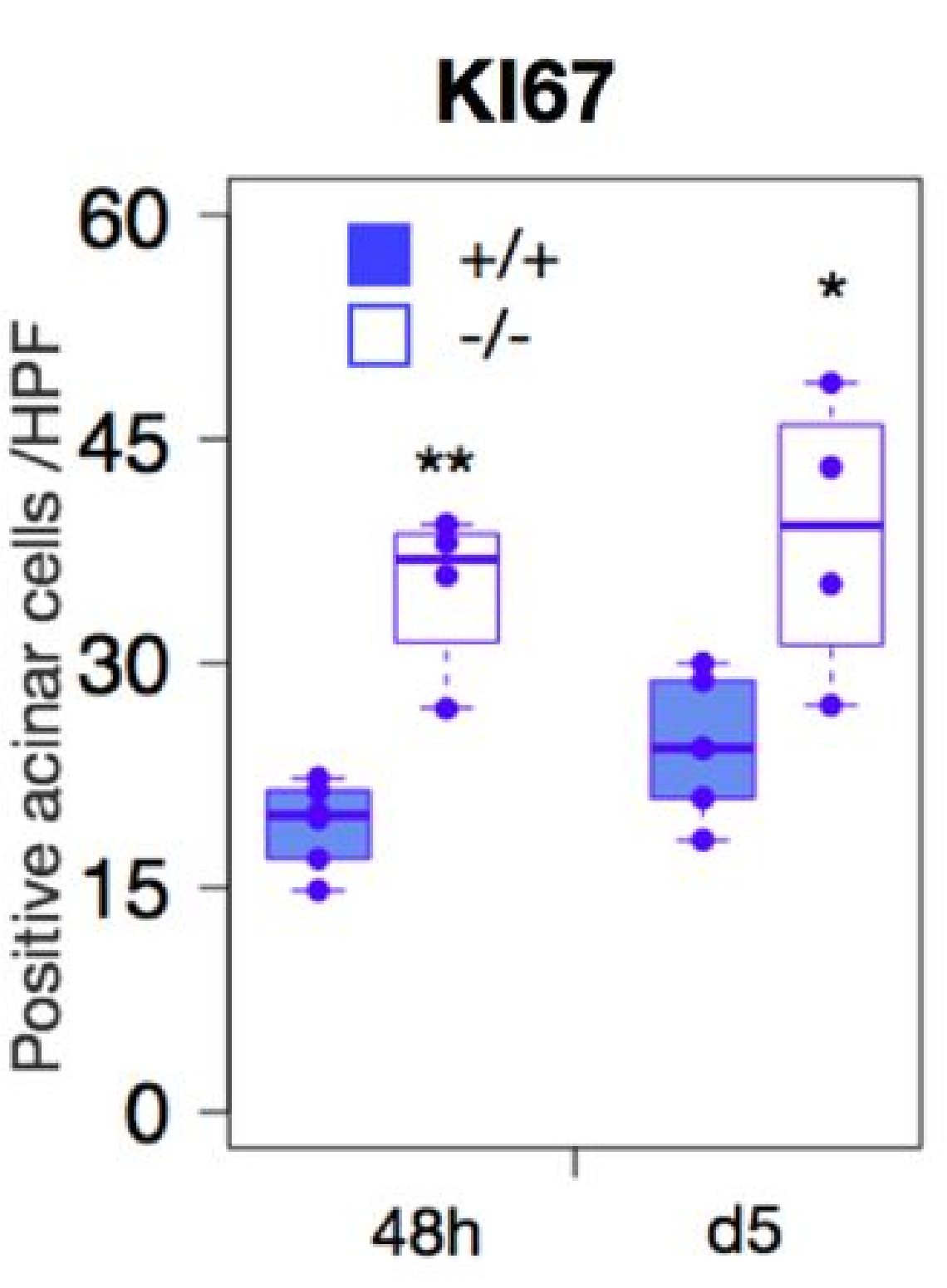



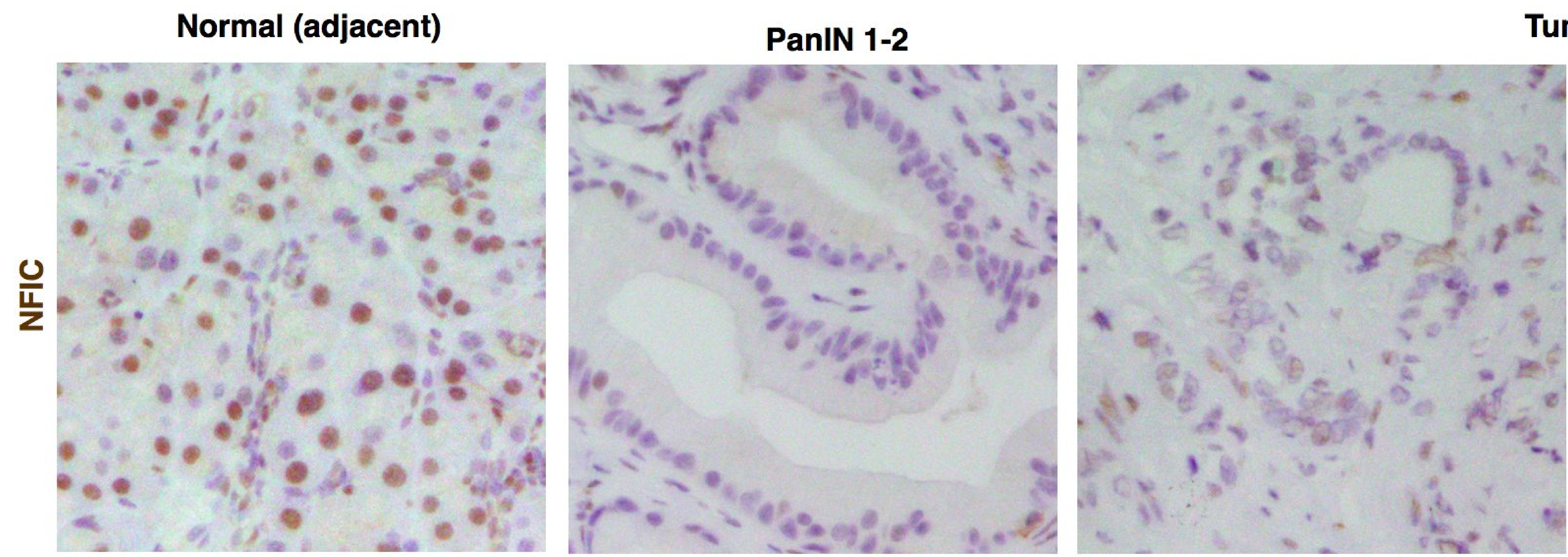

Tumour

B

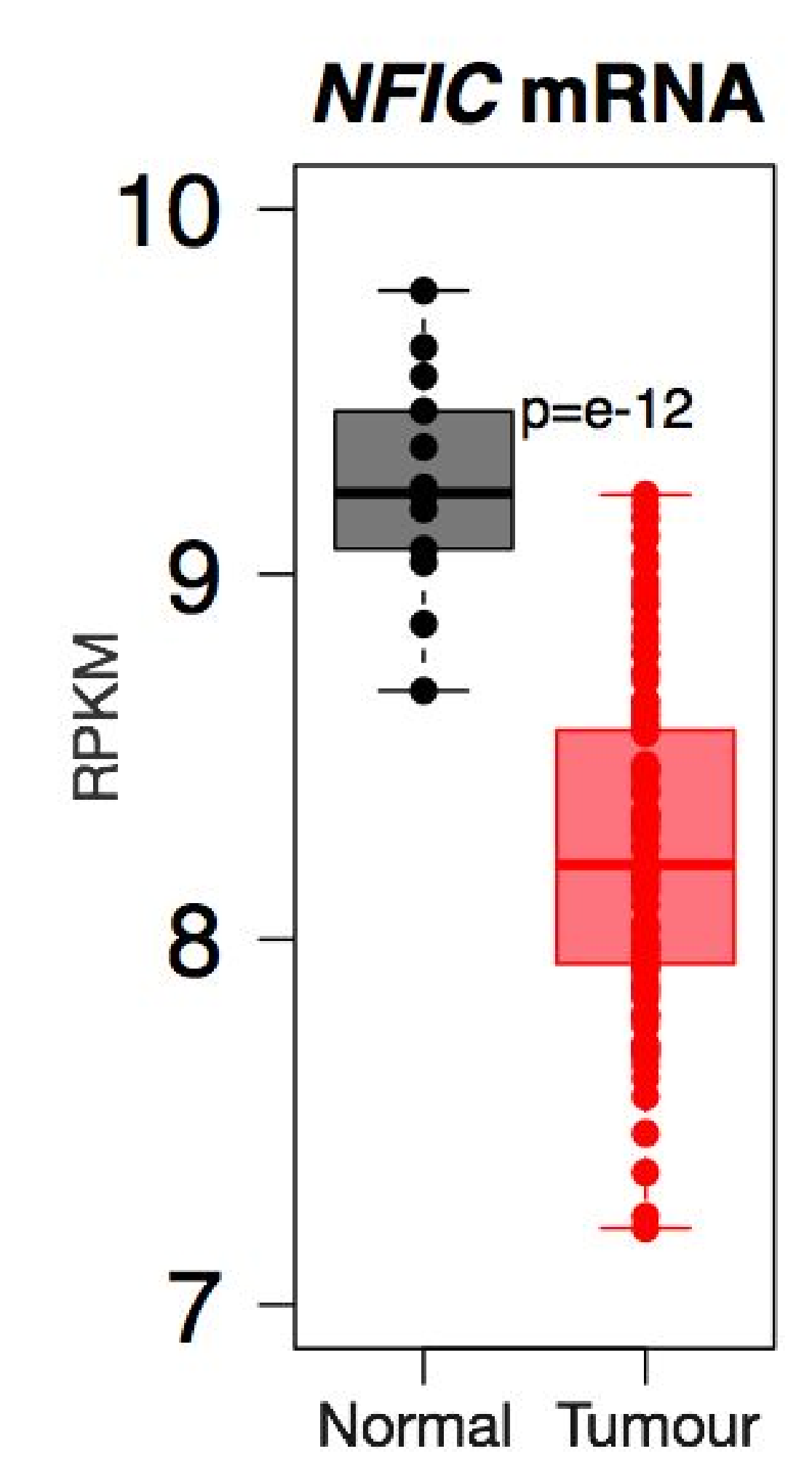

C

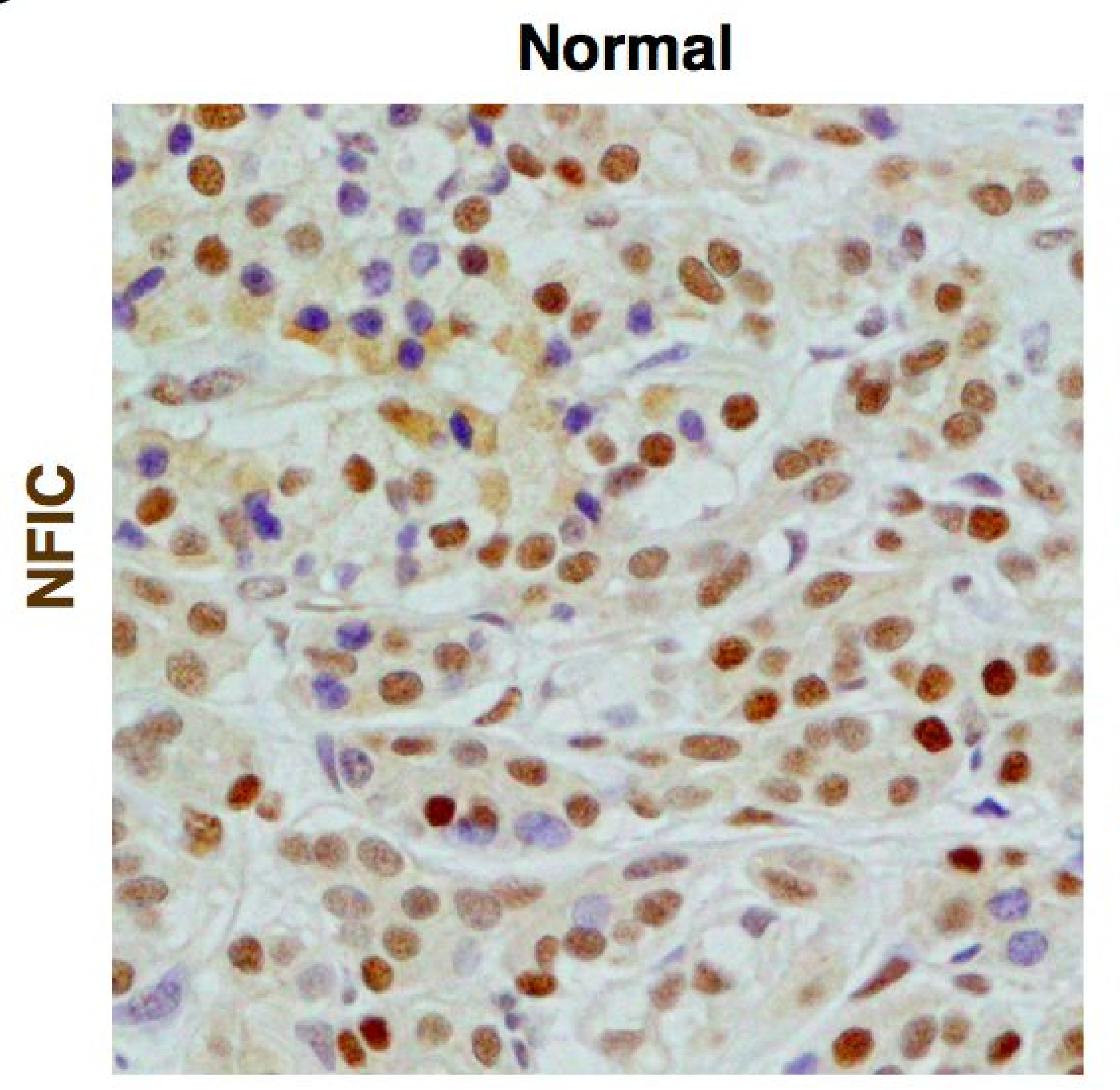

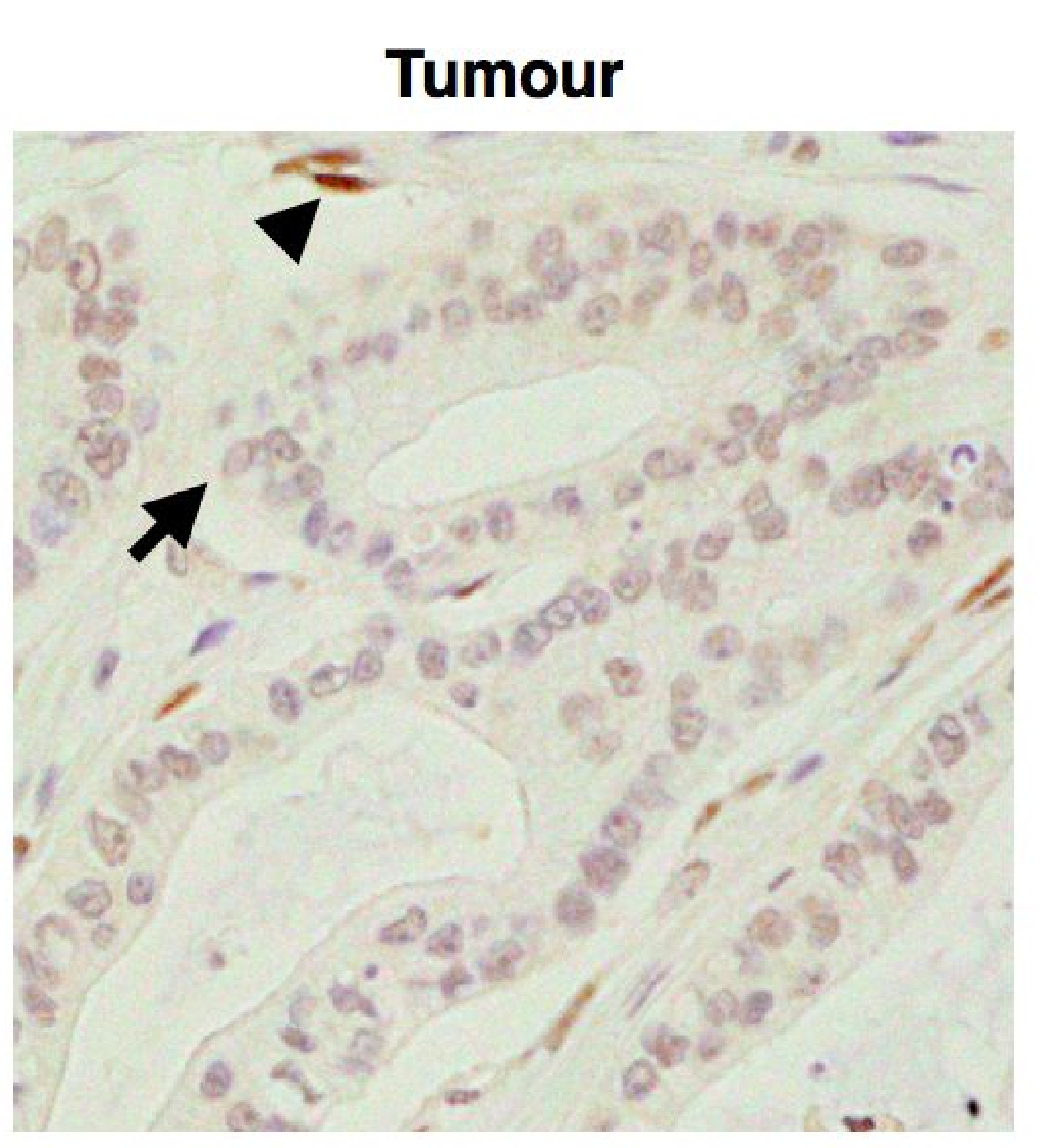

D
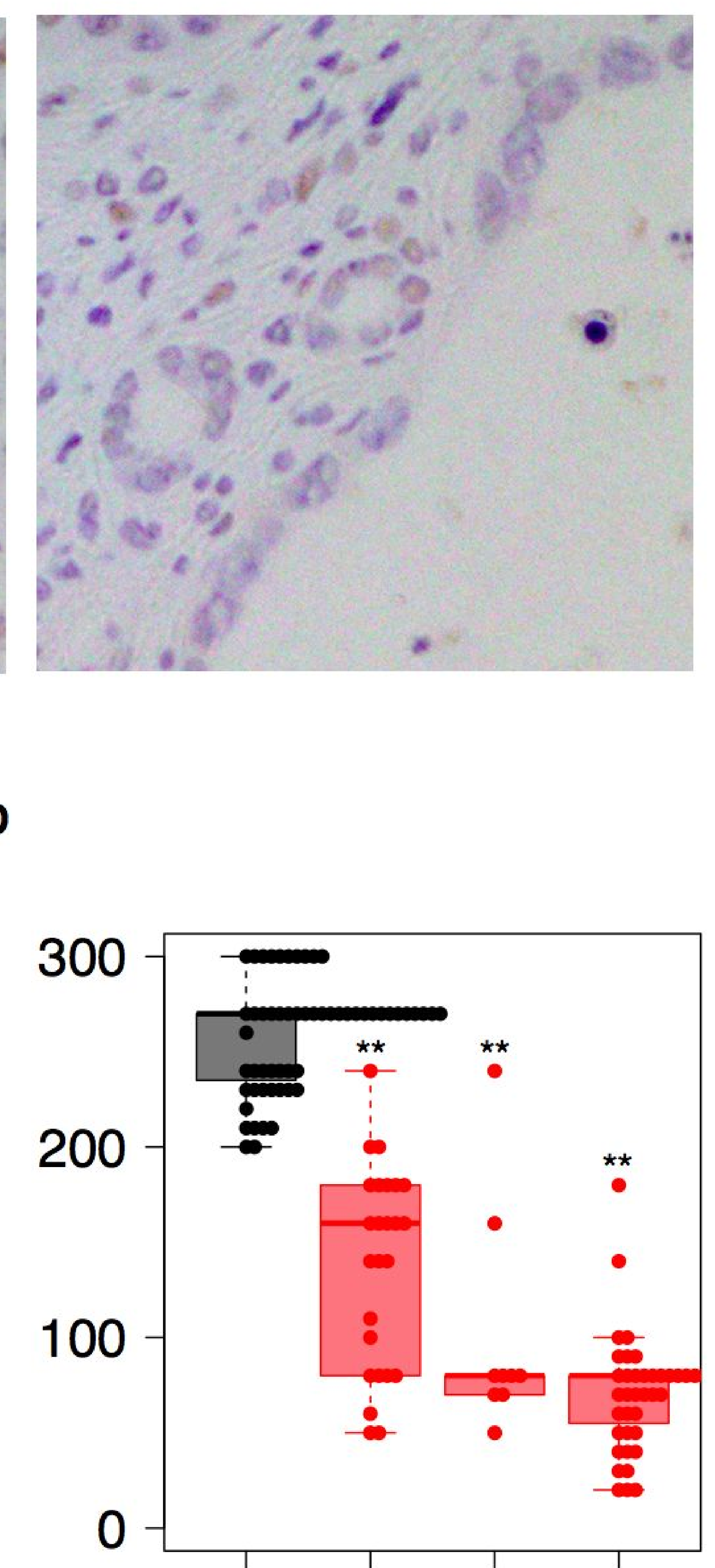

E

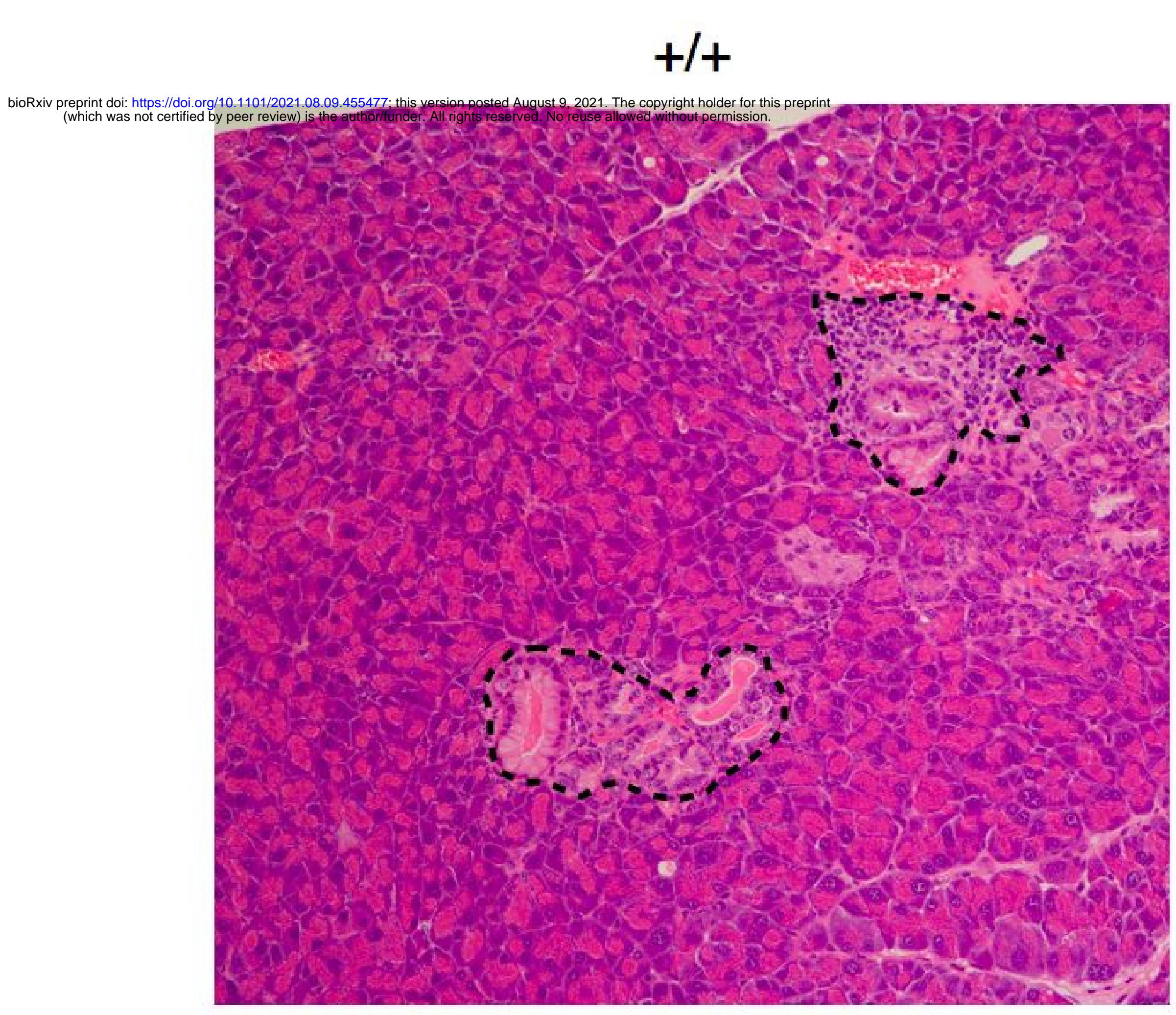

Kras ${ }^{\mathrm{G} 12 \mathrm{~V}}$

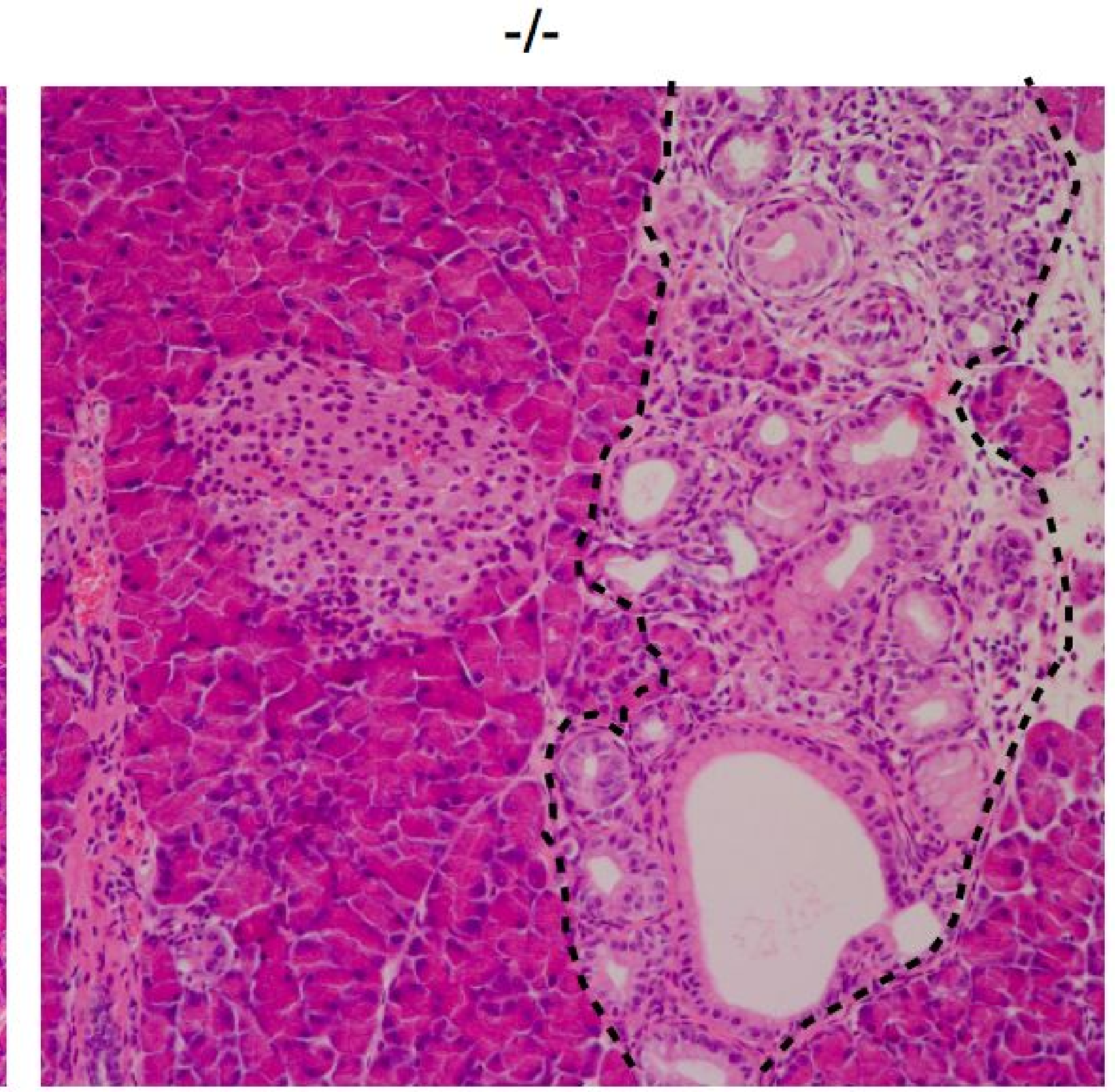

F

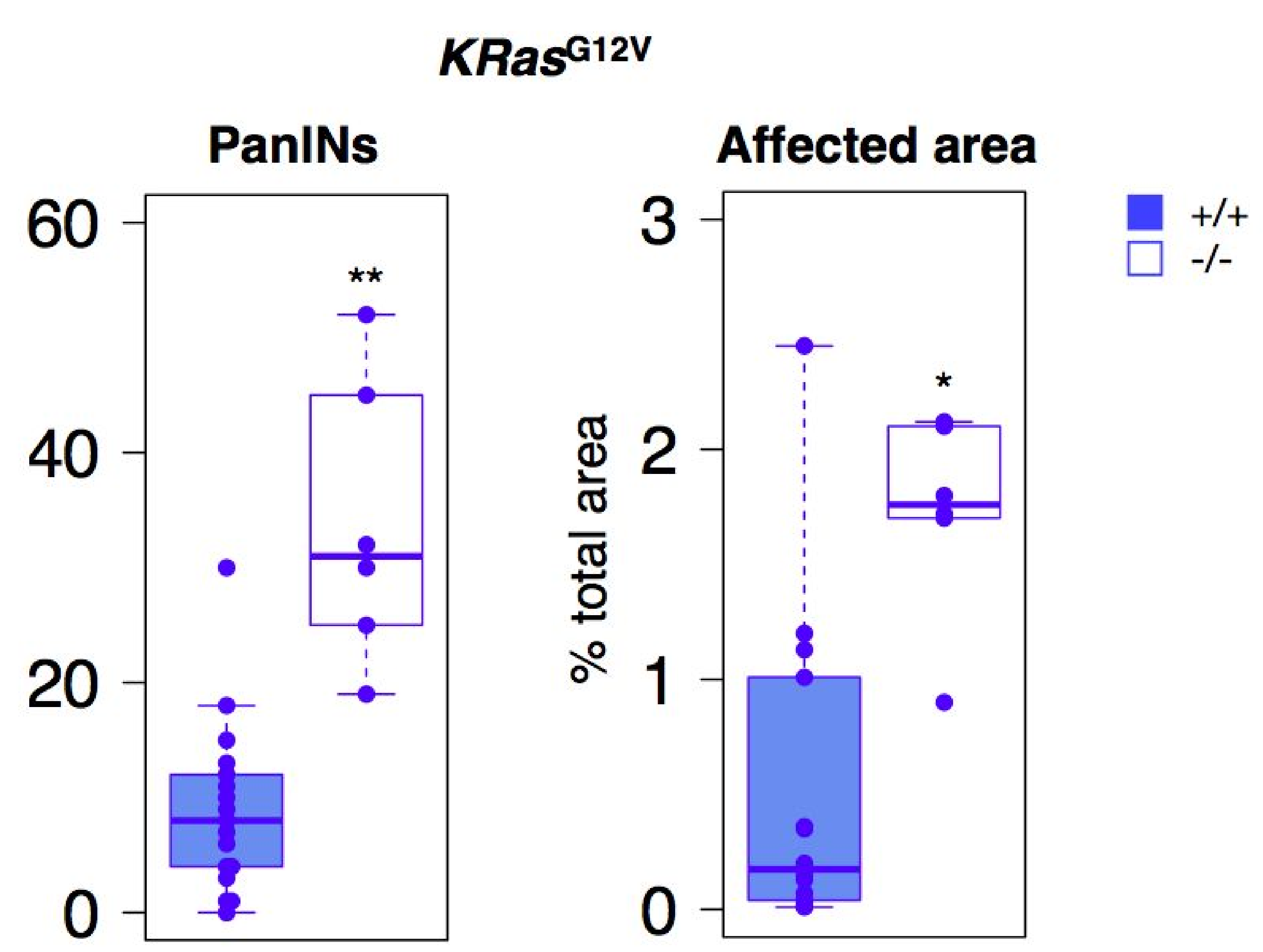

